\title{
3,4-Bisthiolated pyrroles: concise construction and their electronic properties
}

\author{
Jun Tian, ${ }^{\text {a }}$ Kai Feng, ${ }^{a}$ Kangning Yuan, ${ }^{a}$ Xing Li, ${ }^{a}$ Hong-Hong Chang ${ }^{a, c}$ and Wen-Chao Gao*a,b,c \\ ${ }^{a}$ College of Biomedical Engineering, Taiyuan University of Technology, Taiyuan 030024, China. \\ ${ }^{b}$ School of Chemistry and Molecular Engineering, East China Normal University, Shanghai, 200062, China \\ ${ }^{c}$ Shanxi Tihondan Pharmaceutical Technology Co., Ltd. Jinzhong 030600, China. \\ E-mail: gaowenchao@tyut.edu.cn
}

\section{Table of Contents}

Electrochemical data of representative bisthiopyrroles...................S2

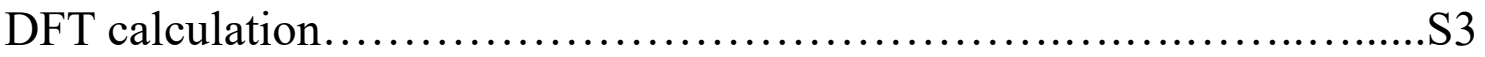

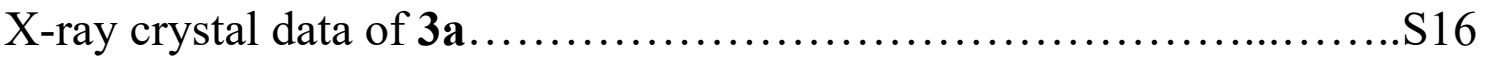

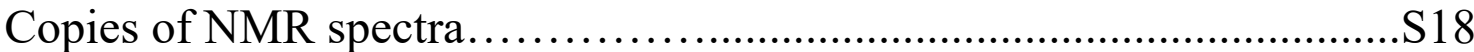

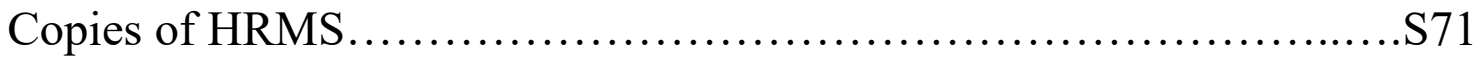




\section{Electrochemical Data of representative bisthiopyrroles}

The electrochemical properties of 3,4-bisthio pyrroles were investigated by cyclic voltammetry (CV) and differential-pulse voltammetry (DPV) experiments in $\mathrm{CH}_{3} \mathrm{CN}$ solution containing $0.1 \mathrm{M}$ tetra-n-butylammonium tetrafluoroborate as the supporting electrolyte.

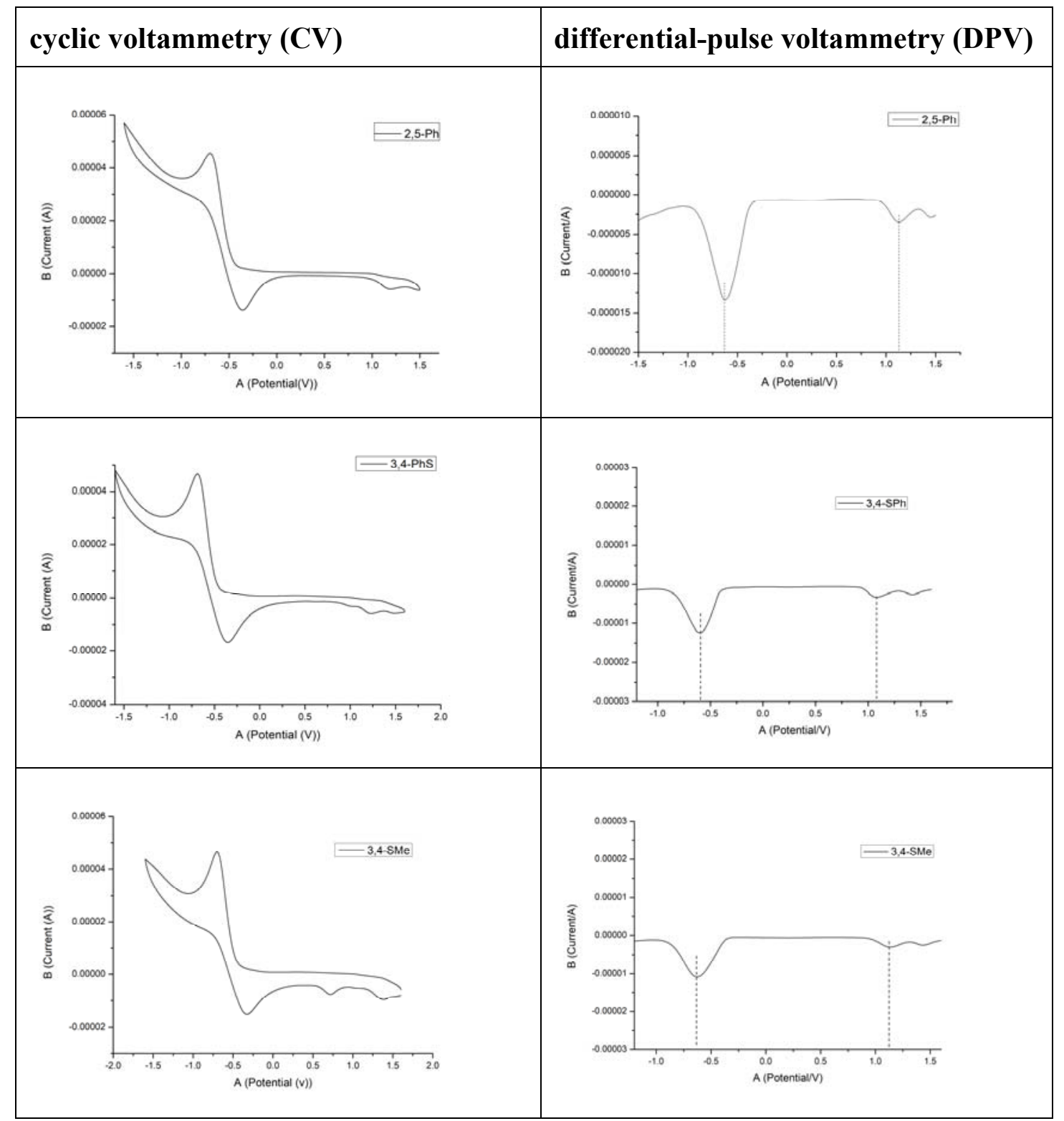




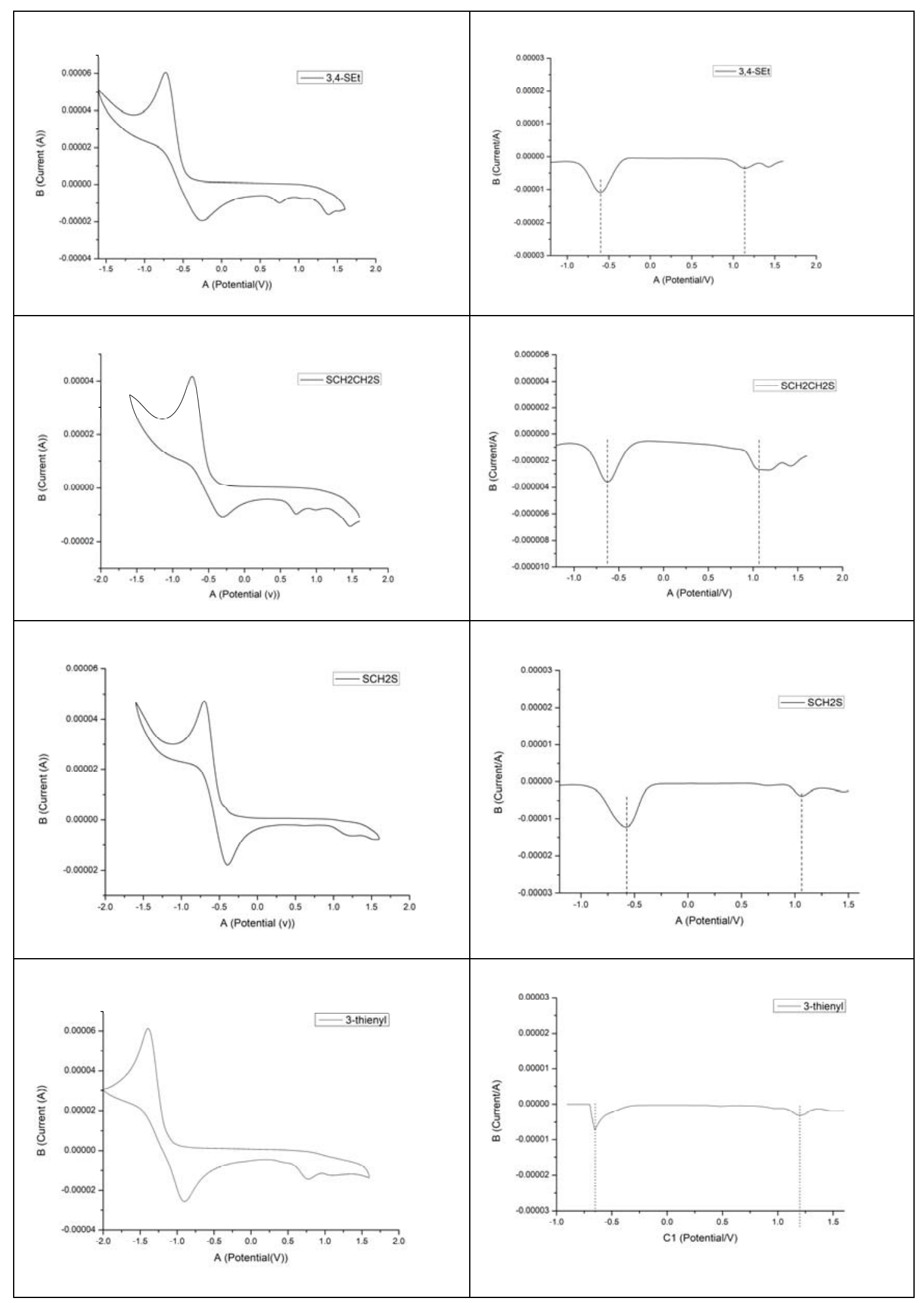




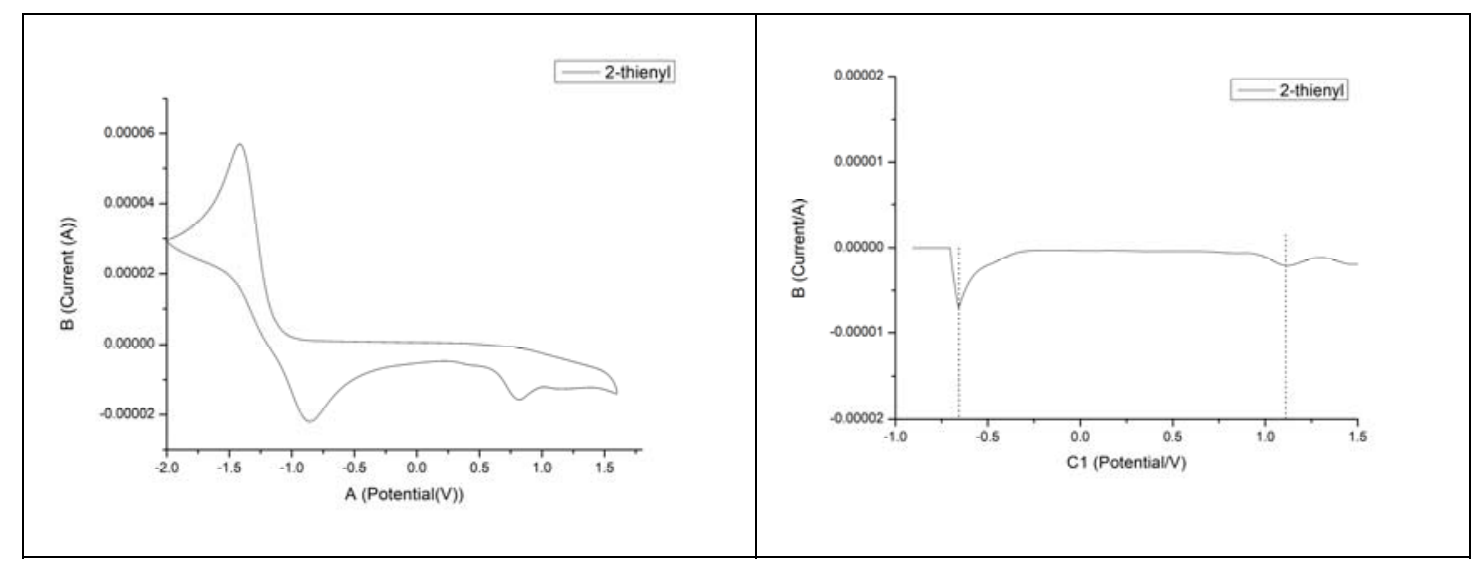

\section{DFT Calculation}

All calculations were performed using the Gaussian 16 A.03 program package. Ground state geometries were optimized by DFT calculations at the B3LYP/6-31G** level. By frequency analysis, structures were onfirmed at the local minima of the potential surfaces. Frequency calculations were carried out for them all to confirm that every structure have no imaginary frequencies indicating that they are true minima. Total energies and thermal corrections to Gibbs Free Energy (298.15K) are given in Hartree/Particle followed by the Cartesian coordinates are given in Angstrom for each structure.<smiles>c1ccc(-c2ccc(-c3ccccc3)[nH]2)cc1</smiles>

2,5-ph-Py

$3 b$<smiles>COc1c(-c2ccccc2)[nH]c(-c2ccccc2)c1C</smiles>

$3 \mathbf{i}$<smiles>CCSc1c(-c2ccccc2)[nH]c(-c2ccccc2)c1SCC</smiles>

3j<smiles>c1ccc(-c2[nH]c(-c3ccccc3)c3c2SCS3)cc1</smiles>

$3 n$<smiles>c1ccc(-c2[nH]c(-c3ccccc3)c3c2SCCS3)cc1</smiles>

3v

\section{2,5-diphenyl pyrrole}

Total Energy $=-672.3778173$

Thermal correction to Gibbs Free Energy $=0.204351$
C
$-1.136487$
0.680078
$-0.147637$
C
$-0.707513$
1.975171
$-0.413544$
$\mathrm{C}$
0.707493
1.975183
$-0.413555$ 


$\begin{array}{lrrr}\mathrm{C} & 1.136498 & 0.680093 & -0.147677 \\ \mathrm{~N} & 0.000016 & -0.092560 & -0.013322 \\ \mathrm{H} & 0.000056 & -1.026283 & 0.364788 \\ \mathrm{H} & -1.352278 & 2.813620 & -0.633333 \\ \mathrm{H} & 1.352250 & 2.813576 & -0.633577 \\ \mathrm{C} & 2.476621 & 0.119186 & -0.029124 \\ \mathrm{C} & 3.566960 & 0.946131 & 0.295531 \\ \mathrm{C} & 2.719077 & -1.250841 & -0.234704 \\ \mathrm{C} & 4.850267 & 0.421418 & 0.405858 \\ \mathrm{H} & 3.393848 & 2.001346 & 0.479807 \\ \mathrm{C} & 4.002853 & -1.775399 & -0.109492 \\ \mathrm{H} & 1.902470 & -1.904552 & -0.527540 \\ \mathrm{C} & 5.076109 & -0.942997 & 0.209543 \\ \mathrm{H} & & & \\ \mathrm{H} & 5.677418 & 1.077911 & 0.658822 \\ \mathrm{H} & 4.166984 & -2.835973 & -0.275091 \\ \mathrm{H} & 6.077240 & -1.351606 & 0.302988 \\ \mathrm{C} & -2.476611 & 0.119178 & -0.029096 \\ \mathrm{C} & -3.566972 & 0.946137 & 0.295432 \\ \mathrm{H} & -2.719060 & -1.250878 & -0.234543 \\ \mathrm{H} & -4.850290 & 0.421430 & 0.405738 \\ \mathrm{H} & -3.393912 & 2.001368 & 0.479660 \\ \mathrm{H} & & -1.775425 & -0.109363\end{array}$




$$
\begin{array}{llll}
\mathrm{H} & -1.902444 & -1.904625 & -0.527272 \\
\mathrm{C} & -5.076123 & -0.942995 & 0.209523 \\
\mathrm{H} & -5.677443 & 1.077965 & 0.658585 \\
\mathrm{H} & -4.166967 & -2.836015 & -0.274859 \\
\mathrm{H} & -6.077248 & -1.351620 & 0.302933
\end{array}
$$

3b

Total Energy $=-1930.9394232$

Thermal correction to Gibbs Free Energy $=0.350509$
C
$\begin{array}{lll}-1.942763 & -0.820982 & -0.325263\end{array}$
$\mathrm{C}$
$\begin{array}{lll}-1.043227 & 0.009725 & -0.991894\end{array}$
C
$\begin{array}{lll}0.213568 & -0.685956 & -1.077842\end{array}$
C
$0.051054 \quad-1.928218 \quad-0.467417$
$\mathrm{N} \quad-1.248935 \quad-1.971274 \quad-0.009409$
$\begin{array}{llll}\mathrm{H} & -1.693844 & -2.813796 & 0.321677\end{array}$
$\begin{array}{llll}\text { C } & 0.963019 & -3.044937 & -0.214349\end{array}$
$\begin{array}{llll}\text { C } & 0.823971 & -3.810742 & 0.957632\end{array}$
$\begin{array}{llll}\text { C } & 1.983104 & -3.381520 & -1.120148\end{array}$
$\begin{array}{llll}\text { C } & 1.673270 & -4.884427 & 1.212402\end{array}$
$\begin{array}{llll}\mathrm{H} & 0.066460 & -3.541251 & 1.688023\end{array}$
$\begin{array}{llll}\text { C } & 2.836231 & -4.448653 & -0.854975\end{array}$
$\begin{array}{llll}\mathrm{H} & 2.099318 & -2.800827 & -2.026191\end{array}$
$\begin{array}{llll}\text { C } & 2.684708 & -5.207144 & 0.307372\end{array}$ 


\begin{tabular}{|c|c|c|c|}
\hline $\mathrm{H}$ & 1.552778 & -5.460372 & 2.124827 \\
\hline $\mathrm{H}$ & 3.619306 & -4.694502 & -1.565503 \\
\hline $\mathrm{H}$ & 3.351194 & -6.040135 & 0.507498 \\
\hline $\mathrm{C}$ & -3.338018 & -0.662996 & 0.083296 \\
\hline $\mathrm{C}$ & -3.785072 & -1.247709 & 1.282345 \\
\hline $\mathrm{C}$ & -4.259763 & 0.052247 & -0.699056 \\
\hline $\mathrm{C}$ & -5.112255 & -1.125403 & 1.683663 \\
\hline $\mathrm{H}$ & -3.077970 & -1.771224 & 1.919409 \\
\hline $\mathrm{C}$ & -5.583161 & 0.182643 & -0.286933 \\
\hline $\mathrm{H}$ & -3.934738 & 0.497124 & -1.630799 \\
\hline $\mathrm{C}$ & -6.017334 & -0.407218 & 0.901108 \\
\hline $\mathrm{H}$ & -5.436321 & -1.579275 & 2.615200 \\
\hline $\mathrm{H}$ & -6.281522 & 0.739562 & -0.903894 \\
\hline $\mathrm{H}$ & -7.051331 & -0.306582 & 1.215583 \\
\hline $\mathrm{S}$ & -1.369844 & 1.627975 & -1.605372 \\
\hline $\mathrm{S}$ & 1.691085 & -0.028094 & -1.787321 \\
\hline $\mathrm{C}$ & 2.482065 & 0.751365 & -0.374369 \\
\hline $\mathrm{C}$ & 2.579353 & 0.111523 & 0.865302 \\
\hline $\mathrm{C}$ & 3.052897 & 2.013589 & -0.556971 \\
\hline $\mathrm{C}$ & 3.235843 & 0.743967 & 1.918992 \\
\hline $\mathrm{H}$ & 2.142406 & -0.870365 & 1.003684 \\
\hline C & 3.722917 & 2.631200 & 0.497824 \\
\hline
\end{tabular}




$\begin{array}{llll}\mathrm{H} & 2.943558 & 2.519359 & -1.509948 \\ \mathrm{C} & 3.814063 & 2.001708 & 1.738646 \\ \mathrm{H} & 3.304721 & 0.245998 & 2.881834 \\ \mathrm{H} & 4.150668 & 3.618460 & 0.354154 \\ \mathrm{H} & 4.326228 & 2.490010 & 2.561943 \\ \mathrm{C} & -0.470975 & 2.671439 & -0.459324 \\ \mathrm{C} & -0.062614 & 3.928691 & -0.917075 \\ \mathrm{C} & -0.207404 & 2.289580 & 0.858820 \\ \mathrm{C} & 0.594069 & 4.802767 & -0.052087 \\ \mathrm{H} & -0.251415 & 4.215664 & -1.947075 \\ \mathrm{C} & 0.465500 & 3.162530 & 1.709653 \\ \mathrm{H} & -0.512908 & 1.309830 & 1.206893 \\ \mathrm{C} & 0.863658 & 4.422614 & 1.262441 \\ \mathrm{H} & 0.908180 & 5.777431 & -0.413871 \\ \mathrm{H} & 0.687908 & 2.850058 & 2.724778 \\ \mathrm{H} & 1.389776 & 5.097561 & 1.929977\end{array}$

\section{$3 \mathbf{i}$}

Total Energy $=-1547.4081469$

Thermal correction to Gibbs Free Energy $=0.252334$
C
$\begin{array}{lll}-1.138604 & -0.240321 & -0.077480\end{array}$
C
$\begin{array}{lll}-0.715495 & 1.091502 & -0.052734\end{array}$
C
$\begin{array}{lll}0.715397 & 1.091539 & 0.052654\end{array}$ 


$\begin{array}{lrrr}\mathrm{C} & 1.138540 & -0.240286 & 0.077619 \\ \mathrm{~N} & -0.000018 & -1.009933 & 0.000183 \\ \mathrm{H} & -0.000030 & -2.017742 & 0.000399 \\ \mathrm{C} & 2.460505 & -0.868393 & 0.107087 \\ \mathrm{C} & 3.538936 & -0.270709 & 0.783130 \\ \mathrm{C} & 2.673868 & -2.103721 & -0.531921 \\ \mathrm{C} & 4.784077 & -0.891903 & 0.815333 \\ \mathrm{H} & 3.386224 & 0.679594 & 1.280411 \\ \mathrm{C} & 3.918534 & -2.726590 & -0.486376 \\ \mathrm{H} & 1.870123 & -2.564073 & -1.099628 \\ \mathrm{C} & 4.980900 & -2.123097 & 0.186723 \\ \mathrm{H} & 5.603942 & -0.415257 & 1.344138 \\ \mathrm{H} & 4.061570 & -3.677583 & -0.990434 \\ \mathrm{H} & 5.953254 & -2.604523 & 0.217051 \\ \mathrm{H} & -2.460553 & -0.868424 & -0.106943 \\ \mathrm{C} & -3.386177 & 0.679523 & -1.280411 \\ \mathrm{C} & -2.673934 & -2.103774 & 0.532012 \\ \mathrm{C} & -3.538920 & -0.270763 & -0.783109 \\ \mathrm{C} & -3.918596 & -2.726638 & 0.486368 \\ \mathrm{C} & -1.870189 & -2.564141 & 1.099711 \\ \mathrm{H} & -2.784069 & -0.891962 & -0.815401 \\ \mathrm{H} & & -0.186787\end{array}$




$\begin{array}{lrrr}\mathrm{H} & -4.061676 & -3.677632 & 0.990414 \\ \mathrm{H} & -5.603863 & -0.415321 & -1.344318 \\ \mathrm{H} & -5.953262 & -2.604603 & -0.217166 \\ \mathrm{~S} & -1.734475 & 2.538384 & -0.103761 \\ \mathrm{~S} & 1.734384 & 2.538391 & 0.103537 \\ \mathrm{C} & -2.628879 & 2.372688 & 1.487554 \\ \mathrm{H} & -3.259647 & 1.481834 & 1.489849 \\ \mathrm{H} & -1.920195 & 2.335822 & 2.316510 \\ \mathrm{C} & 2.629255 & 2.372055 & -1.487449 \\ \mathrm{H} & 3.259688 & 1.480962 & -1.489313 \\ \mathrm{H} & 1.920838 & 2.335238 & -2.316642 \\ \mathrm{H} & -3.257174 & 3.260784 & 1.586976 \\ \mathrm{H} & 3.257926 & 3.259881 & -1.586913\end{array}$

3j

Total Energy $=-1626.0537628$

Thermal correction to Gibbs Free Energy $=0.304689$
C
$\begin{array}{lll}1.130784 & -0.575391 & -0.154275\end{array}$
C
0.710045
0.756669
$-0.103094$
C
$-0.710087$
0.756660
0.103110
C
$-1.130830 \quad-0.575406$
0.154173
$\mathrm{N}$
$\begin{array}{lll}-0.000024 & -1.345055 & -0.000102\end{array}$
$\mathrm{H}$
0.000006
$-2.352880$
0.000130 


\begin{tabular}{|c|c|c|c|}
\hline $\mathrm{C}$ & -2.448993 & -1.203082 & 0.263125 \\
\hline $\mathrm{C}$ & -2.701385 & -2.436238 & -0.365967 \\
\hline $\mathrm{C}$ & -3.484988 & -0.606867 & 1.003968 \\
\hline $\mathrm{C}$ & -3.941678 & -3.058433 & -0.248513 \\
\hline $\mathrm{H}$ & -1.933493 & -2.895358 & -0.982220 \\
\hline $\mathrm{C}$ & -4.726477 & -1.227361 & 1.108023 \\
\hline $\mathrm{H}$ & -3.302759 & 0.342470 & 1.492560 \\
\hline $\mathrm{C}$ & -4.961634 & -2.456382 & 0.488405 \\
\hline $\mathrm{H}$ & -4.115143 & -4.007707 & -0.746252 \\
\hline $\mathrm{H}$ & -5.513043 & -0.751695 & 1.686060 \\
\hline $\mathrm{H}$ & -5.930889 & -2.937258 & 0.575042 \\
\hline $\mathrm{C}$ & 2.448961 & -1.203053 & -0.263193 \\
\hline $\mathrm{C}$ & 3.484916 & -0.606910 & -1.004144 \\
\hline $\mathrm{C}$ & 2.701381 & -2.436138 & 0.366021 \\
\hline $\mathrm{C}$ & 4.726421 & -1.227387 & -1.108152 \\
\hline $\mathrm{H}$ & 3.302656 & 0.342363 & -1.492845 \\
\hline $\mathrm{C}$ & 3.941684 & -3.058321 & 0.248611 \\
\hline $\mathrm{H}$ & 1.933493 & -2.895222 & 0.982309 \\
\hline $\mathrm{C}$ & 4.961616 & -2.456326 & -0.488389 \\
\hline $\mathrm{H}$ & 5.512957 & -0.751774 & -1.686271 \\
\hline $\mathrm{H}$ & 4.115177 & -4.007539 & 0.746448 \\
\hline $\mathrm{H}$ & 5.930876 & -2.937196 & -0.574991 \\
\hline
\end{tabular}




$\begin{array}{lrrr}\mathrm{S} & 1.726655 & 2.198690 & -0.228068 \\ \mathrm{~S} & -1.726666 & 2.198695 & 0.228161 \\ \mathrm{C} & 2.674180 & 2.075372 & 1.351099 \\ \mathrm{H} & 1.955694 & 2.073601 & 2.174754 \\ \mathrm{H} & 3.212715 & 1.124234 & 1.356133 \\ \mathrm{C} & -2.674232 & 2.075457 & -1.350972 \\ \mathrm{H} & -1.955749 & 2.073399 & -2.174628 \\ \mathrm{H} & -3.213041 & 1.124471 & -1.355922 \\ \mathrm{C} & 3.639857 & 3.250197 & 1.459170 \\ \mathrm{H} & 3.106248 & 4.205190 & 1.447233 \\ \mathrm{H} & 4.204679 & 3.187881 & 2.394661 \\ \mathrm{H} & 4.355681 & 3.250129 & 0.631456 \\ \mathrm{C} & -3.639577 & 3.250549 & -1.459170 \\ \mathrm{H} & -4.355360 & 3.250815 & -0.631420 \\ \mathrm{H} & -3.105683 & 4.205383 & -1.447401 \\ \mathrm{H} & -4.204470 & 3.188249 & -2.394617\end{array}$

\section{3n}

Total Energy $=-1546.200236$

Thermal correction to Gibbs Free Energy $=0.236981$
C
$\begin{array}{lll}-1.140113 & -0.367501 & 0.014080\end{array}$
C
$\begin{array}{lll}-0.714259 & 0.963097 & 0.016432\end{array}$
C
$\begin{array}{lll}0.714235 & 0.963079 & -0.016244\end{array}$ 


\begin{tabular}{|c|c|c|c|}
\hline $\mathrm{C}$ & 1.140070 & -0.367517 & -0.013889 \\
\hline $\mathrm{N}$ & -0.000021 & -1.139514 & 0.000010 \\
\hline $\mathrm{H}$ & -0.000034 & -2.146359 & 0.000146 \\
\hline $\mathrm{C}$ & 2.465130 & -0.983052 & 0.033499 \\
\hline $\mathrm{C}$ & 2.654910 & -2.212141 & 0.692432 \\
\hline $\mathrm{C}$ & 3.572846 & -0.385886 & -0.594321 \\
\hline $\mathrm{C}$ & 3.904040 & -2.826573 & 0.713619 \\
\hline $\mathrm{H}$ & 1.825876 & -2.672381 & 1.222412 \\
\hline $\mathrm{C}$ & 4.822140 & -0.998230 & -0.559763 \\
\hline $\mathrm{H}$ & 3.443391 & 0.551842 & -1.121602 \\
\hline $\mathrm{C}$ & 4.995589 & -2.221935 & 0.089501 \\
\hline $\mathrm{H}$ & 4.027563 & -3.772666 & 1.232026 \\
\hline $\mathrm{H}$ & 5.663548 & -0.521119 & -1.053135 \\
\hline $\mathrm{H}$ & 5.971255 & -2.696970 & 0.112183 \\
\hline $\mathrm{C}$ & -2.465169 & -0.983023 & -0.033425 \\
\hline $\mathrm{C}$ & -3.572961 & -0.385835 & 0.594254 \\
\hline $\mathrm{C}$ & -2.654886 & -2.212150 & -0.692315 \\
\hline $\mathrm{C}$ & -4.822257 & -0.998162 & 0.559556 \\
\hline $\mathrm{H}$ & -3.443565 & 0.551878 & 1.121575 \\
\hline $\mathrm{C}$ & -3.904023 & -2.826559 & -0.713644 \\
\hline $\mathrm{H}$ & -1.825786 & -2.672417 & -1.222167 \\
\hline $\mathrm{C}$ & -4.995645 & -2.221881 & -0.089697 \\
\hline
\end{tabular}




$\begin{array}{llll}\mathrm{H} & -5.663719 & -0.521034 & 1.052819 \\ \mathrm{H} & -4.027494 & -3.772670 & -1.232032 \\ \mathrm{H} & -5.971316 & -2.696902 & -0.112492 \\ \mathrm{~S} & -1.793681 & 2.365129 & -0.089803 \\ \mathrm{~S} & 1.793739 & 2.365056 & 0.089951 \\ \mathrm{C} & -0.650071 & 3.707600 & 0.397839 \\ \mathrm{C} & 0.650274 & 3.707429 & -0.398127 \\ \mathrm{H} & -1.215444 & 4.621917 & 0.193979 \\ \mathrm{H} & 1.215649 & 4.621822 & -0.194591 \\ \mathrm{H} & -0.446714 & 3.657315 & 1.470019 \\ \mathrm{H} & 0.446885 & 3.656831 & -1.470292 \\ \mathbf{3 v} & & & \end{array}$

Total Energy $=-1506.8726458$

Thermal correction to Gibbs Free Energy $=0.209099$
$\begin{array}{llll}\text { C } & -1.143041 & -0.247730 & 0.034283\end{array}$
$\begin{array}{llll}\text { C } & -0.712667 & 1.073130 & 0.059440\end{array}$
$\begin{array}{llll}\text { C } & 0.707574 & 1.080953 & 0.044389\end{array}$
$\begin{array}{llll}\text { C } & 1.152170 & -0.235783 & 0.026742\end{array}$
$\begin{array}{llll}\mathrm{N} & 0.008637 & -1.017216 & 0.027153\end{array}$
$\mathrm{H} \quad 0.013723 \quad-2.022725 \quad-0.010423$
$\begin{array}{llll}\text { C } & 2.490333 & -0.813455 & 0.035437\end{array}$
$\begin{array}{llll}\text { C } & 2.712158 & -2.130350 & 0.477777\end{array}$ 


$\begin{array}{llll}\mathrm{C} & 3.595741 & -0.063990 & -0.406636 \\ \mathrm{C} & 3.990442 & -2.681480 & 0.465788 \\ \mathrm{H} & 1.886984 & -2.719251 & 0.867906 \\ \mathrm{C} & 4.872301 & -0.616481 & -0.408578 \\ \mathrm{H} & 3.444368 & 0.947608 & -0.767242 \\ \mathrm{C} & 5.078306 & -1.928291 & 0.023389 \\ \mathrm{H} & 4.138167 & -3.698589 & 0.815948 \\ \mathrm{H} & 5.710728 & -0.020973 & -0.756834 \\ \mathrm{H} & 6.075538 & -2.356490 & 0.020095 \\ \mathrm{C} & -2.477017 & -0.828039 & -0.010087 \\ \mathrm{C} & -3.584777 & -0.108055 & 0.472432 \\ \mathrm{C} & -2.697072 & -2.114140 & -0.537362 \\ \mathrm{C} & -4.863981 & -0.653319 & 0.421514 \\ \mathrm{H} & -3.437191 & 0.871825 & 0.913580 \\ \mathrm{C} & -3.976165 & -2.660795 & -0.573863 \\ \mathrm{H} & -1.868460 & -2.678123 & -0.956144 \\ \mathrm{H} & -5.067649 & -1.933087 & -0.097037 \\ \mathrm{H} & -5.704252 & -0.079800 & 0.800578 \\ \mathrm{H} & -4.122811 & -3.653340 & -0.988932 \\ \mathrm{H} & -6.065594 & -2.358266 & -0.130801 \\ \mathrm{H} & -1.526806 & 2.637322 & 0.117401 \\ \mathrm{H} & & 2.658749 & 0.138918\end{array}$




$\begin{array}{llll}\mathrm{C} & -0.015078 & 3.532948 & -0.449255 \\ \mathrm{H} & -0.009408 & 3.580072 & -1.539627 \\ \mathrm{H} & -0.024614 & 4.539215 & -0.029504\end{array}$

X-ray crystal data of 3a (CCDC 2092060, thermal ellipsoids are set at a $50 \%$ probability level)

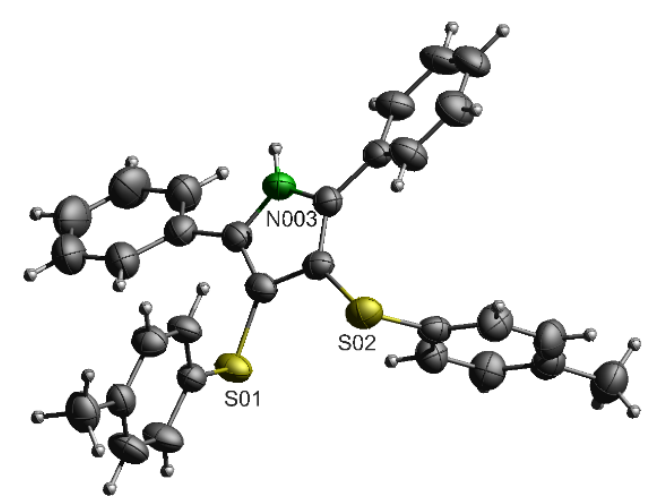

\begin{tabular}{|l|l|}
\hline Crystal data \\
\hline Chemical formula & $\mathrm{C}_{30} \mathrm{H}_{25} \mathrm{NS}_{2}$ \\
\hline$M_{\mathrm{r}}$ & $\underline{463.63}$ \\
\hline $\begin{array}{l}\text { Crystal system, } \\
\text { space group }\end{array}$ & Triclinic, $\underline{P}$ \\
\hline
\end{tabular}




\begin{tabular}{|c|c|}
\hline Temperature (K) & 295 \\
\hline$a, b, c(\AA)$ & $7.8739(5), \underline{12.1667(8)}, \underline{14.0951(10)}$ \\
\hline$\alpha, \beta, \gamma\left({ }^{\circ}\right)$ & $105.585(2), \underline{97.216(2)}, \underline{105.644(2)}$ \\
\hline$V\left(\AA^{3}\right)$ & $1223.59(14)$ \\
\hline$Z$ & $\underline{2}$ \\
\hline Radiation type & Mo Ka \\
\hline$\mu\left(\mathrm{mm}^{-1}\right)$ & 0.24 \\
\hline \multicolumn{2}{|l|}{ Data collection } \\
\hline Diffractometer & Bruker APEX-II CCD \\
\hline $\begin{array}{l}\text { Absorption } \\
\text { correction }\end{array}$ & $\begin{array}{l}\text { Multi-scan } \\
\text { SADABS2016/2 (Bruker,2016/2) was used for absorption correction. } \\
\text { wR2(int) was } 0.0629 \text { before and } 0.0486 \text { after correction. The Ratio of } \\
\text { minimum to maximum transmission is } 0.8748 \text {. The } \lambda / 2 \text { correction factor } \\
\text { is Not present. }\end{array}$ \\
\hline$T_{\min }, T_{\max }$ & $\underline{0.652}, \underline{0.746}$ \\
\hline $\begin{array}{l}\text { No. of measured, } \\
\text { independent and } \\
\text { observed }[\underline{I>2 \sigma(I)]} \\
\text { reflections }\end{array}$ & $\underline{9510}, \underline{4201}, \underline{3054}$ \\
\hline$R_{\text {int }}$ & 0.029 \\
\hline$(\sin \theta / \lambda)_{\max }\left(\AA^{-1}\right)$ & 0.595 \\
\hline \multicolumn{2}{|l|}{ Refinement } \\
\hline $\begin{array}{l}R\left[F^{2}>2 \sigma\left(F^{2}\right)\right] \\
w R\left(F^{2}\right), S\end{array}$ & $\underline{0.049}, \underline{0.123}, \underline{1.04}$ \\
\hline No. of reflections & $\underline{4201}$ \\
\hline No. of parameters & $\underline{300}$ \\
\hline H-atom treatment & H-atom parameters constrained \\
\hline$\Delta \rho_{\max }, \Delta \rho_{\min }\left(\mathrm{e} \AA^{-3}\right)$ & $0.23,-0.23$ \\
\hline
\end{tabular}

Computer programs: SAINT v8.37A (Bruker, 2015), XT (Sheldrick, 2015), XL (Sheldrick, 2008), Olex2 (Dolomanov et al., 2009). 
$3 a$

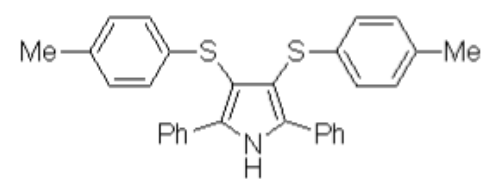

${ }^{1} \mathrm{HNMR} 400 \mathrm{MHz} \mathrm{CDCl}_{3}$

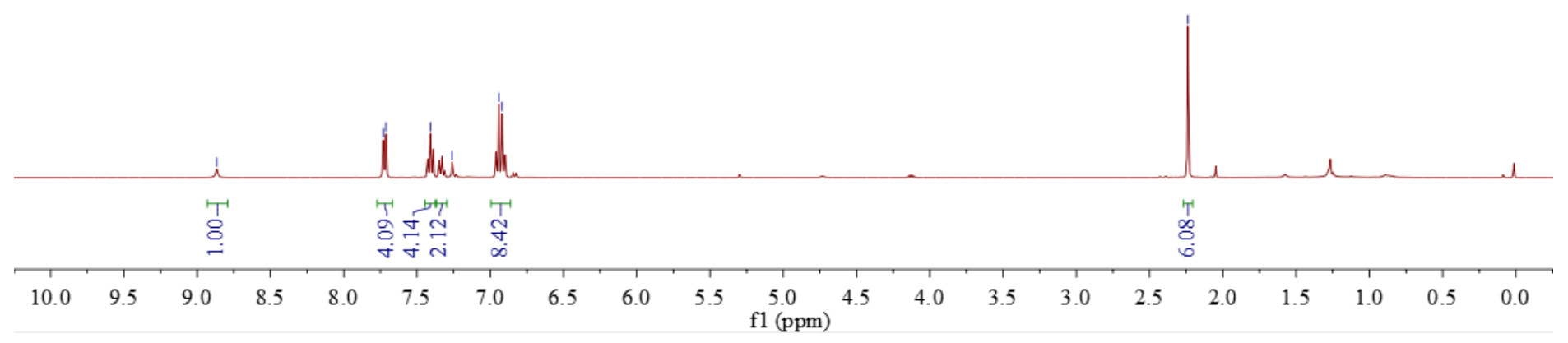




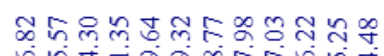
minm

盗

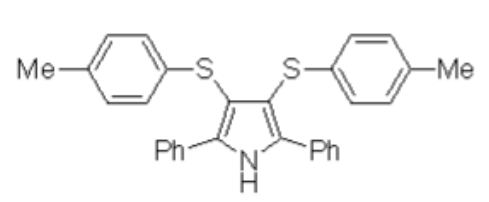

${ }^{13} \mathrm{C}\left\{{ }^{1} \mathrm{H}\right\} \mathrm{NMR} \quad 100 \mathrm{MHz} \quad \mathrm{CDCl}_{3}$
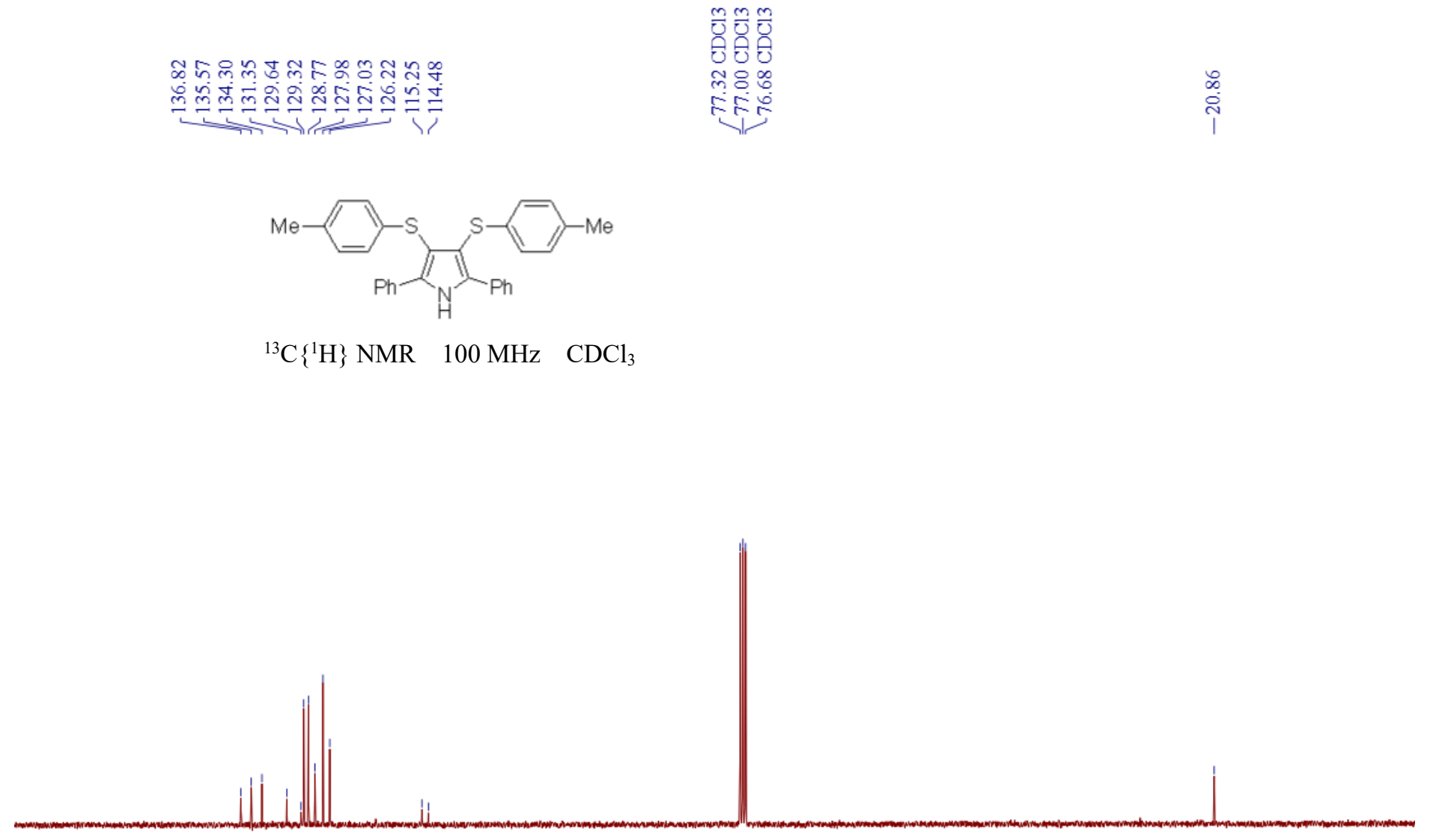


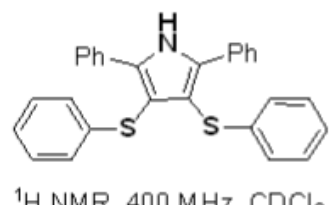

${ }^{1} \mathrm{H} \mathrm{NMR} 400 \mathrm{MHz}^{\circ \mathrm{CDCl}_{3}}$

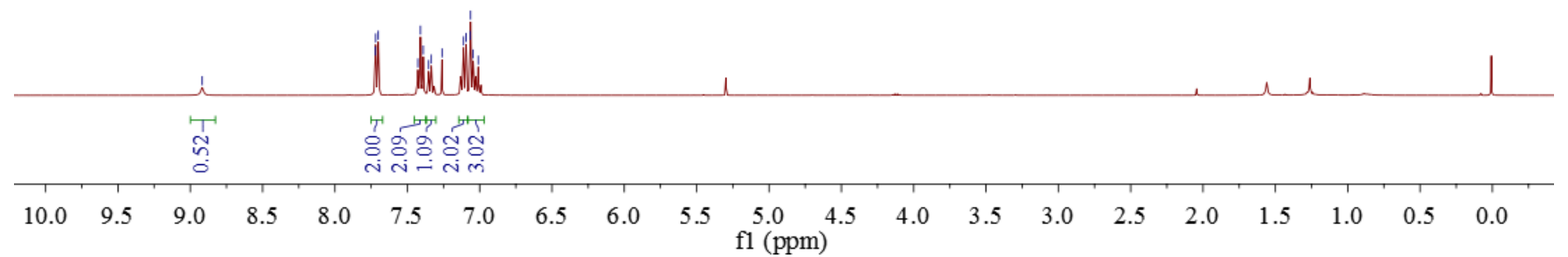



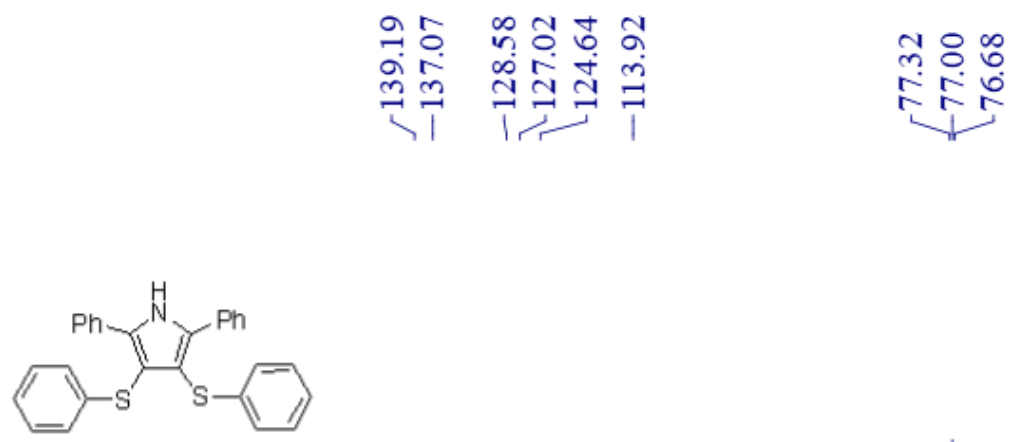

${ }^{13} \mathrm{C}\left\{{ }^{1} \mathrm{H}\right\} \mathrm{NMR} \quad 100 \mathrm{MHz} \quad \mathrm{CDCl}_{3}$
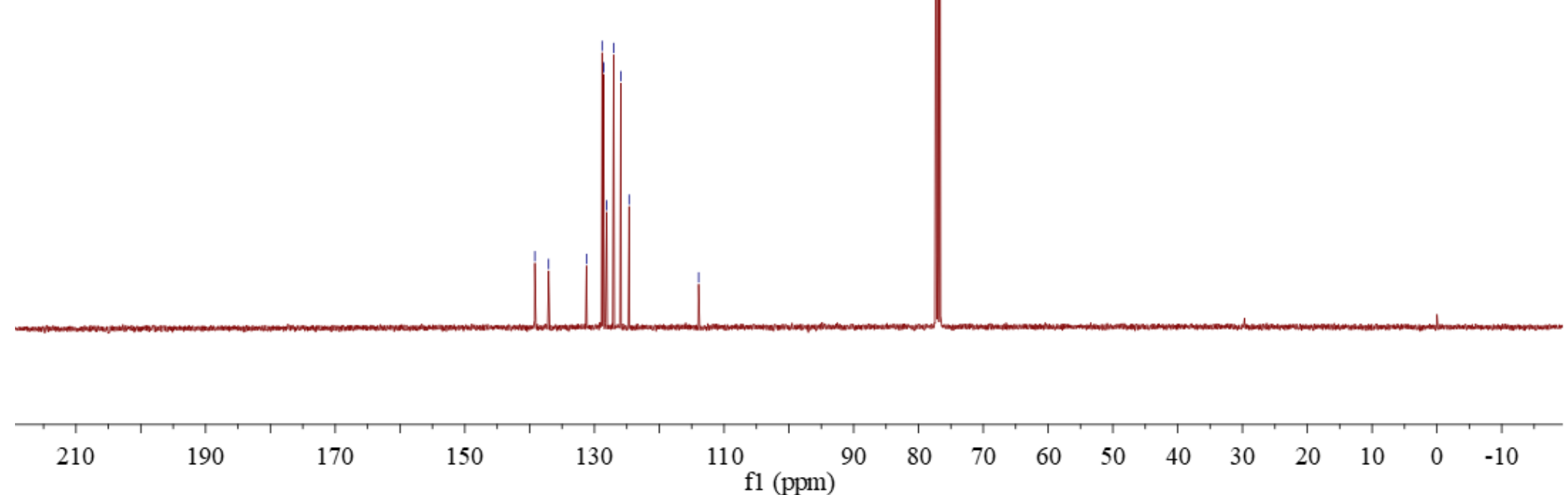
3c

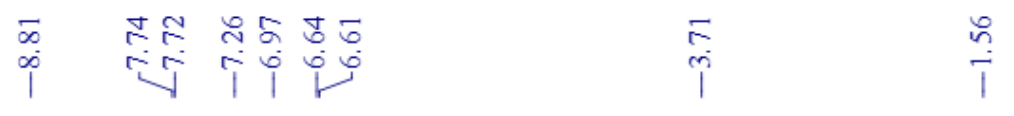

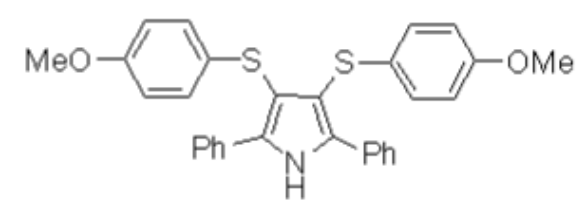

${ }^{1} \mathrm{HNMR} \quad 400 \mathrm{MHz} \quad \mathrm{CDCl}_{3}$

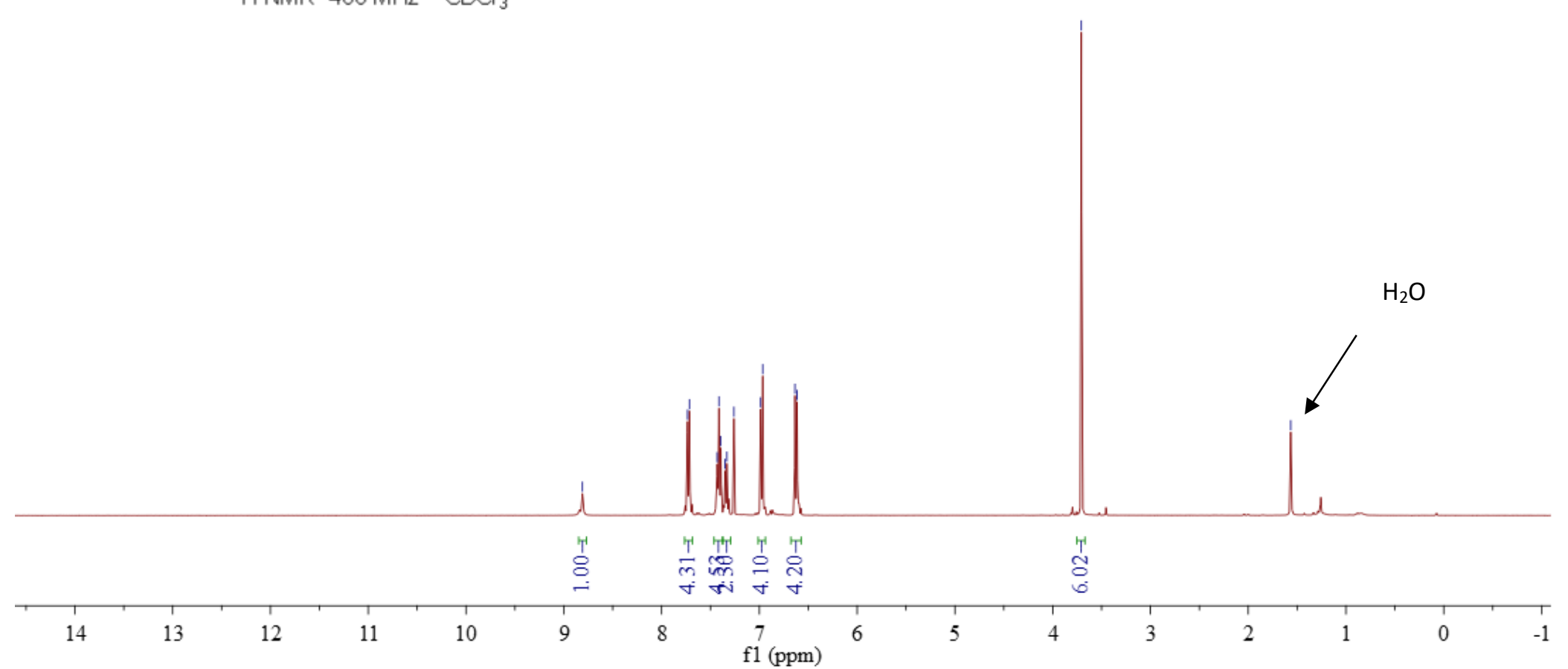



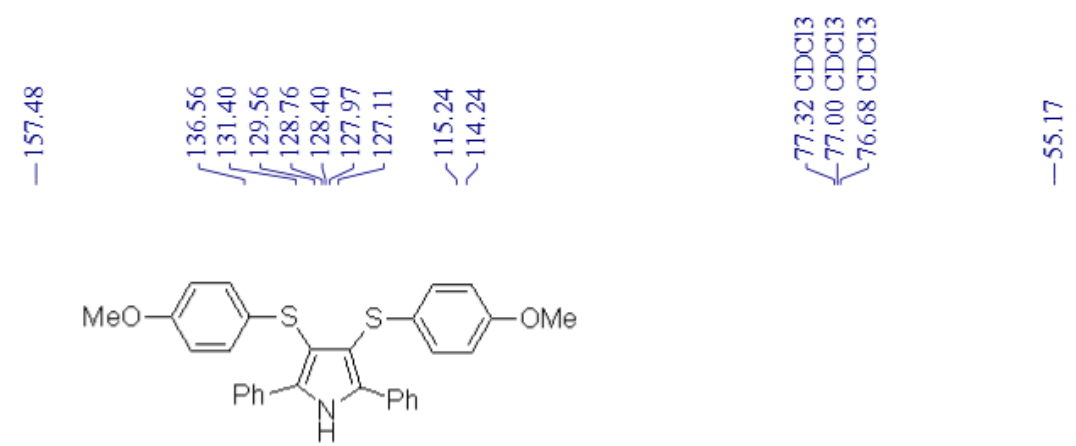

${ }^{13} \mathrm{C}\left\{{ }^{1} \mathrm{H}\right\} \mathrm{NMR} \quad 100 \mathrm{MHz} \quad \mathrm{CDCl}_{3}$

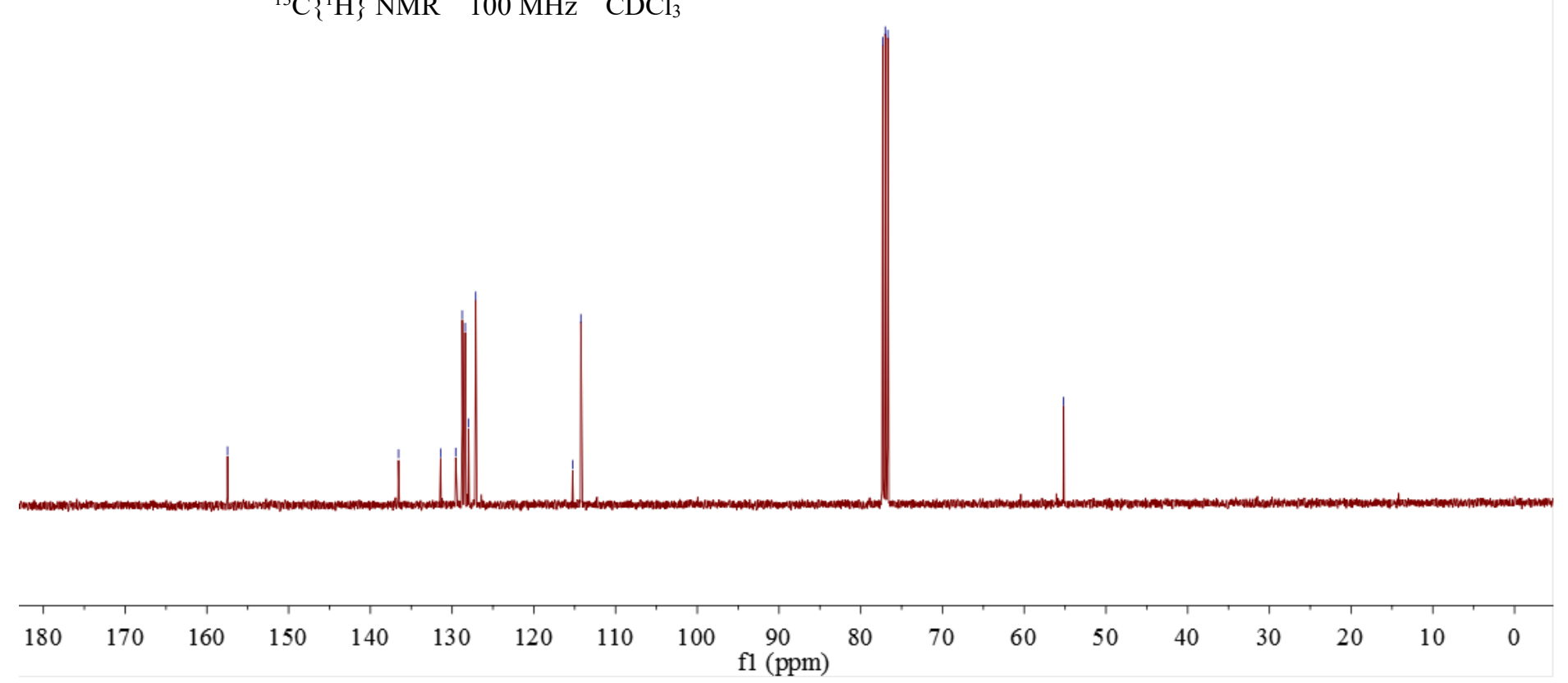




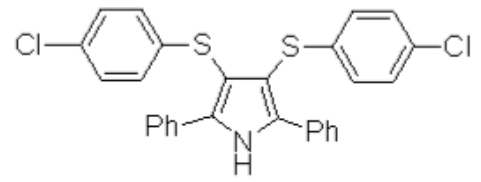

${ }^{1} \mathrm{HNMR} \quad 400 \mathrm{MHz} \quad \mathrm{CDCl}_{3}$

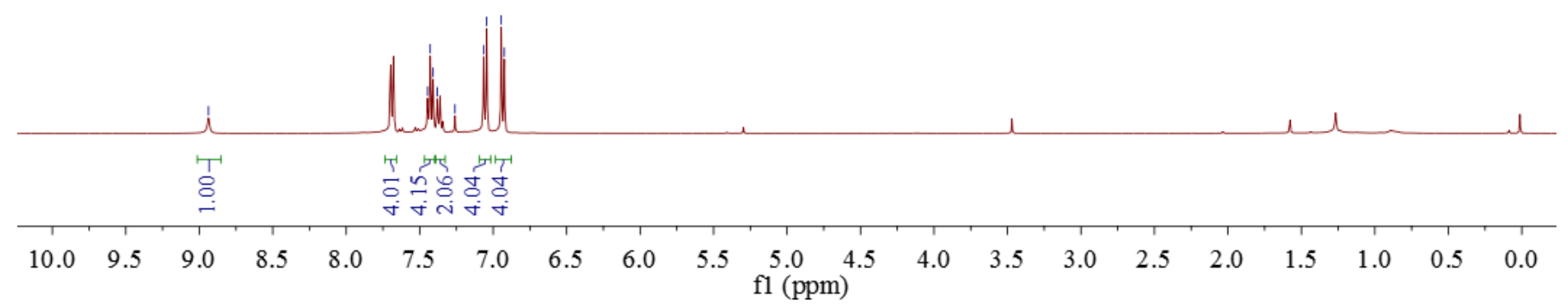



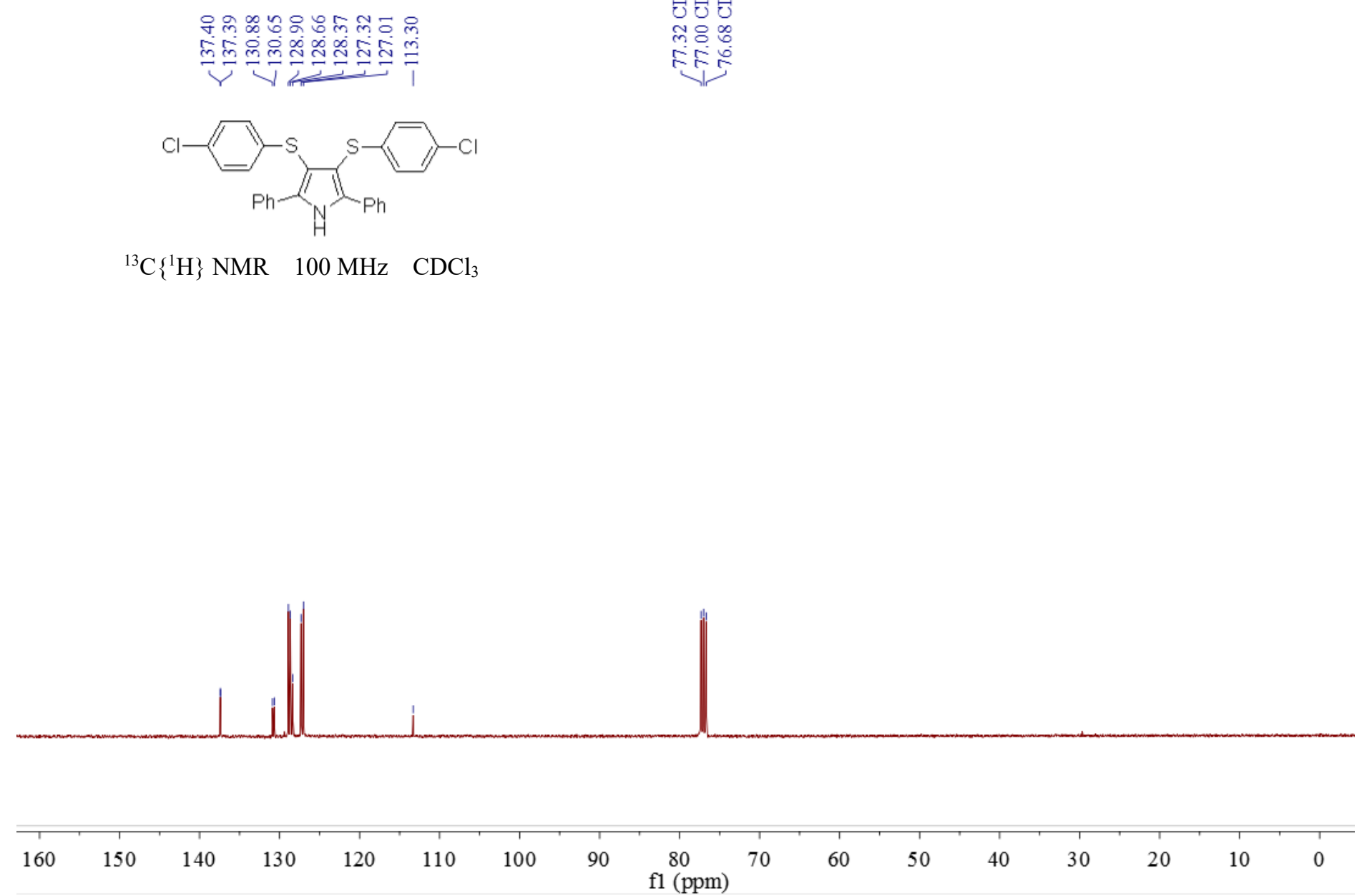
$3 e$

I. $\quad \infty$ ○

i

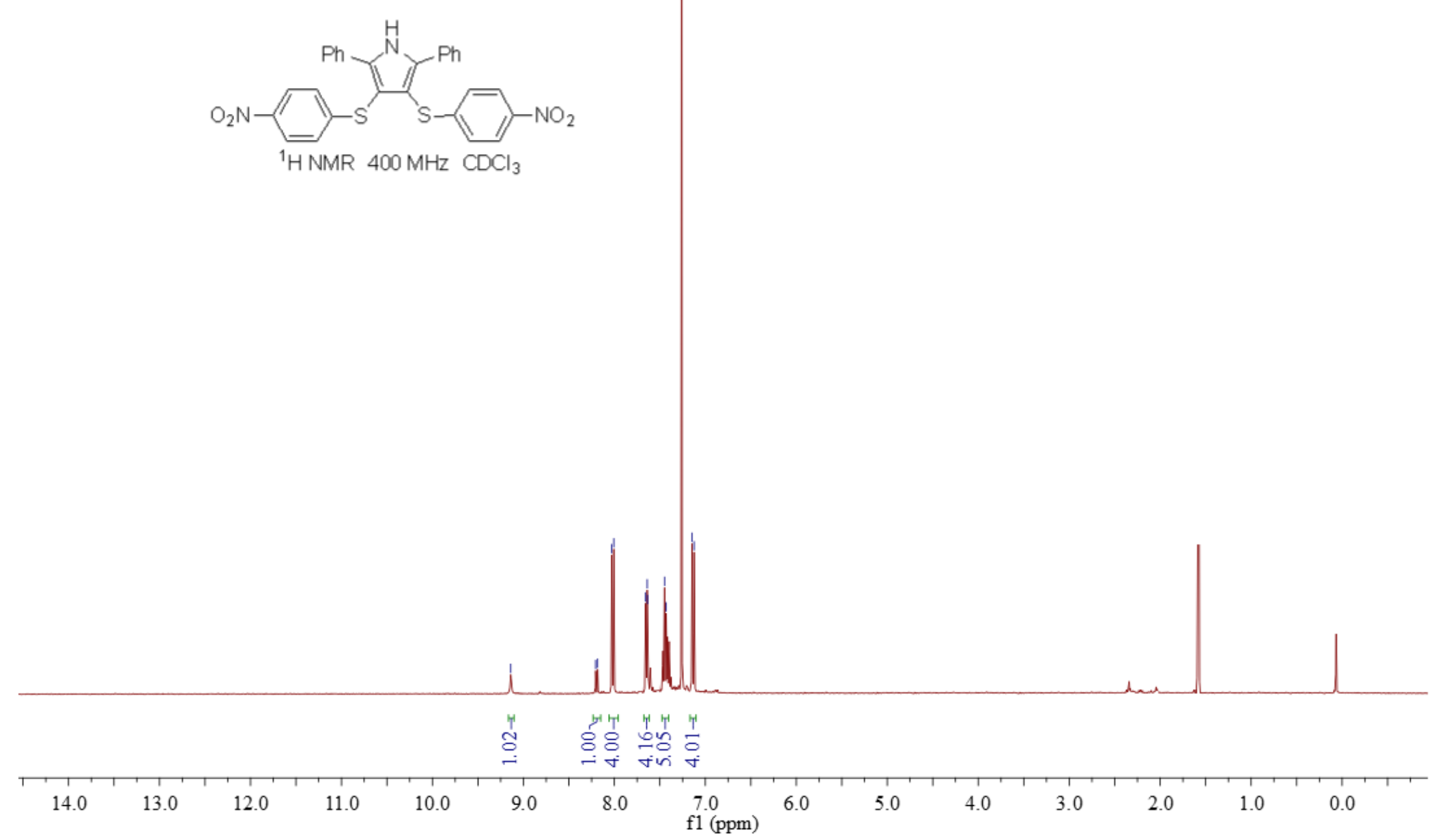




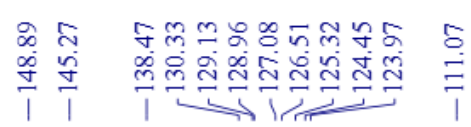
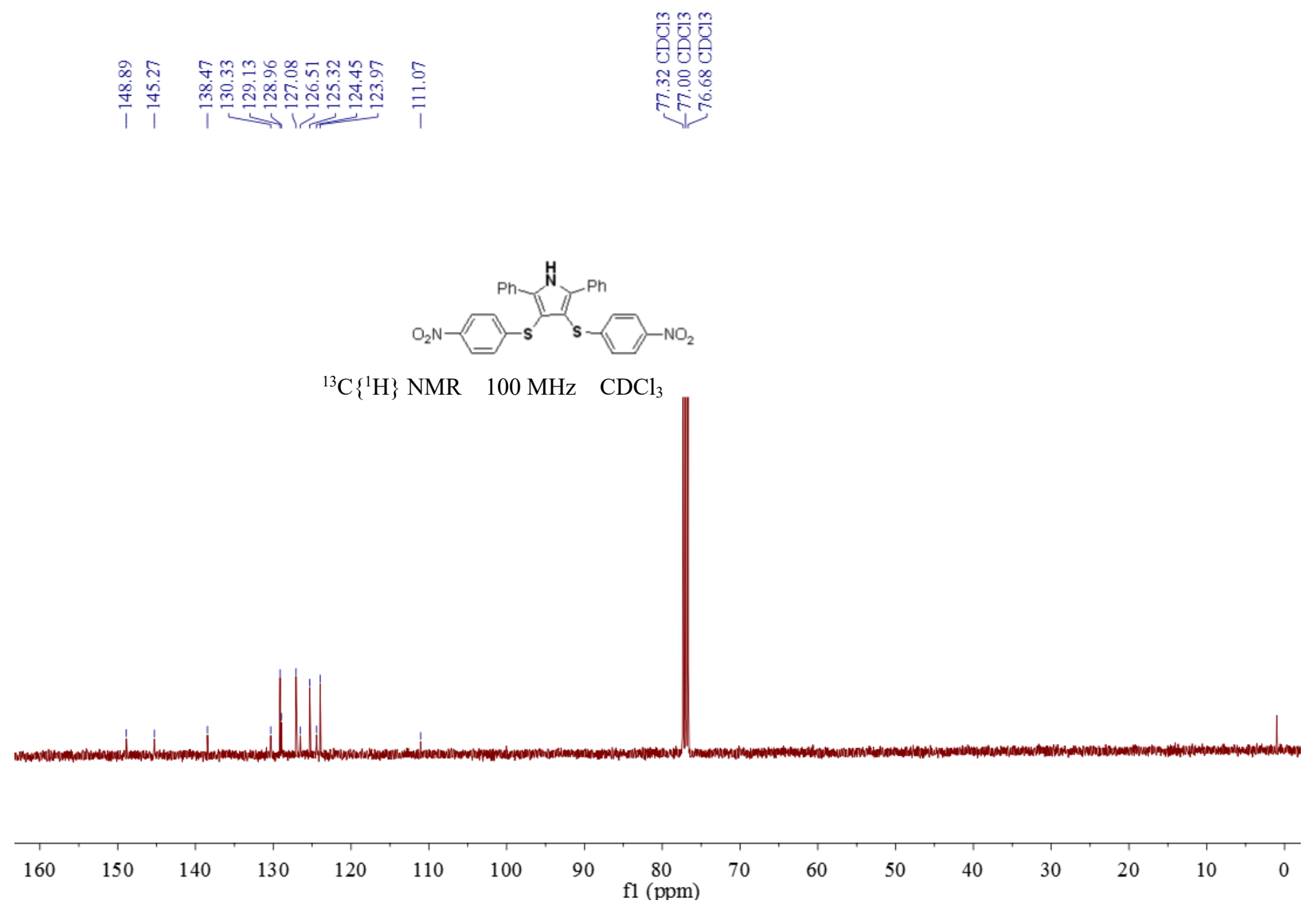


\section{$3 f$}
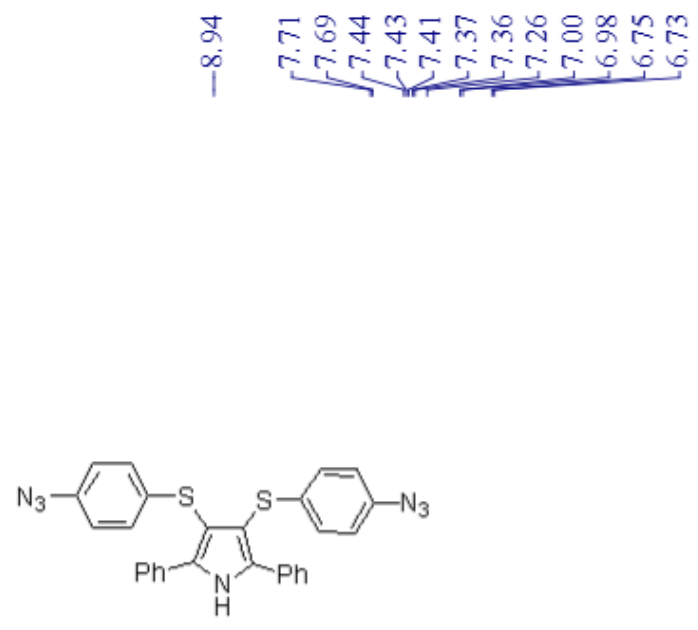

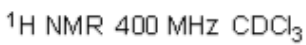

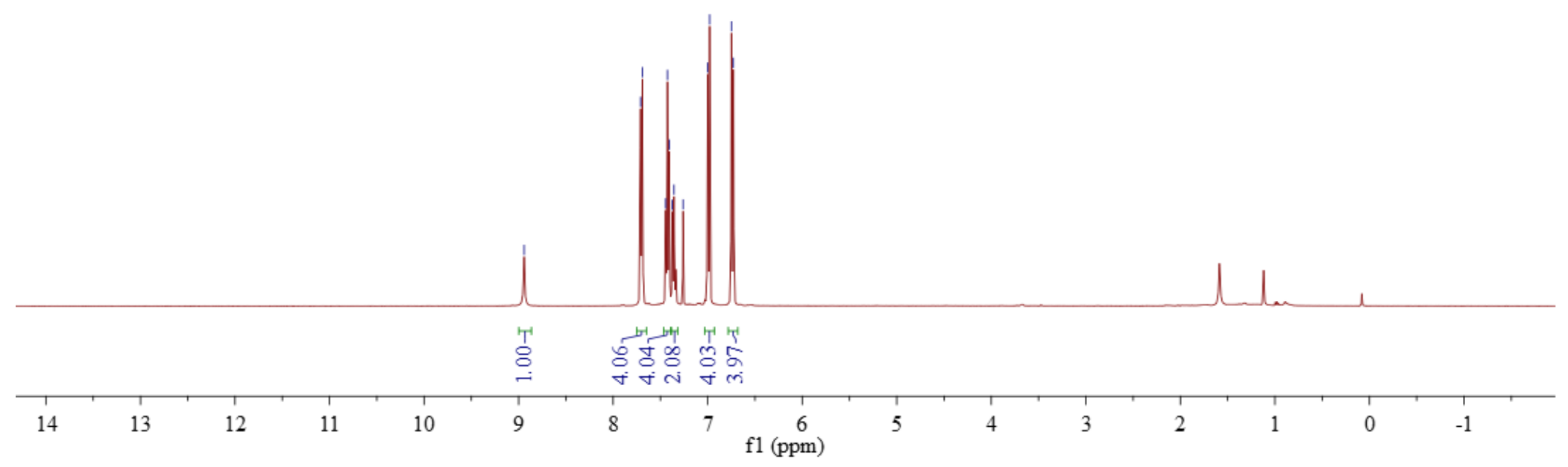




\section{Copies of NMR spectra}

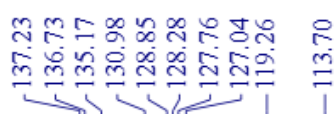

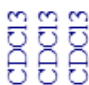

त $\infty$

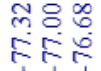

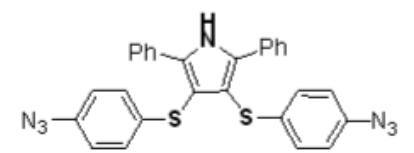

${ }^{13} \mathrm{C}\left\{{ }^{1} \mathrm{H}\right\} \mathrm{NMR} \quad 100 \mathrm{MHz} \quad \mathrm{CDCl}_{3}$

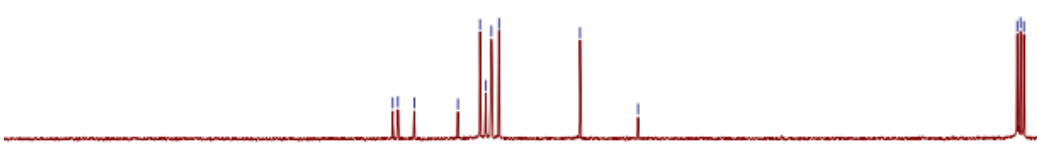

$\begin{array}{rrrrrrrrrr}170 & 160 & 150 & 140 & 130 & 120 & 110 & 100 & 90 & 80 \\ \text { f1 (ppm) }\end{array}$

60 
$3 g$

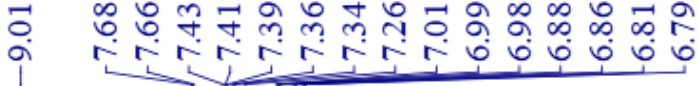

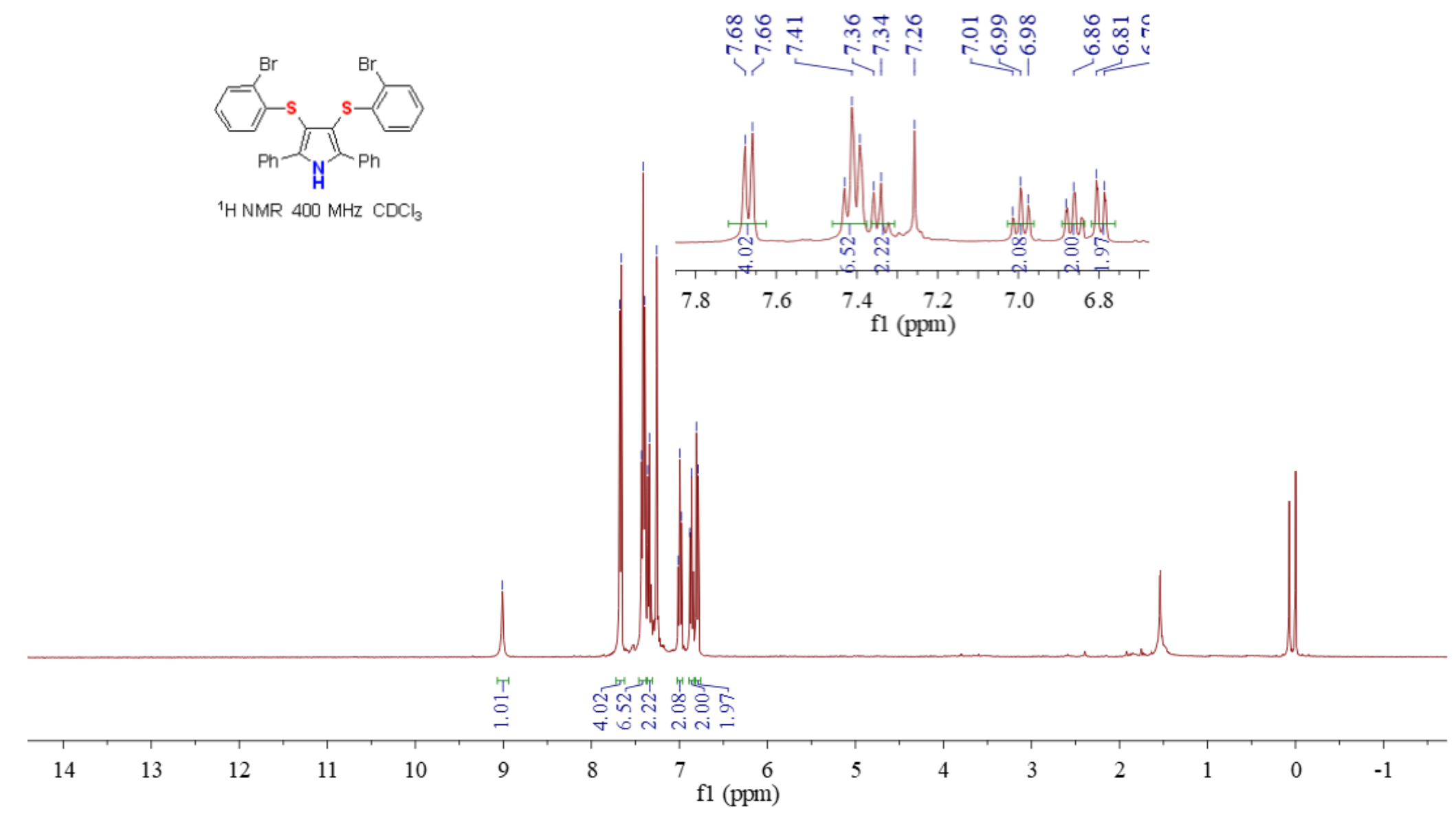




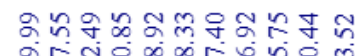

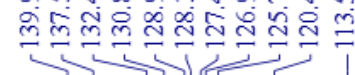

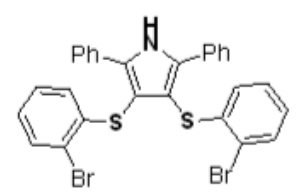

${ }^{13} \mathrm{C}\left\{{ }^{1} \mathrm{H}\right\} \mathrm{NMR} \quad 100 \mathrm{MHz} \quad \mathrm{CDCl}_{3}$

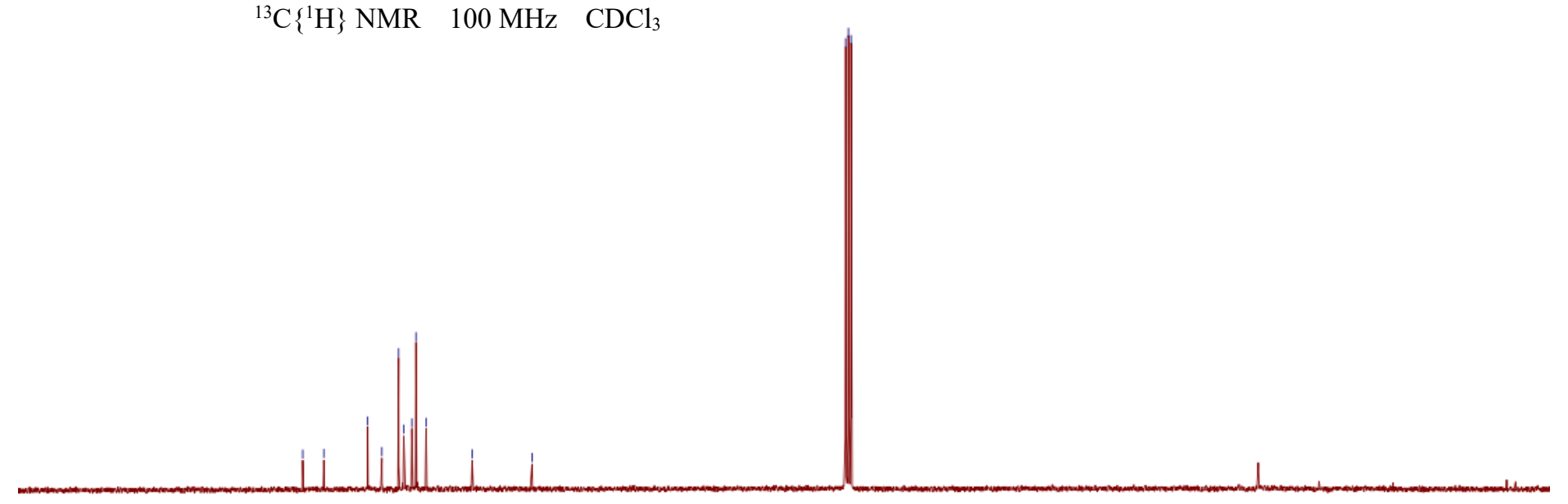

170

$160 \quad 150$

$140 \quad 130$

120

$110 \quad 100$

$90 \quad 80$

$60 \quad 50$

40

30

20 
$3 h$

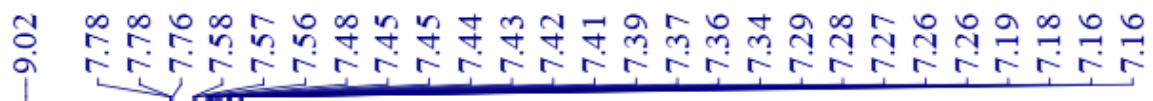

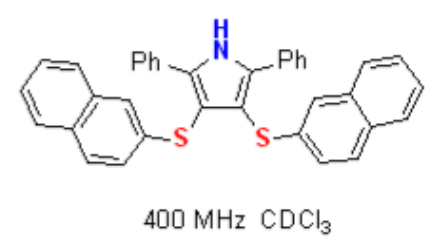

促
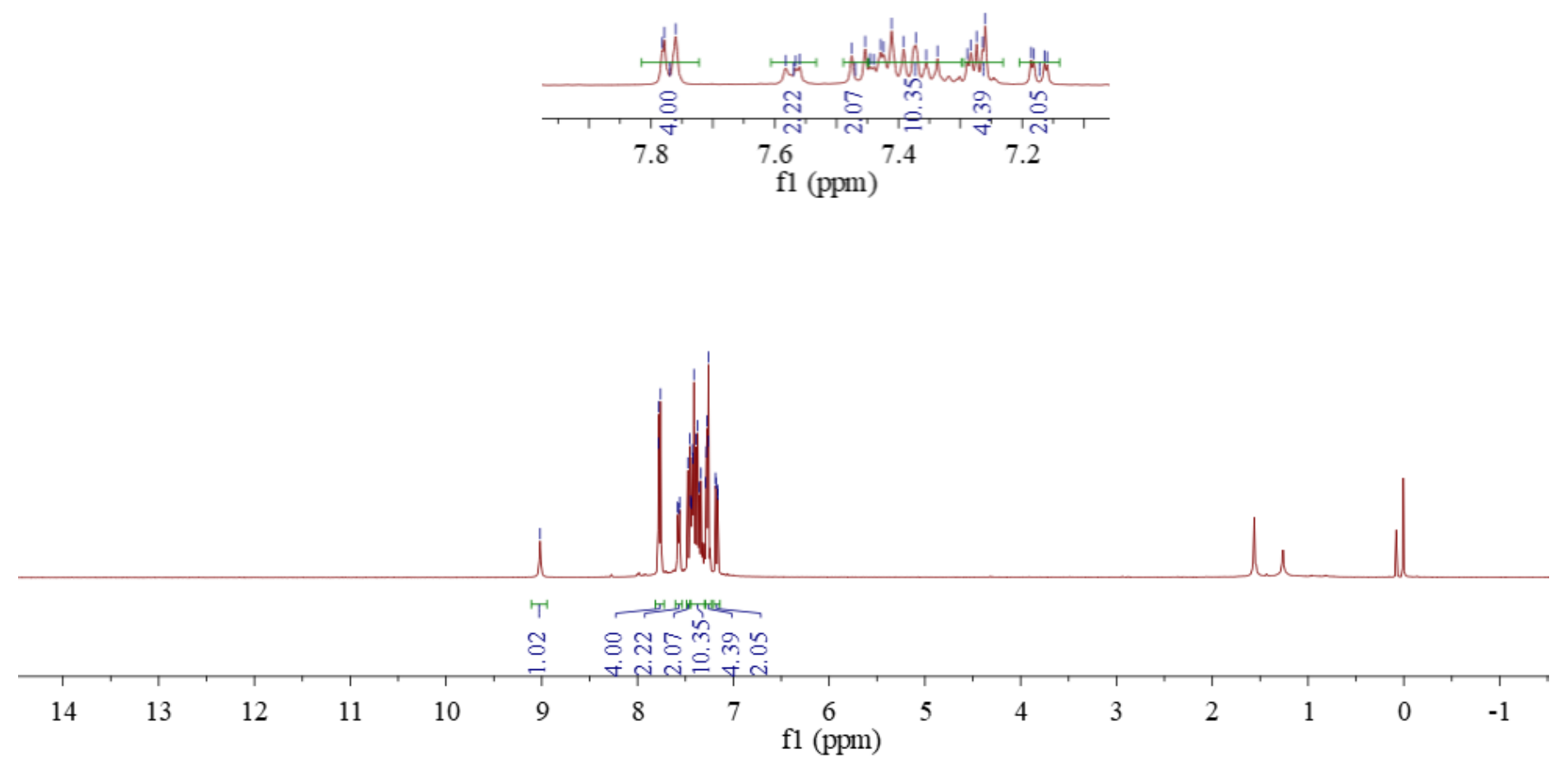
กำกำ

m心

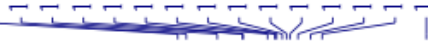

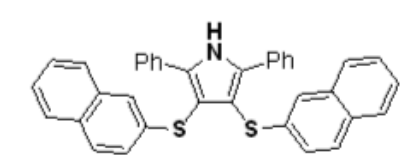

${ }^{13} \mathrm{C}\left\{{ }^{1} \mathrm{H}\right\} \mathrm{NMR} \quad 100 \mathrm{MHz} \quad \mathrm{CDCl}_{3}$

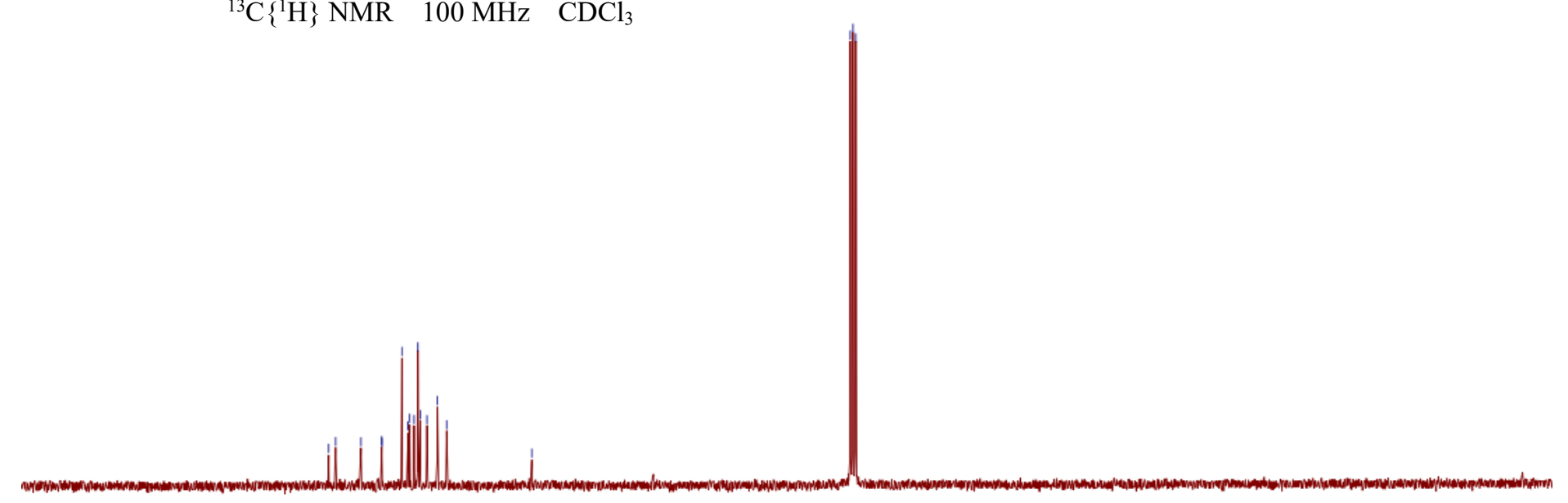

170

$160 \quad 150$

140

130

$110 \quad 100$

$90 \quad 80$

$60 \quad 50$

40

30

20

$10+0$

\author{
(ppm)
}




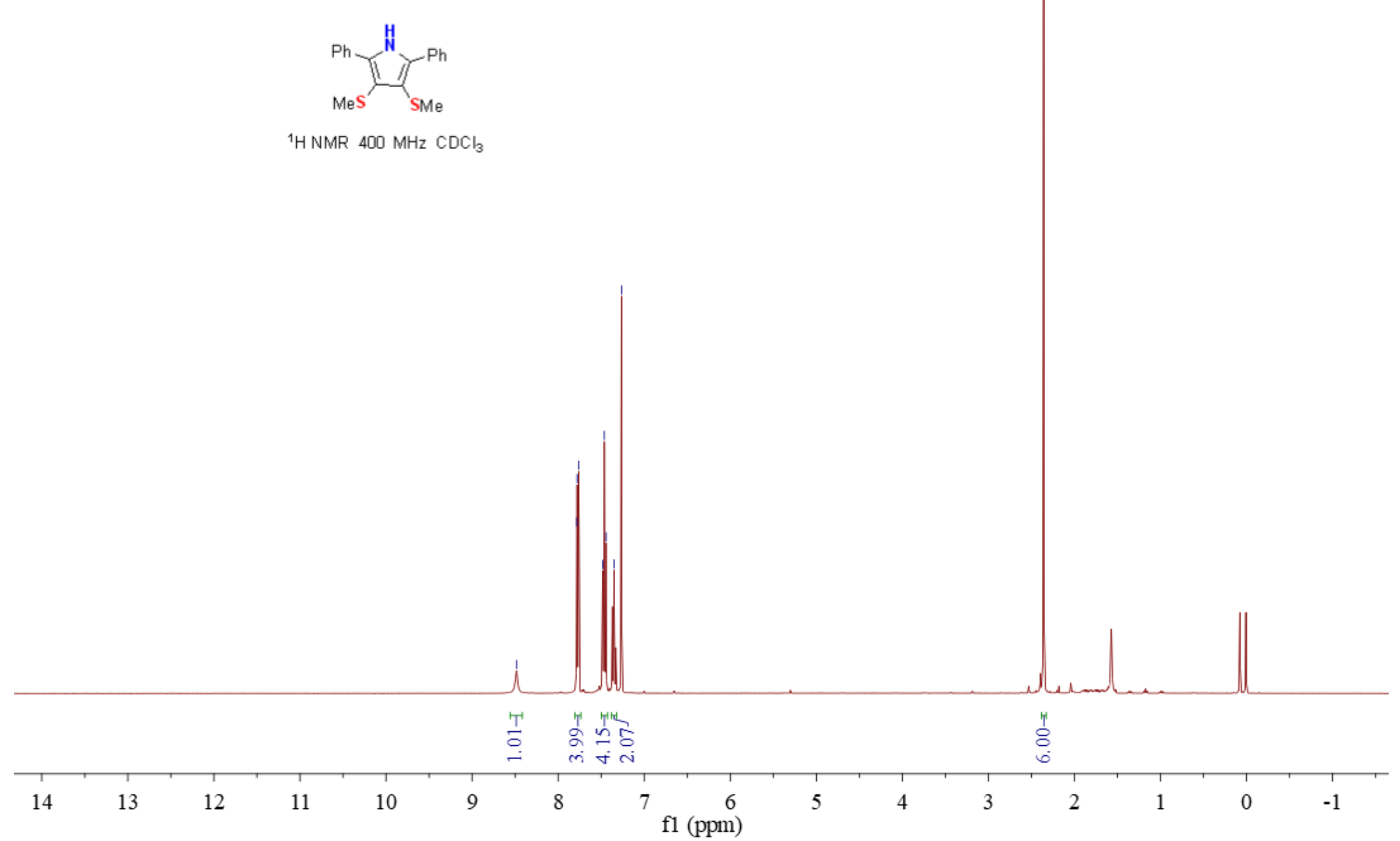




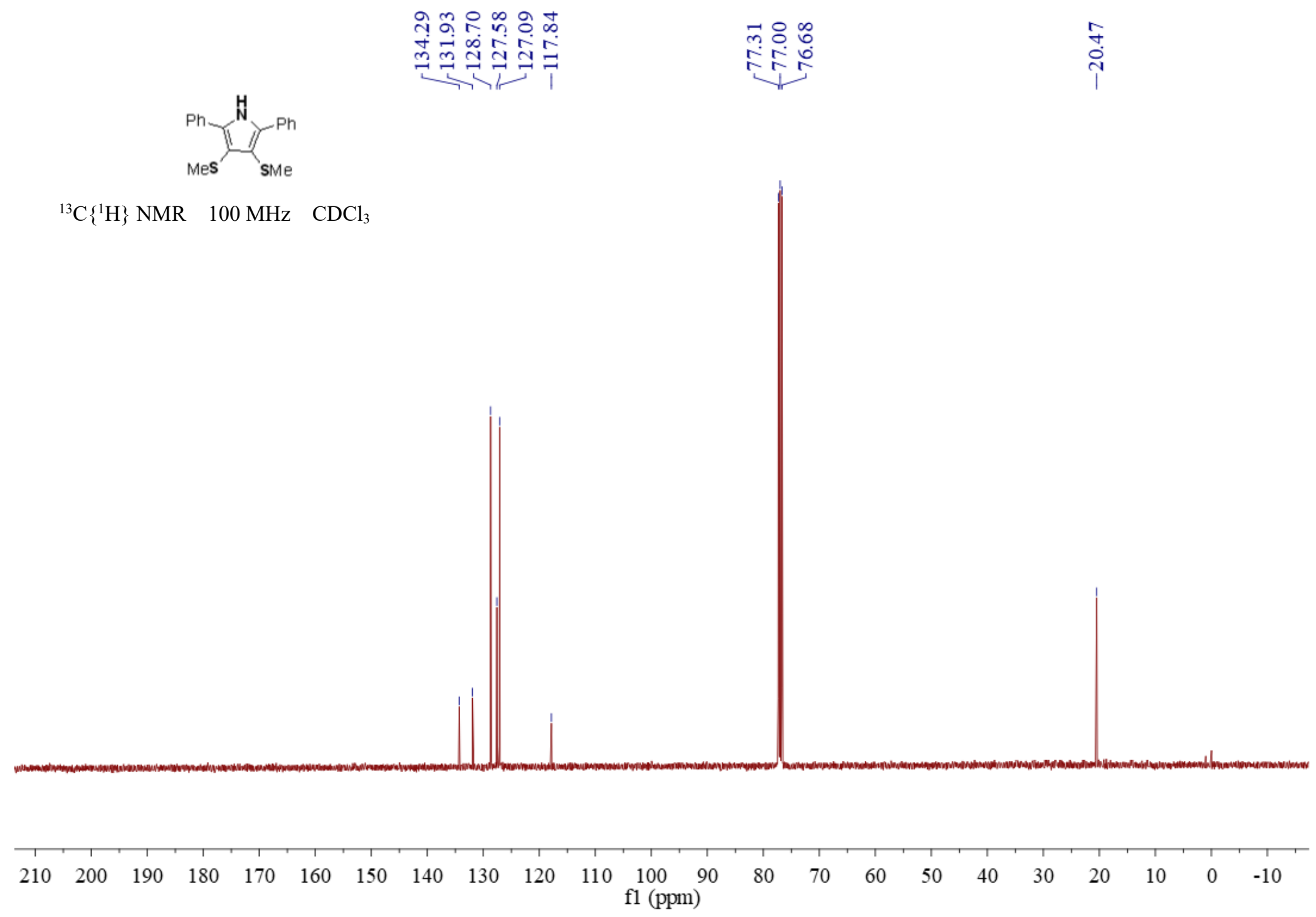


3j

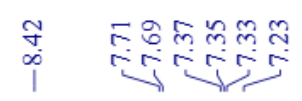

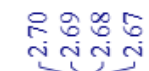

过

$$
{ }^{1} \mathrm{HNMR} \quad 400 \mathrm{MHz} \mathrm{CDCl}_{3}
$$

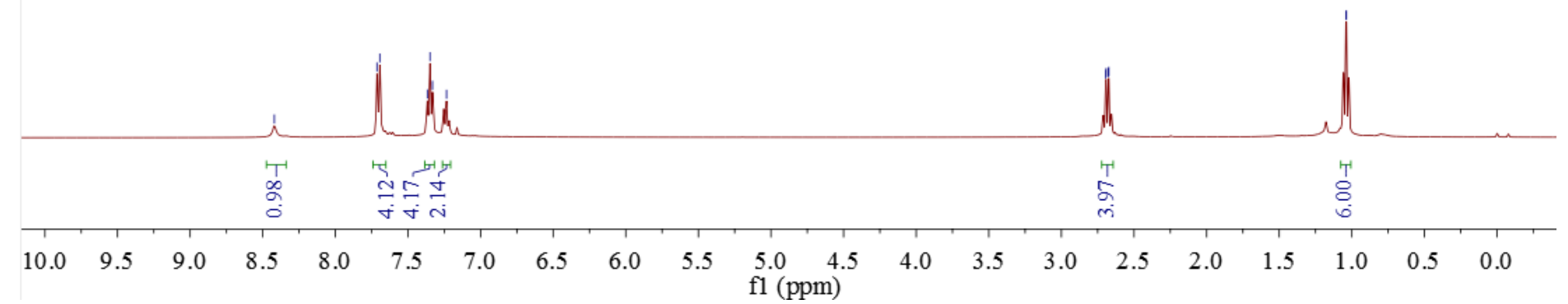




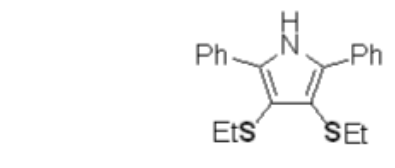

${ }^{13} \mathrm{C}\left\{{ }^{1} \mathrm{H}\right\}$ NMR $\quad 100 \mathrm{MHz} \quad \mathrm{CDCl}_{3}$
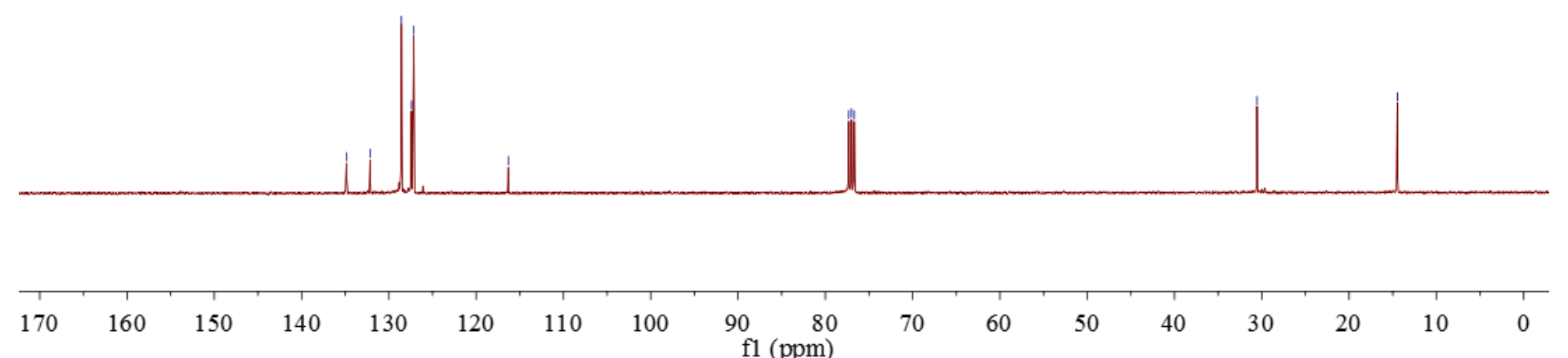
3k
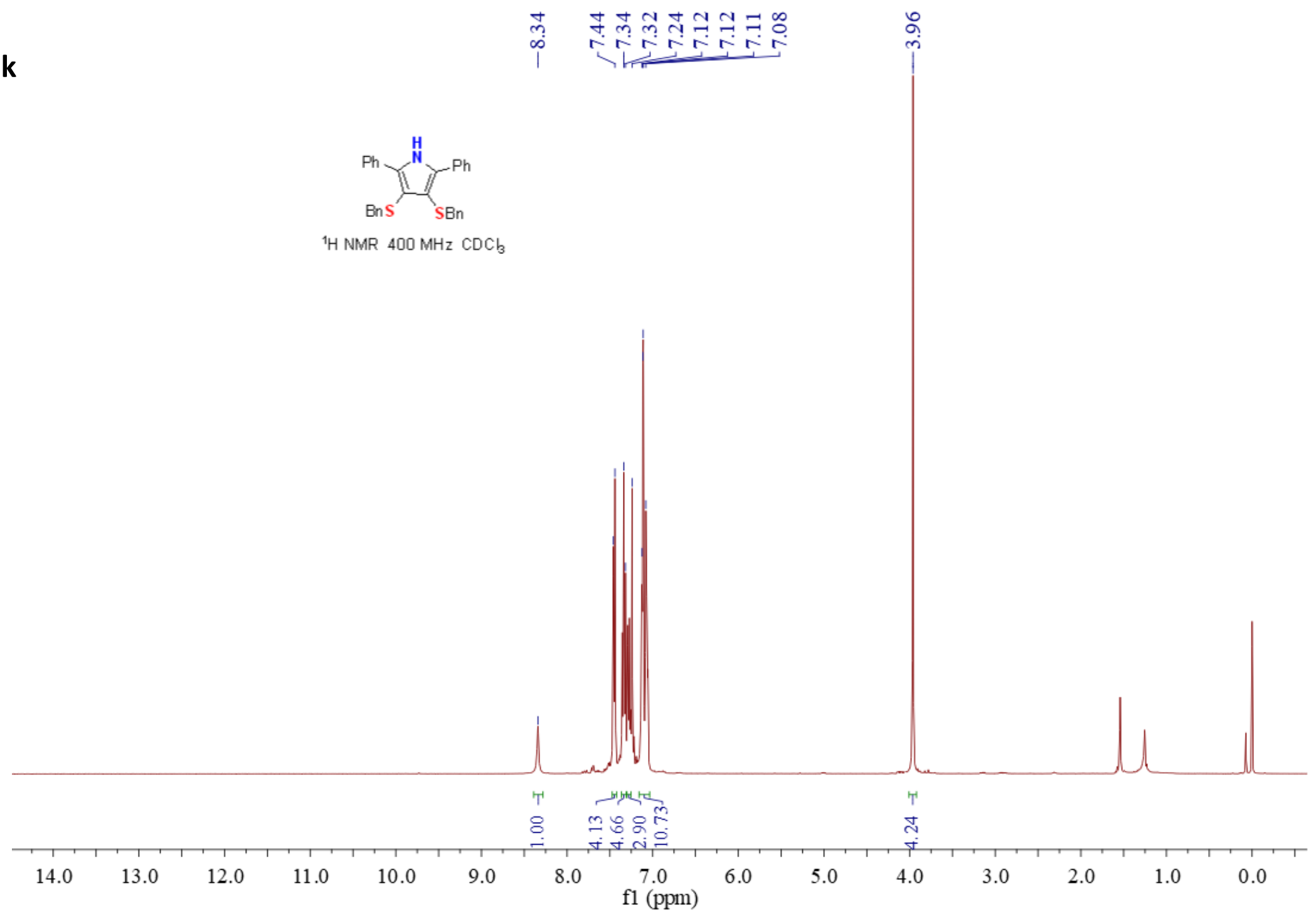


\section{Copies of NMR spectra}

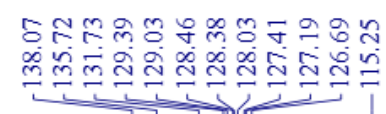
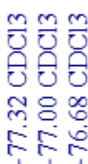

然

$\stackrel{\curvearrowright}{\stackrel{\%}{广}}$

$$
\text { SBn }
$$

${ }^{13} \mathrm{C}\left\{{ }^{1} \mathrm{H}\right\} \mathrm{NMR} \quad 100 \mathrm{MHz} \quad \mathrm{CDCl}_{3}$

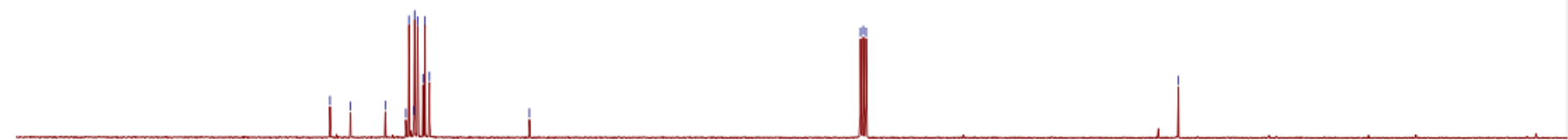

$\begin{array}{lllll}170 & 160 & 150 & 140 & 130\end{array}$

$\begin{array}{lll}120 & 110 & 100\end{array}$

90
$\mathrm{fl}(\mathrm{ppm})$ 


\section{Copies of NMR spectra}
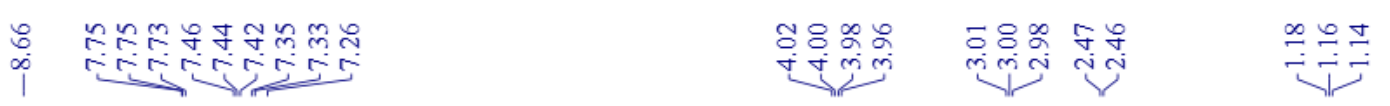

31

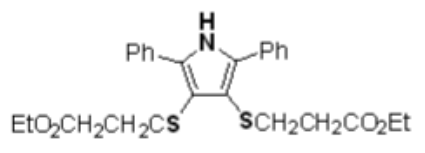

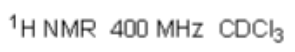

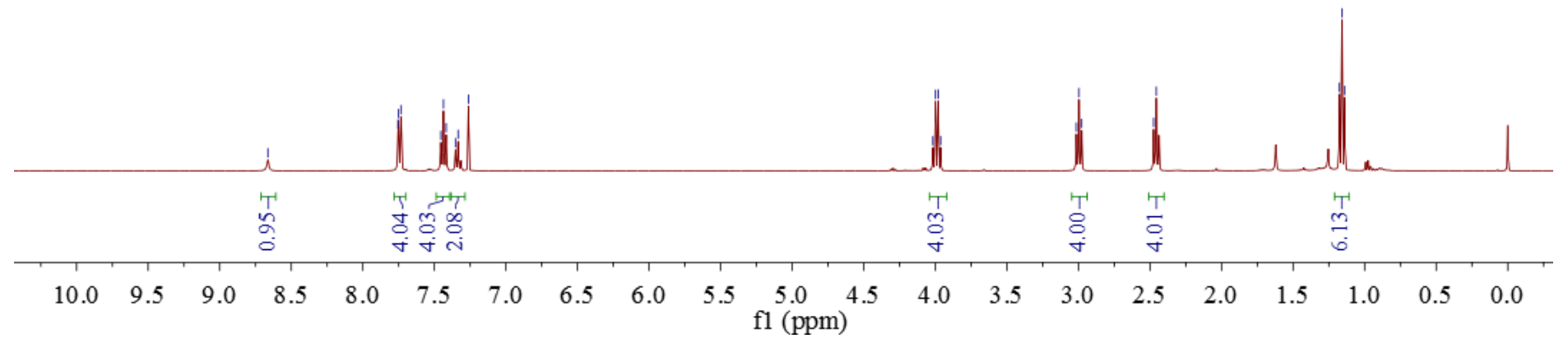




\section{Copies of NMR spectra}
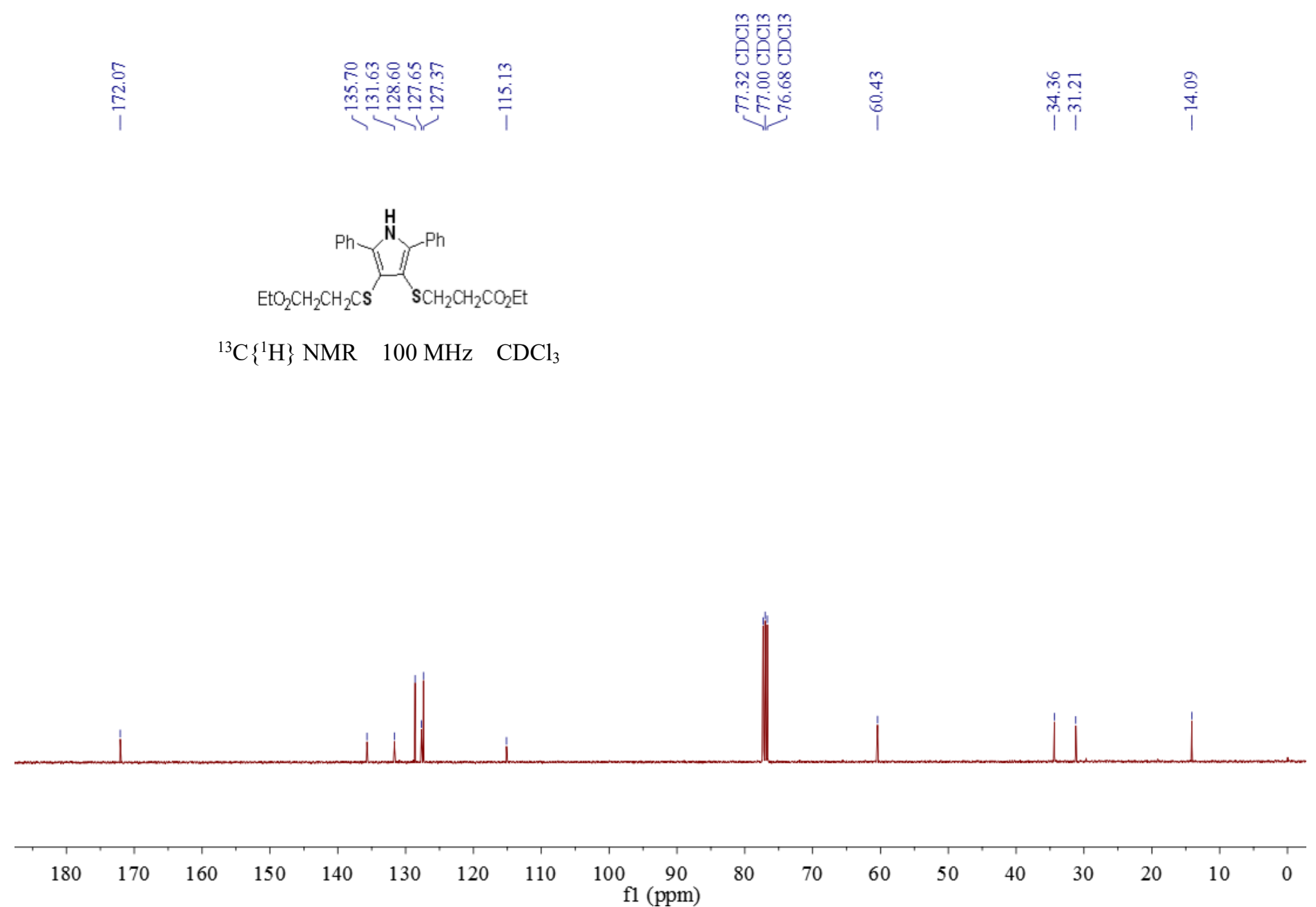
$3 m$
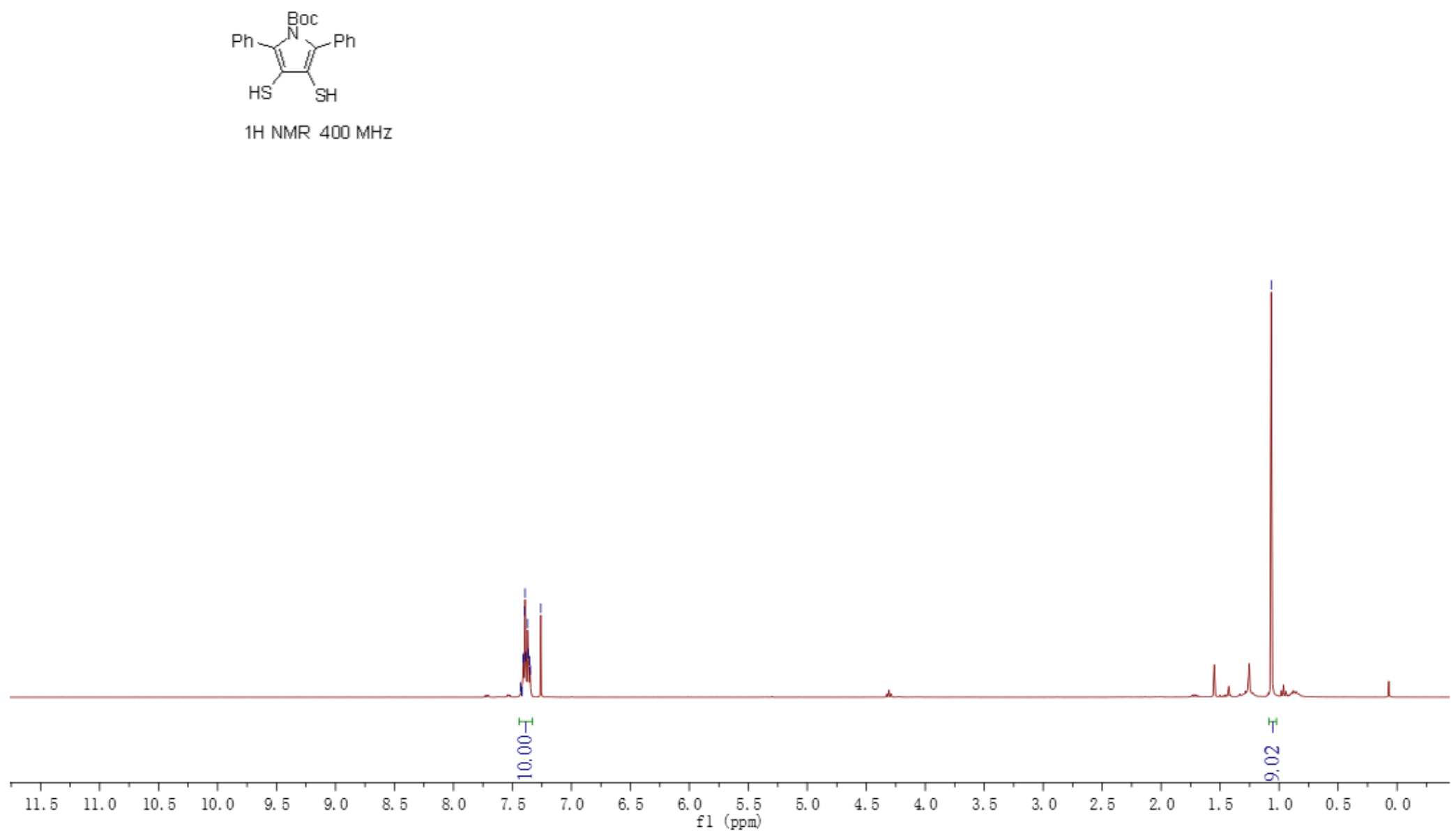


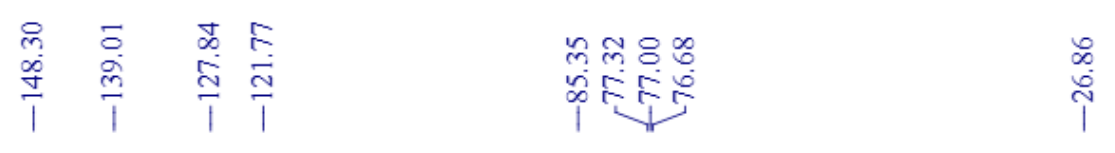

$$
\text { SS }
$$

${ }^{13} \mathrm{C}\left\{{ }^{1} \mathrm{H}\right\} \mathrm{NMR} \quad 100 \mathrm{MHz} \quad \mathrm{CDCl}_{3}$
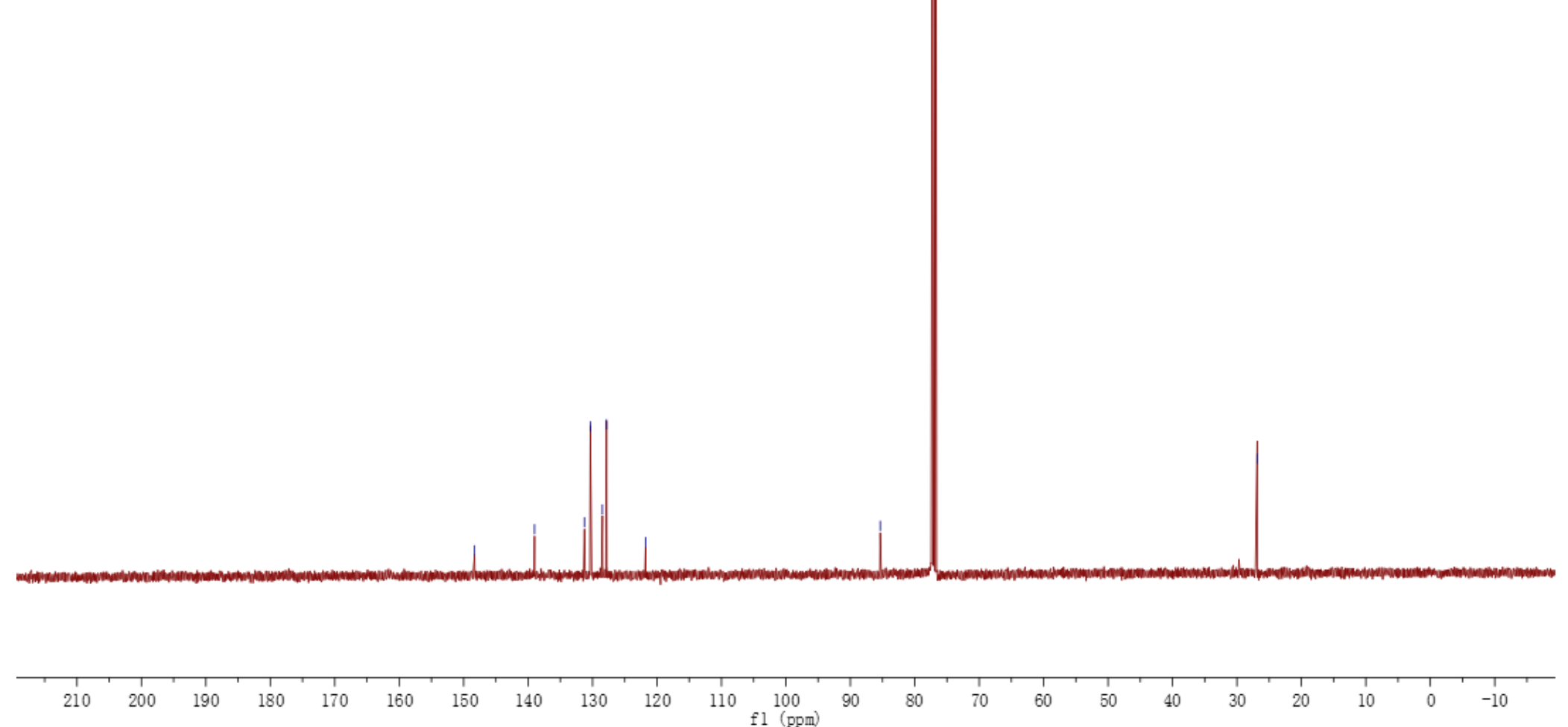


\section{$3 n$}
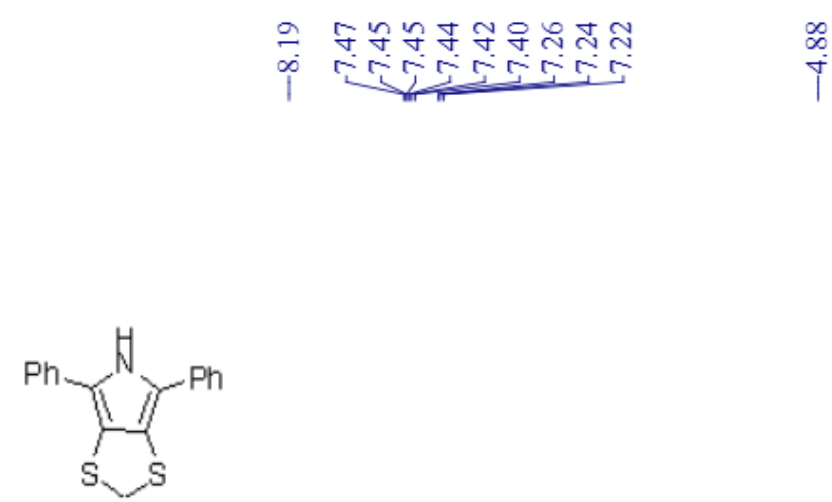

${ }^{1} \mathrm{HNMR} 400 \mathrm{MHz} \mathrm{CDCl}_{3}$

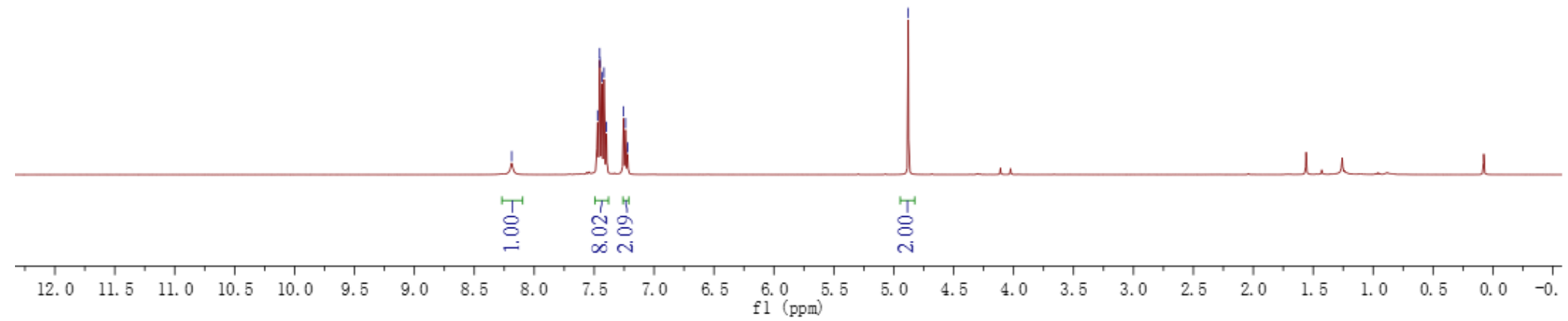


Copies of NMR spectra

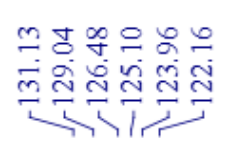

空

$\frac{1}{1}$

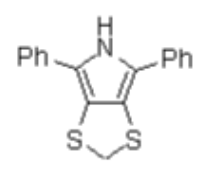

${ }^{13} \mathrm{C}\left\{{ }^{1} \mathrm{H}\right\} \mathrm{NMR} \quad 100 \mathrm{MHz} \quad \mathrm{CDCl}_{3}$

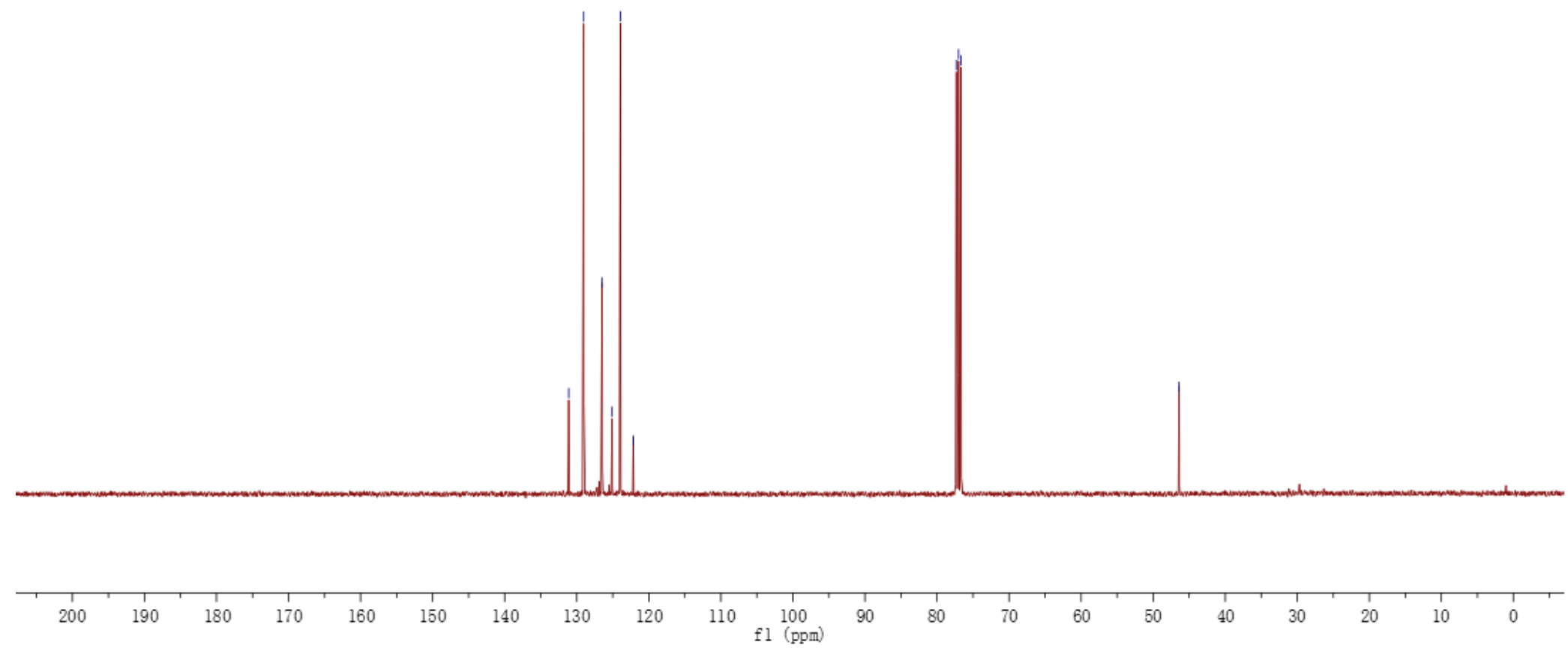

S45 
30
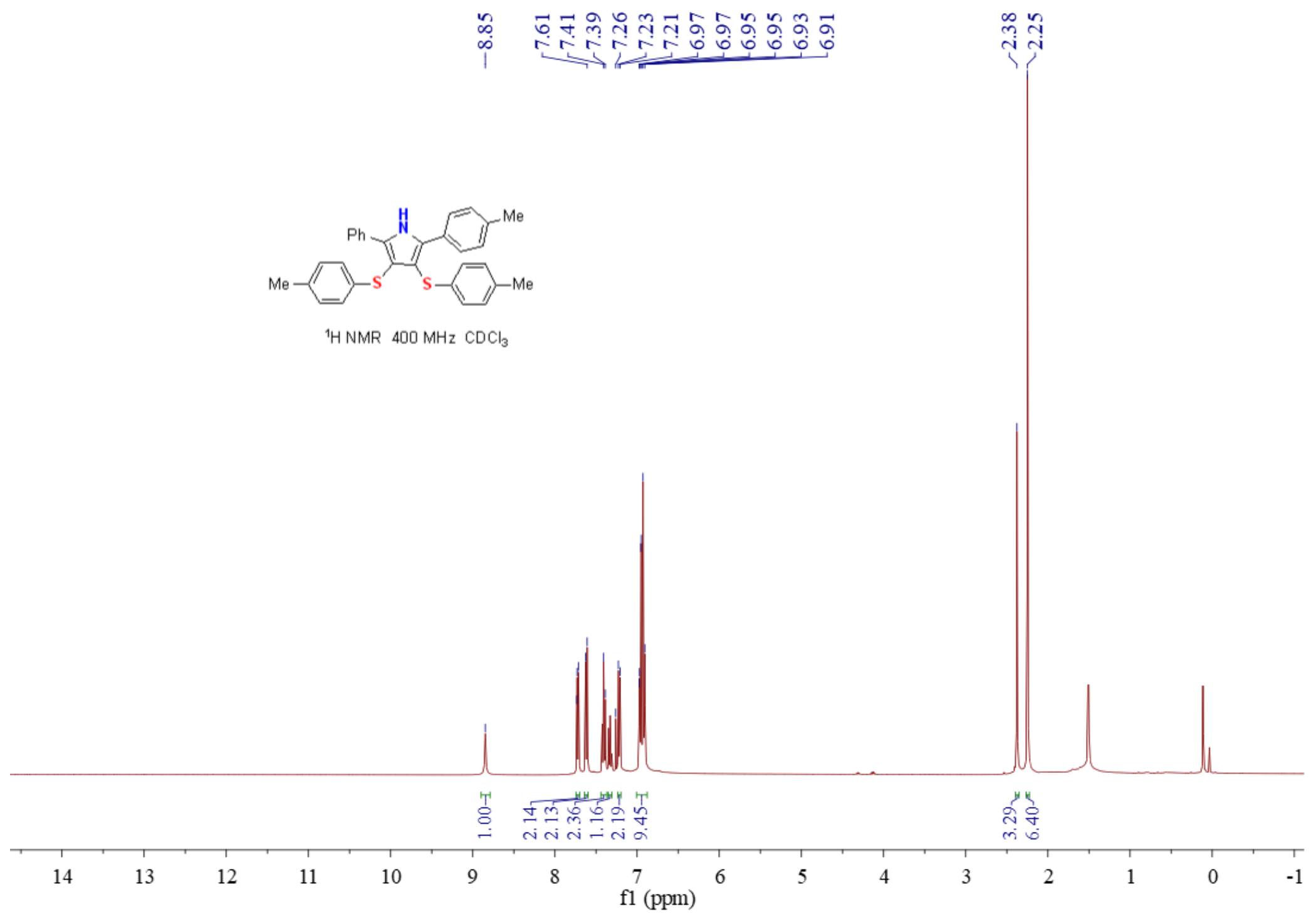


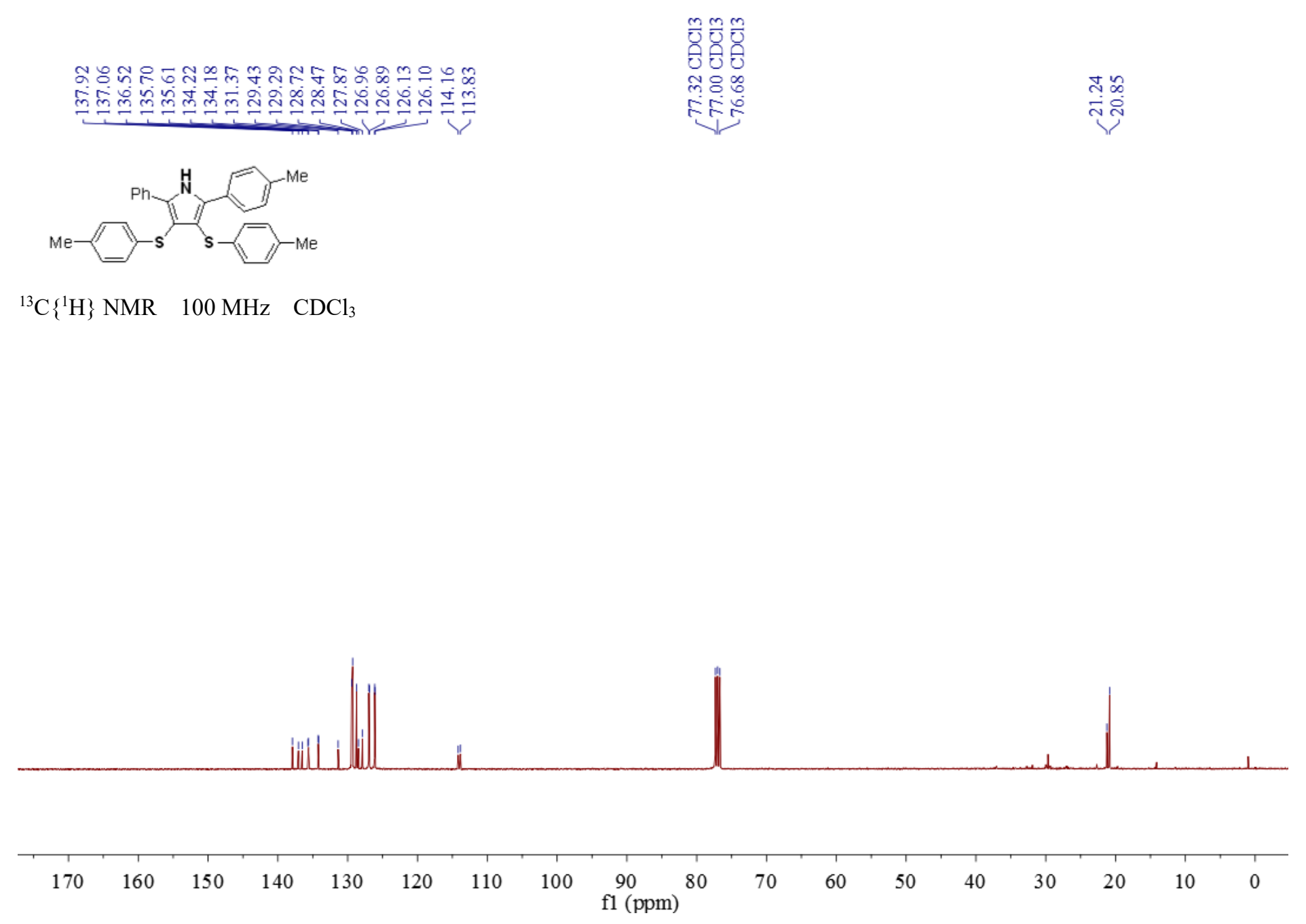




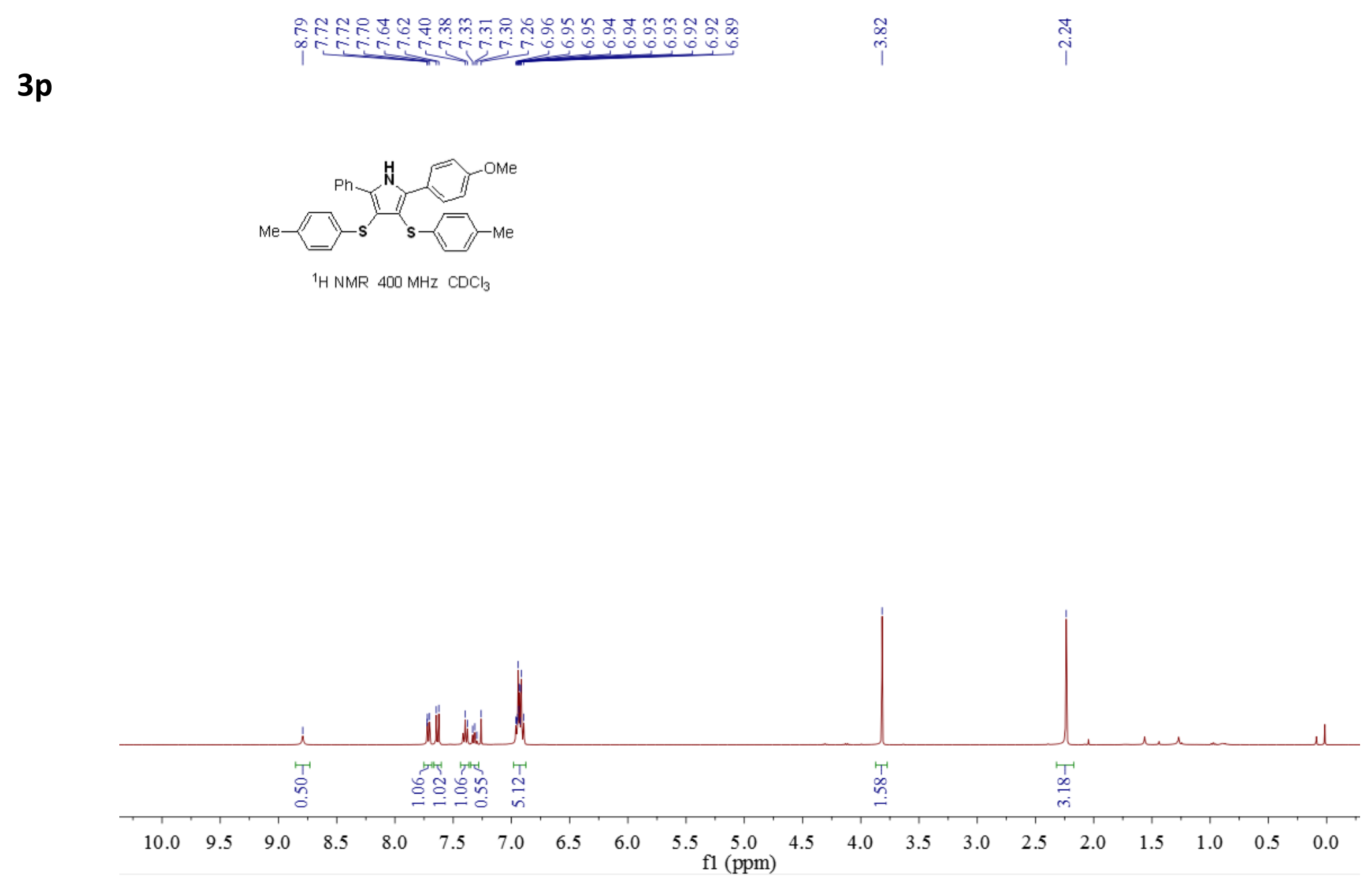




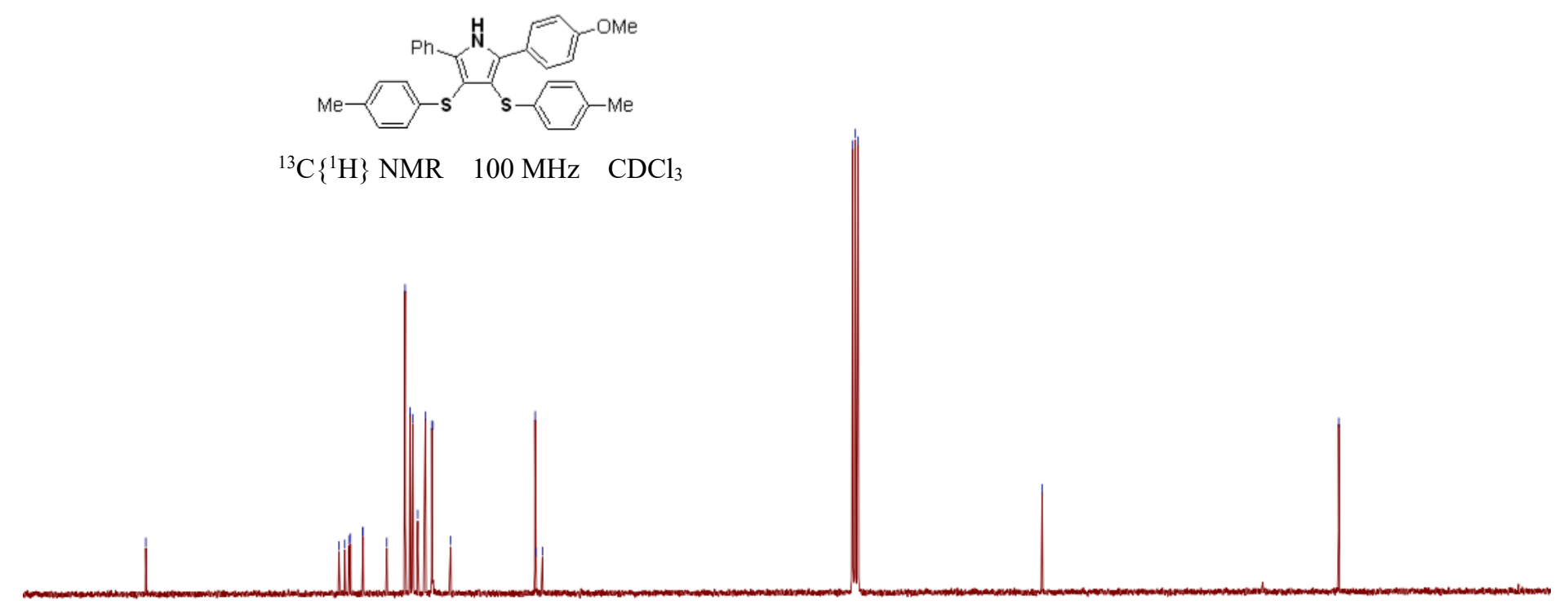


$3 q$
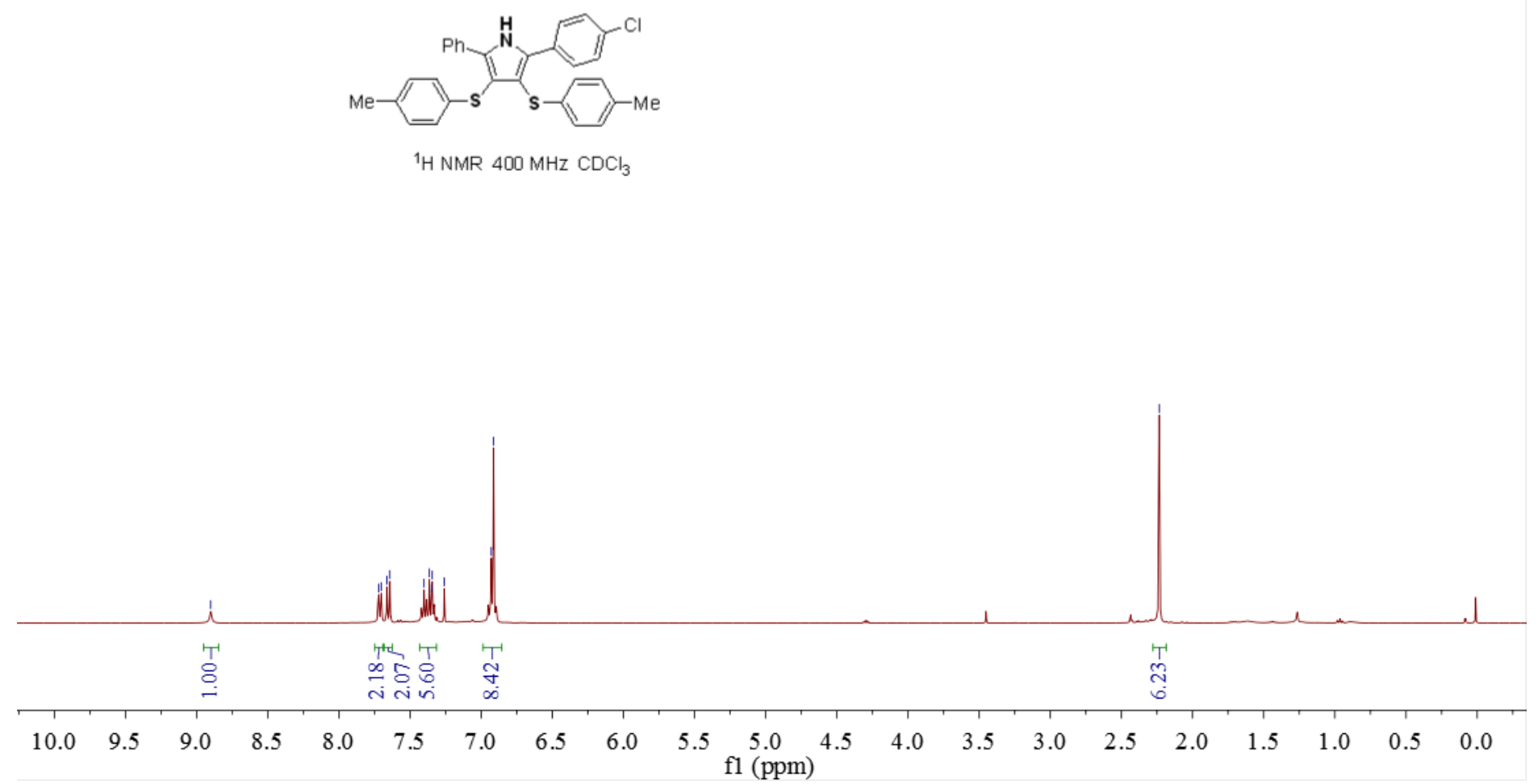
ทรติ

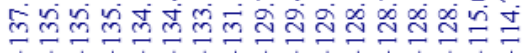

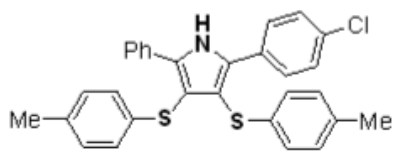

${ }^{13} \mathrm{C}\left\{{ }^{1} \mathrm{H}\right\} \mathrm{NMR} \quad 100 \mathrm{MHz} \quad \mathrm{CDCl}_{3}$

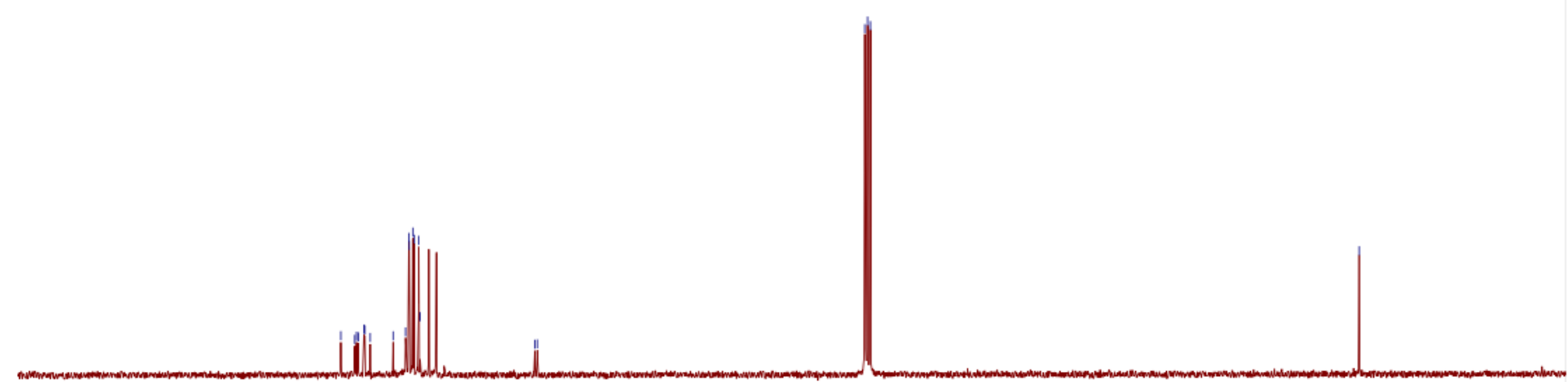

17

160

150

140

130

120

110

100

$90 \quad 80$

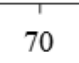

60

50

40

30

20

$10 \quad 0$ 
$3 r$
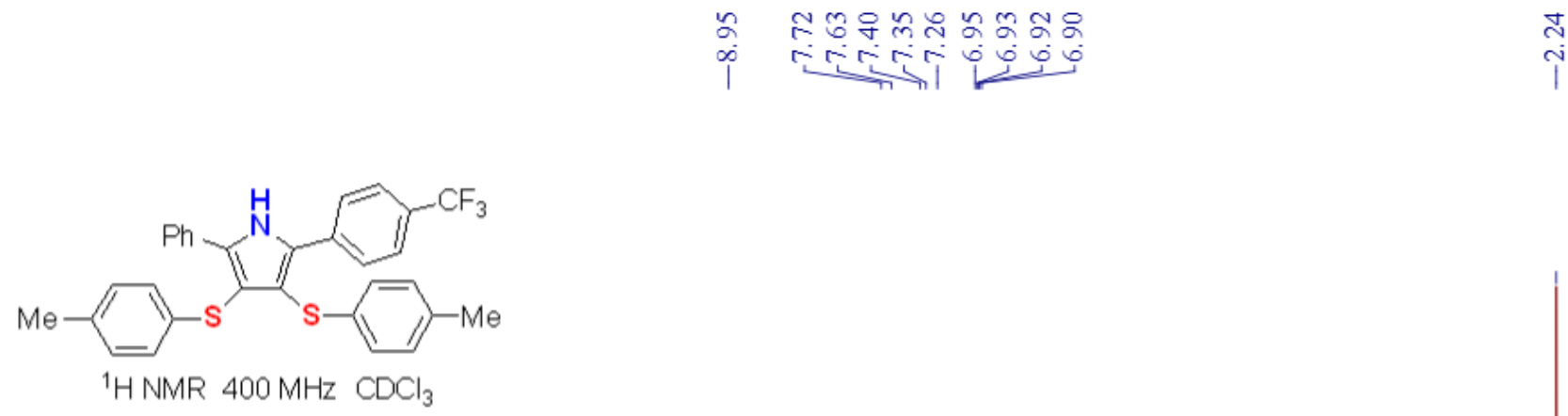

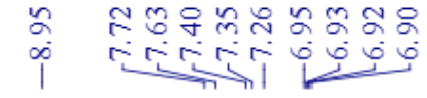

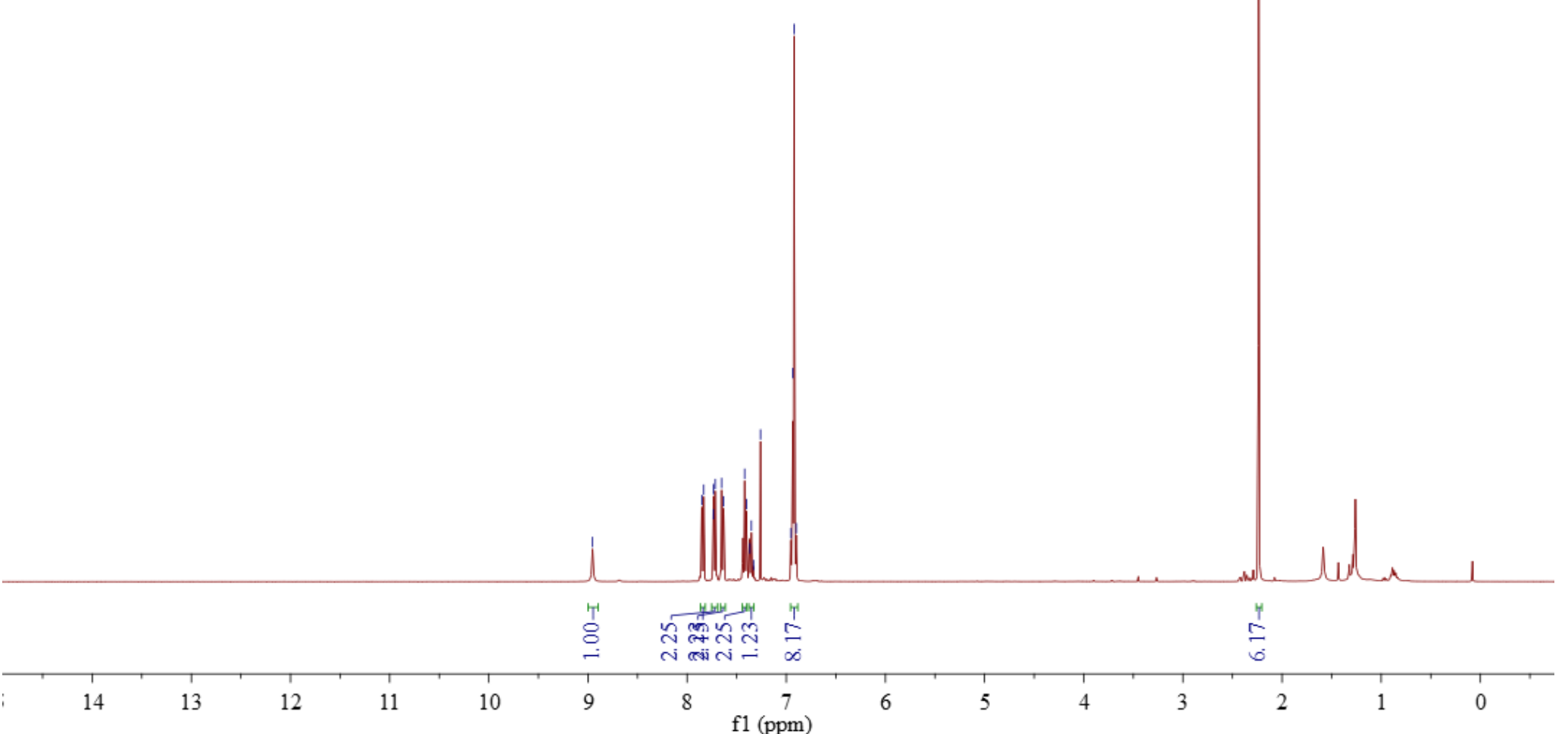




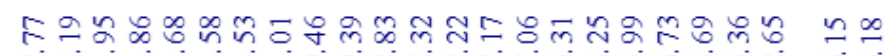

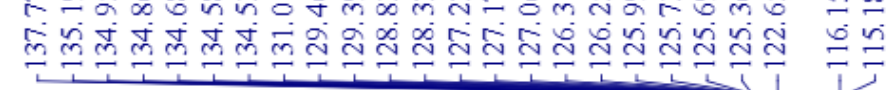

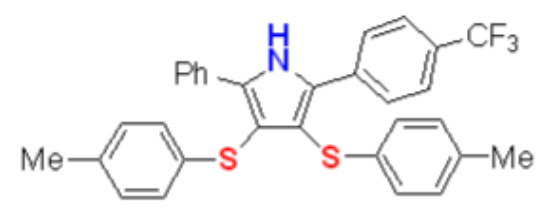

${ }^{13} \mathrm{C}\left\{{ }^{1} \mathrm{H}\right\} \mathrm{NMR} \quad 100 \mathrm{MHz} \quad \mathrm{CDCl}_{3}$

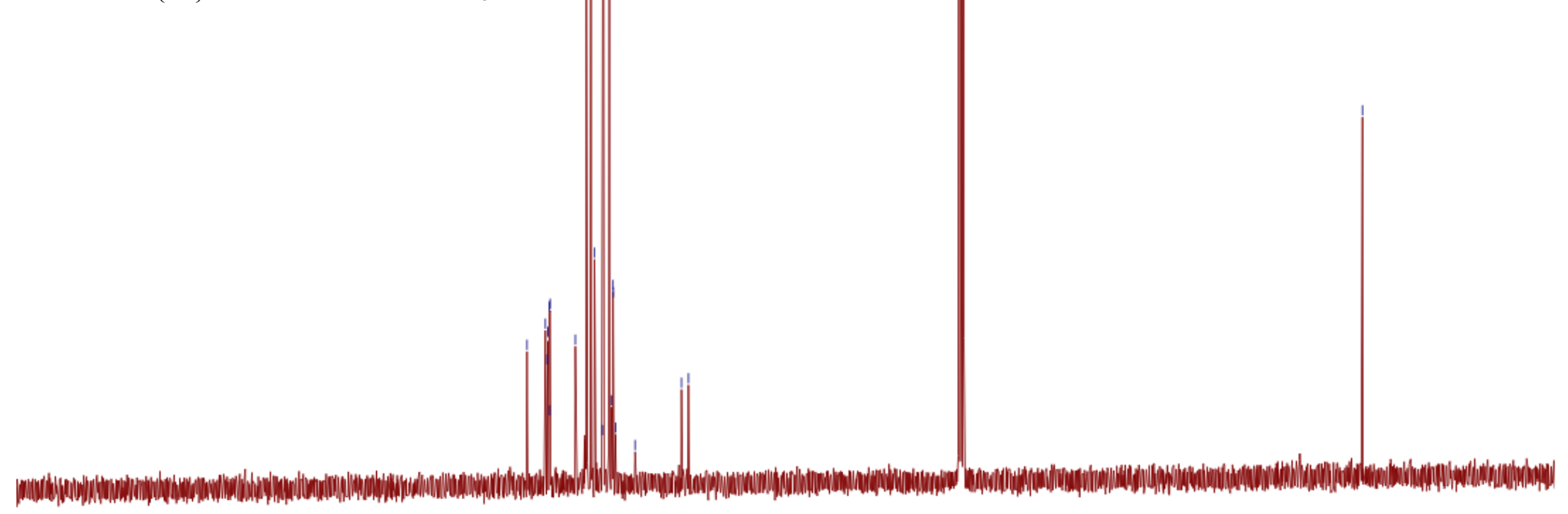



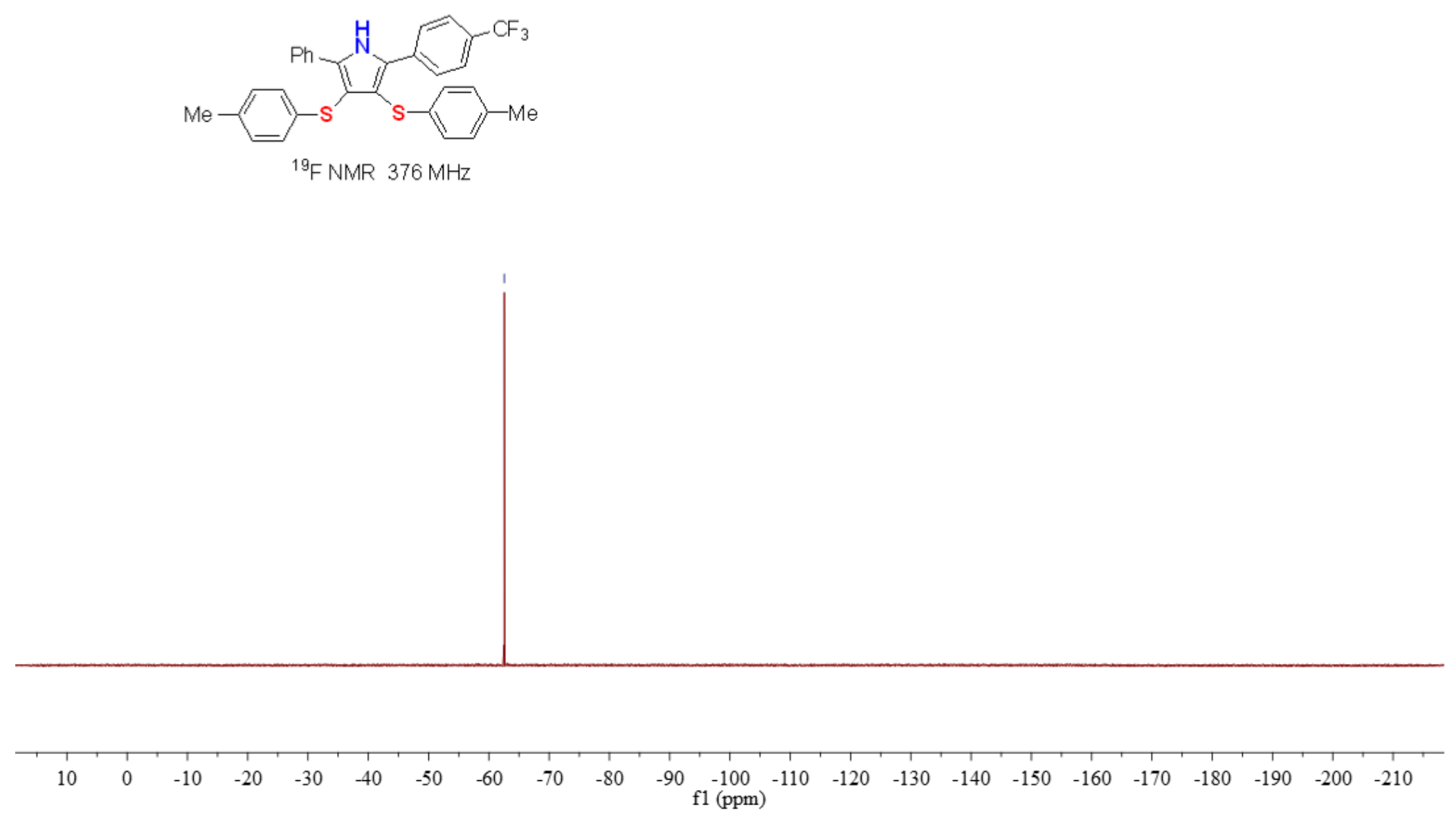


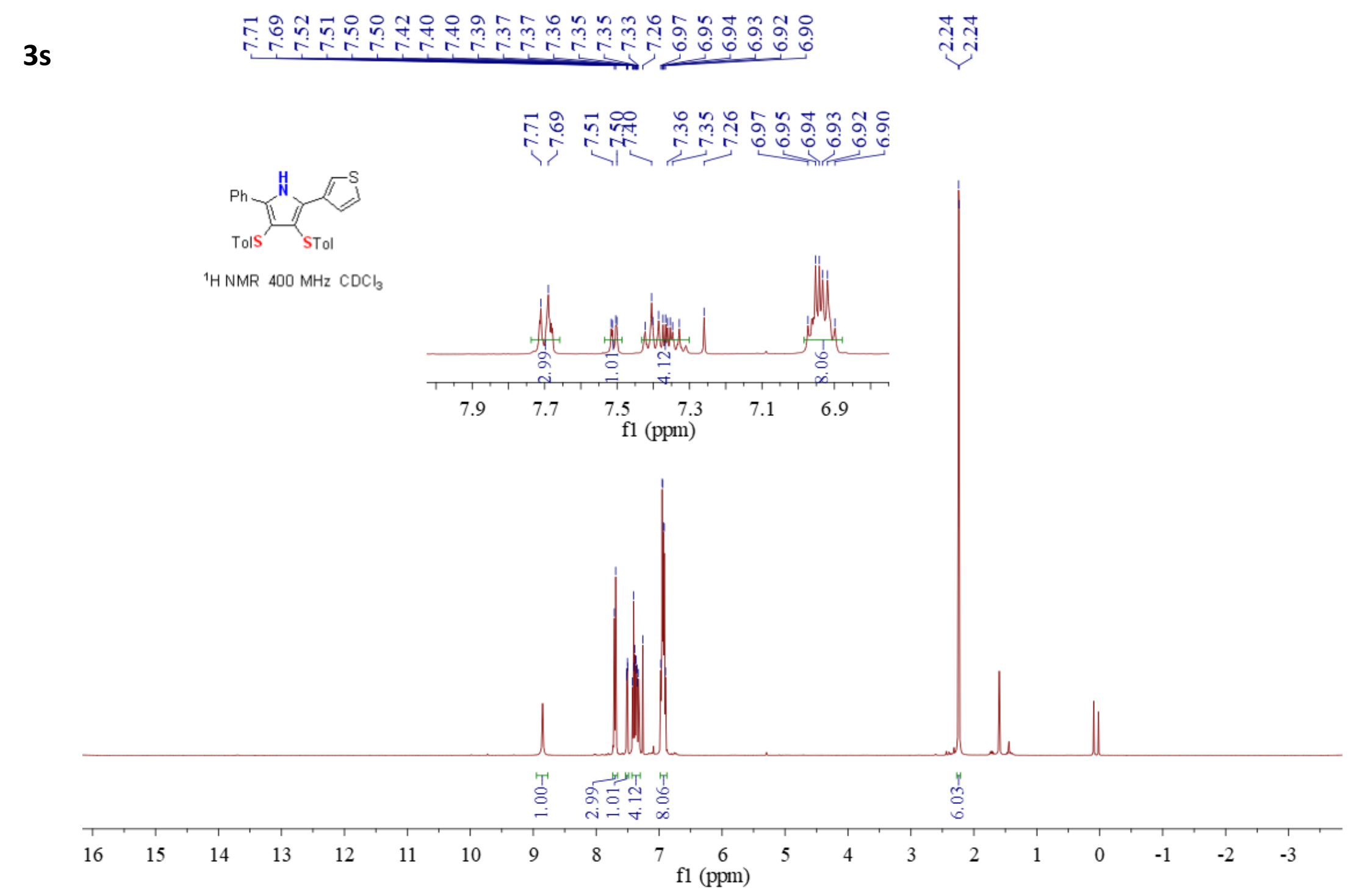


ㄴำ

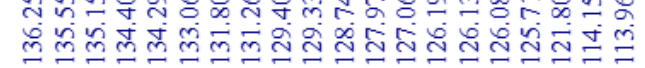

m.

m:

需

$\stackrel{\infty}{\infty}$

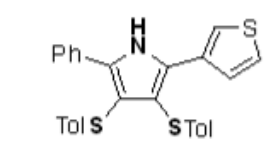

${ }^{13} \mathrm{C}\left\{{ }^{1} \mathrm{H}\right\} \mathrm{NMR} \quad 100 \mathrm{MHz} \quad \mathrm{CDCl}_{3}$

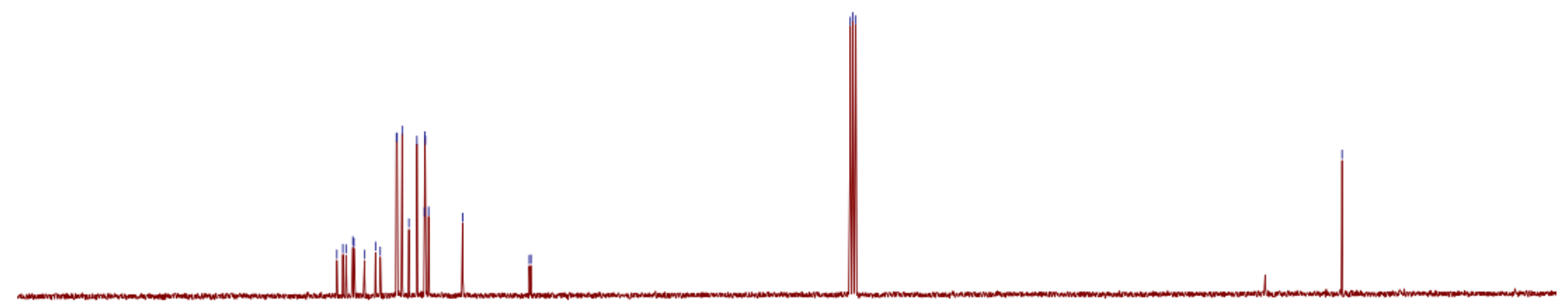

$170 \quad 160$

$160 \quad 150$

140

130

120

110

$90 \quad 80$

50

40

30

20

$1 0 \longdiv { 1 }$

(ppm) 


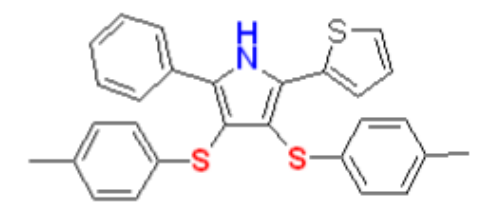

${ }^{1} \mathrm{HNMR} 400 \mathrm{MHz} \mathrm{CDCl}_{3}$

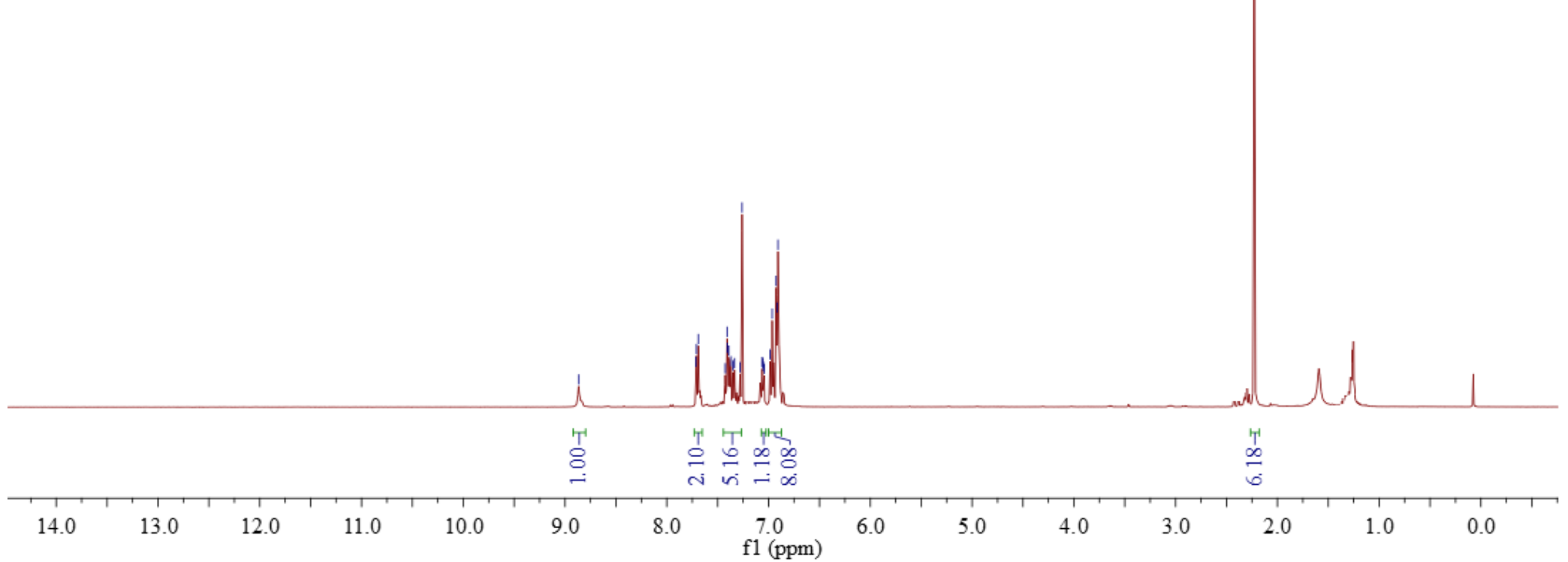




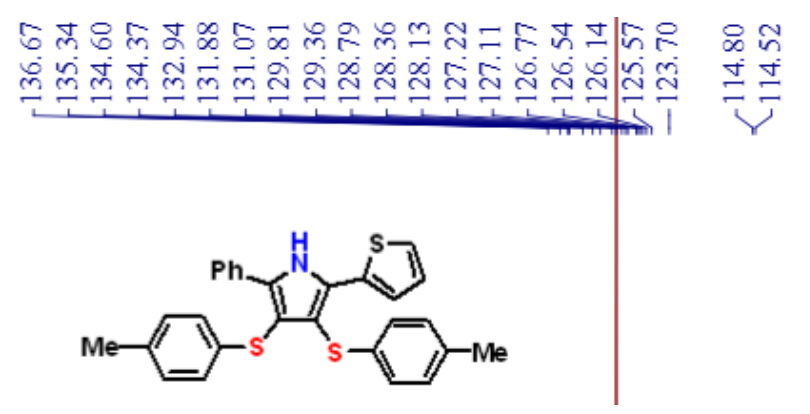

${ }^{13} \mathrm{C}\left\{{ }^{1} \mathrm{H}\right\} \mathrm{NMR} \quad 100 \mathrm{MHz} \quad \mathrm{CDCl}_{3}$

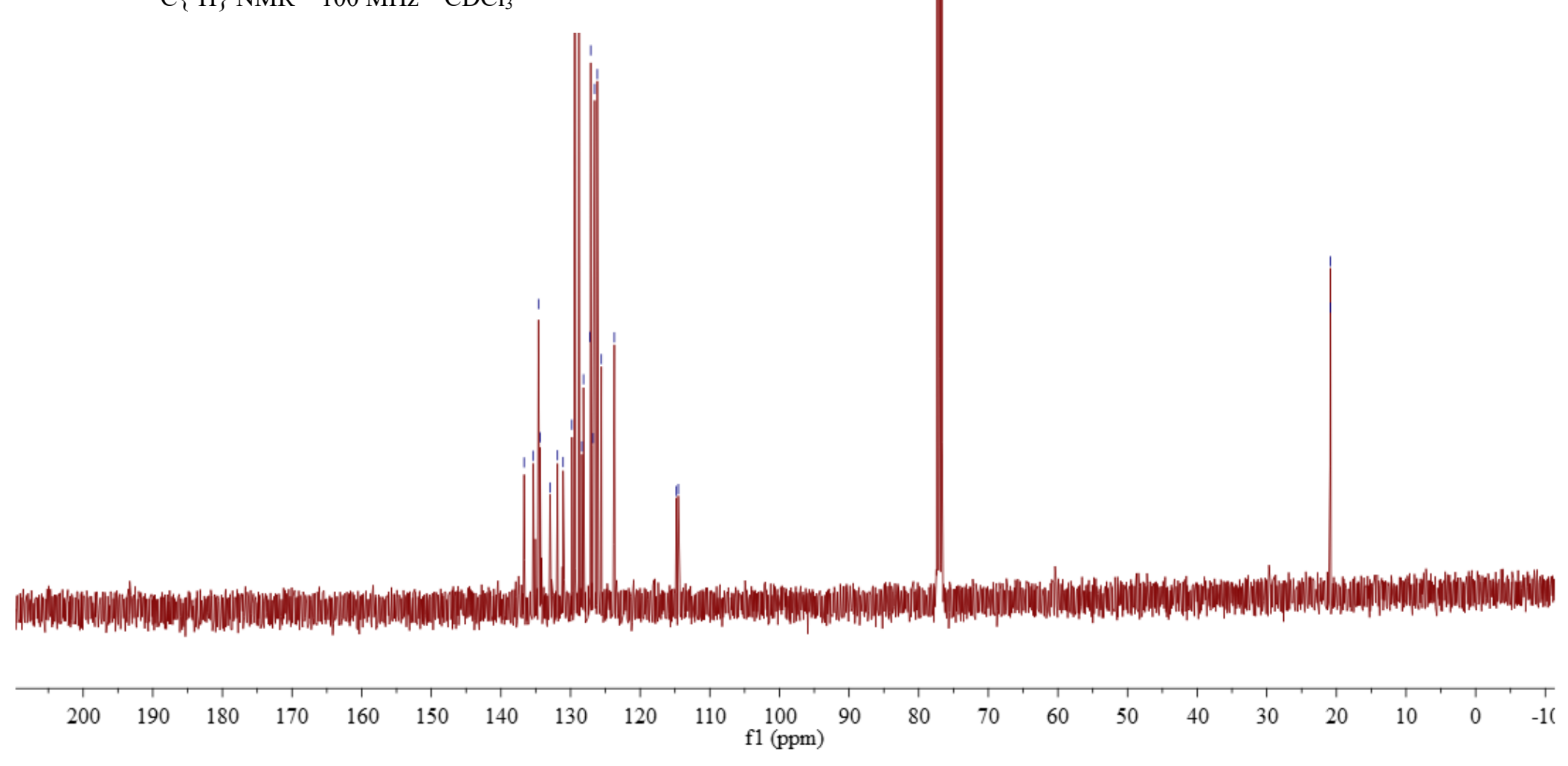


3u

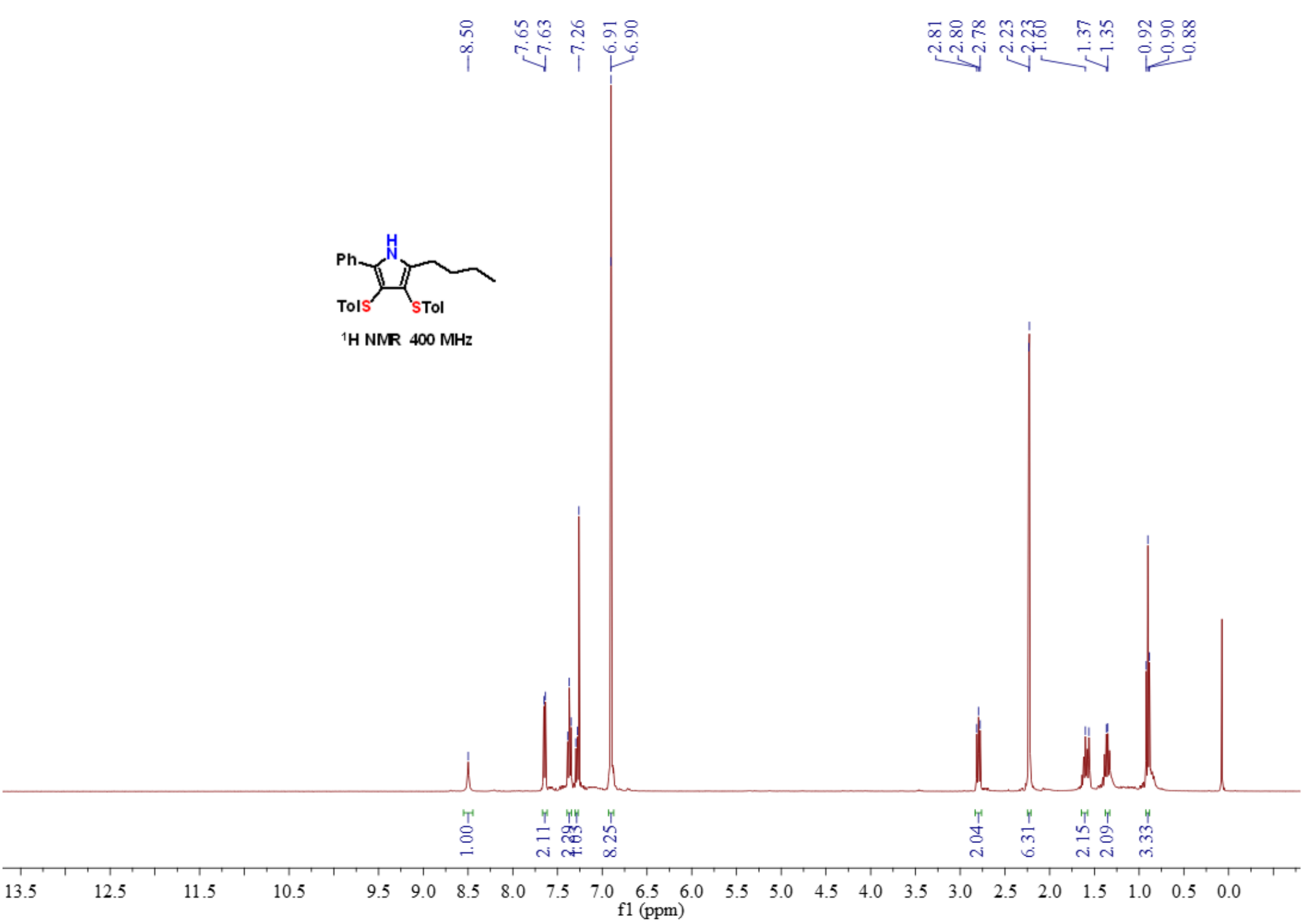



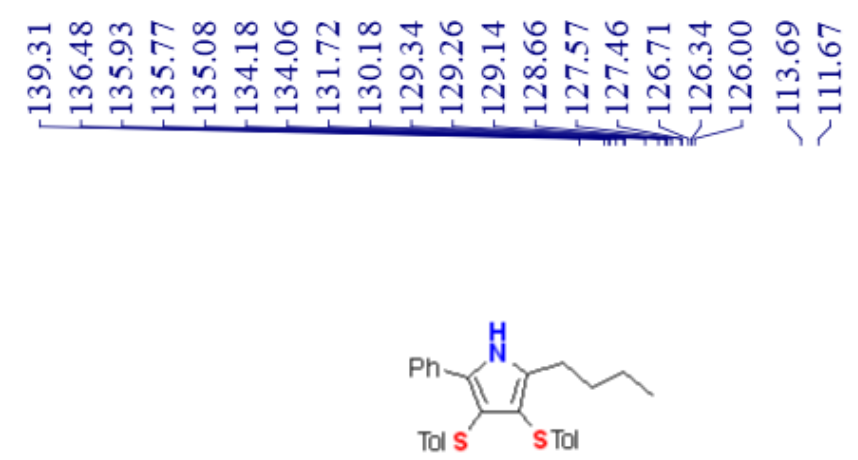

${ }^{13} \mathrm{C}\left\{{ }^{1} \mathrm{H}\right\} \mathrm{NMR} \quad 100 \mathrm{MHz} \quad \mathrm{CDCl}_{3}$

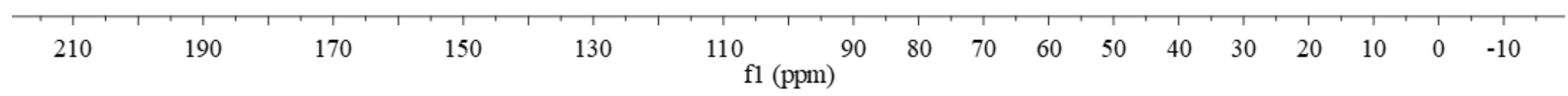


3v

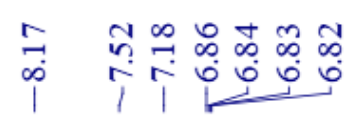

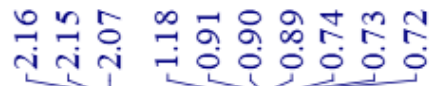

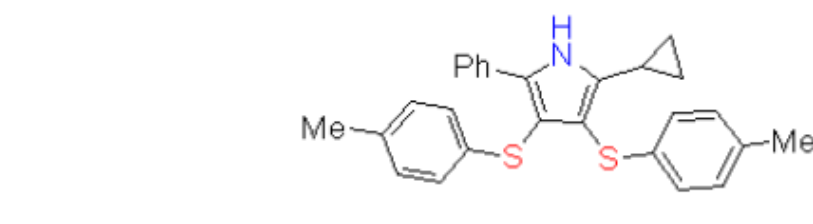

${ }^{1} \mathrm{HNMR} 400 \mathrm{MHz} \mathrm{CDCl}_{3}$

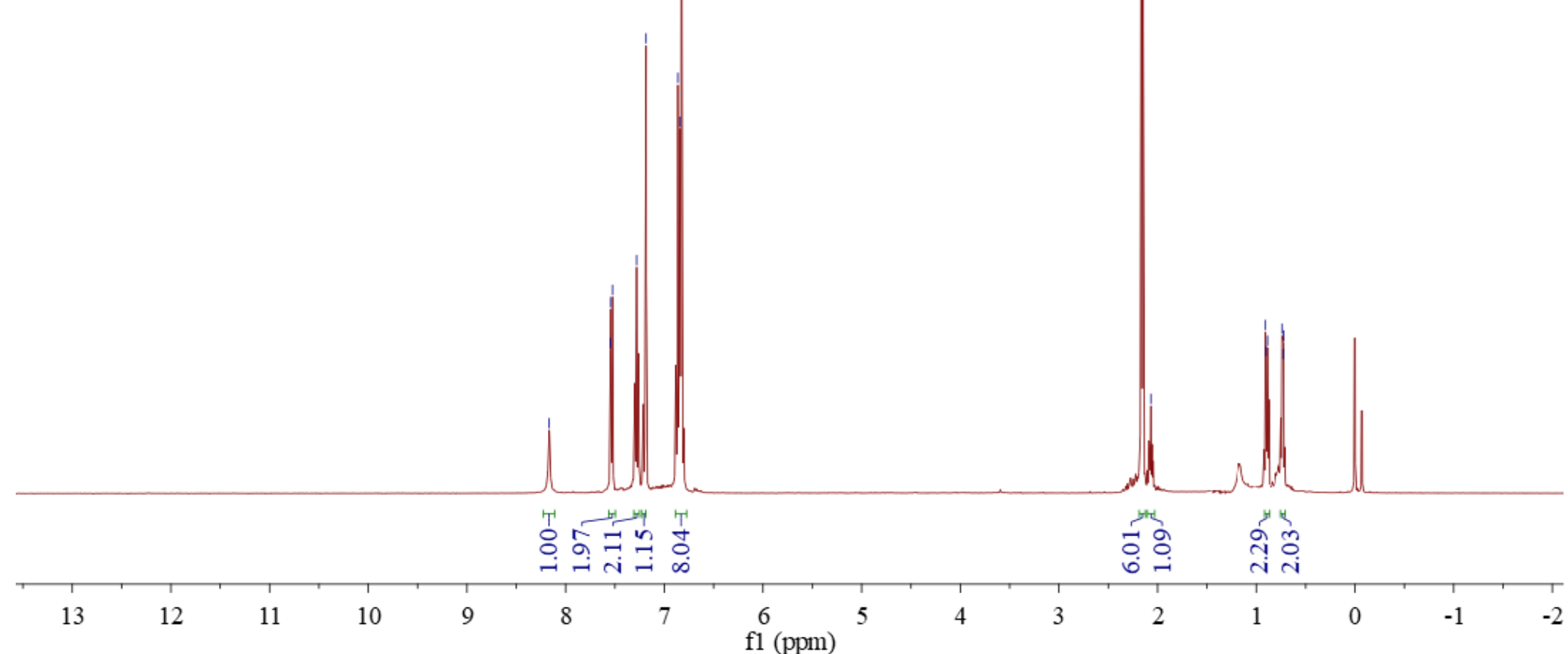




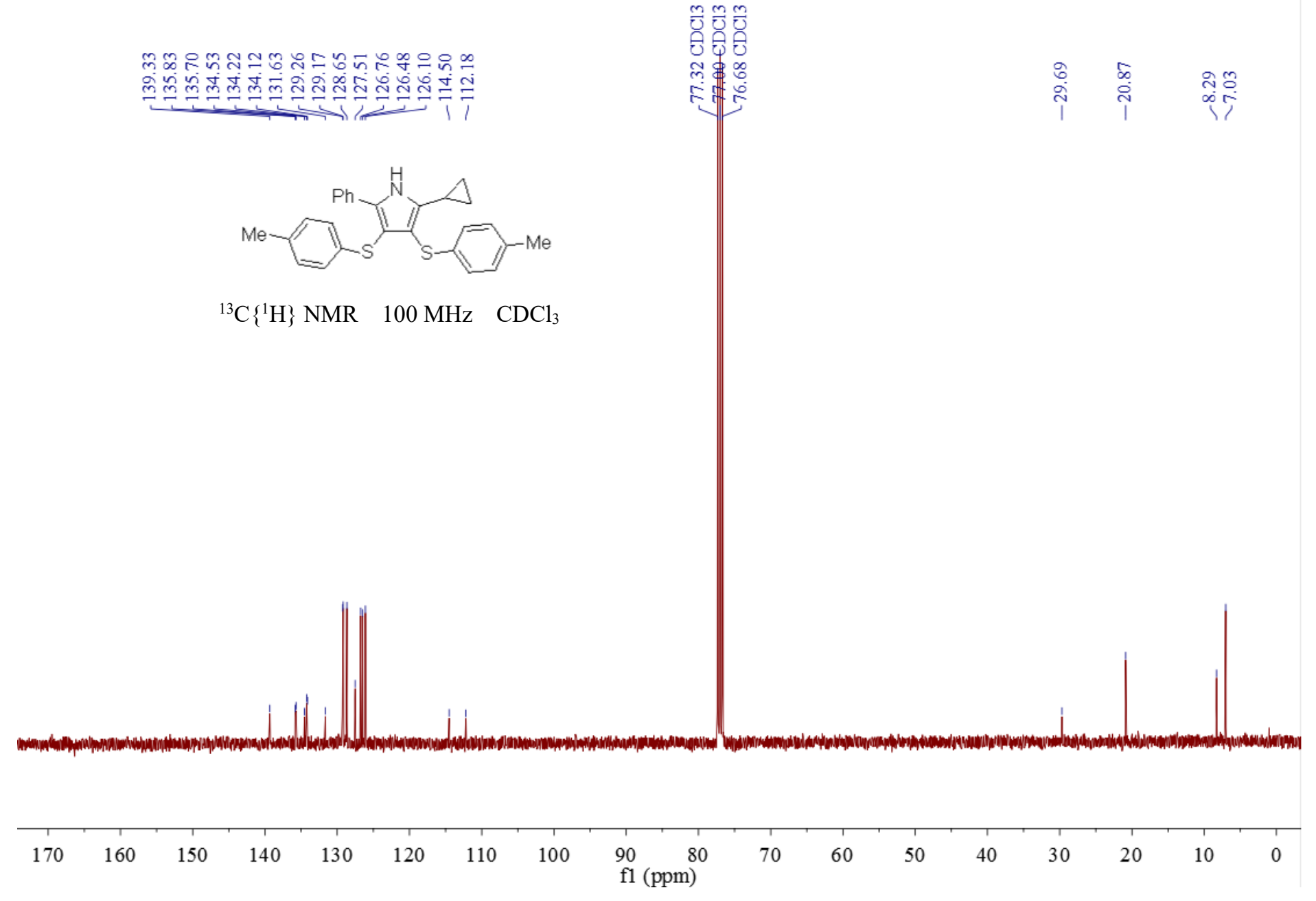




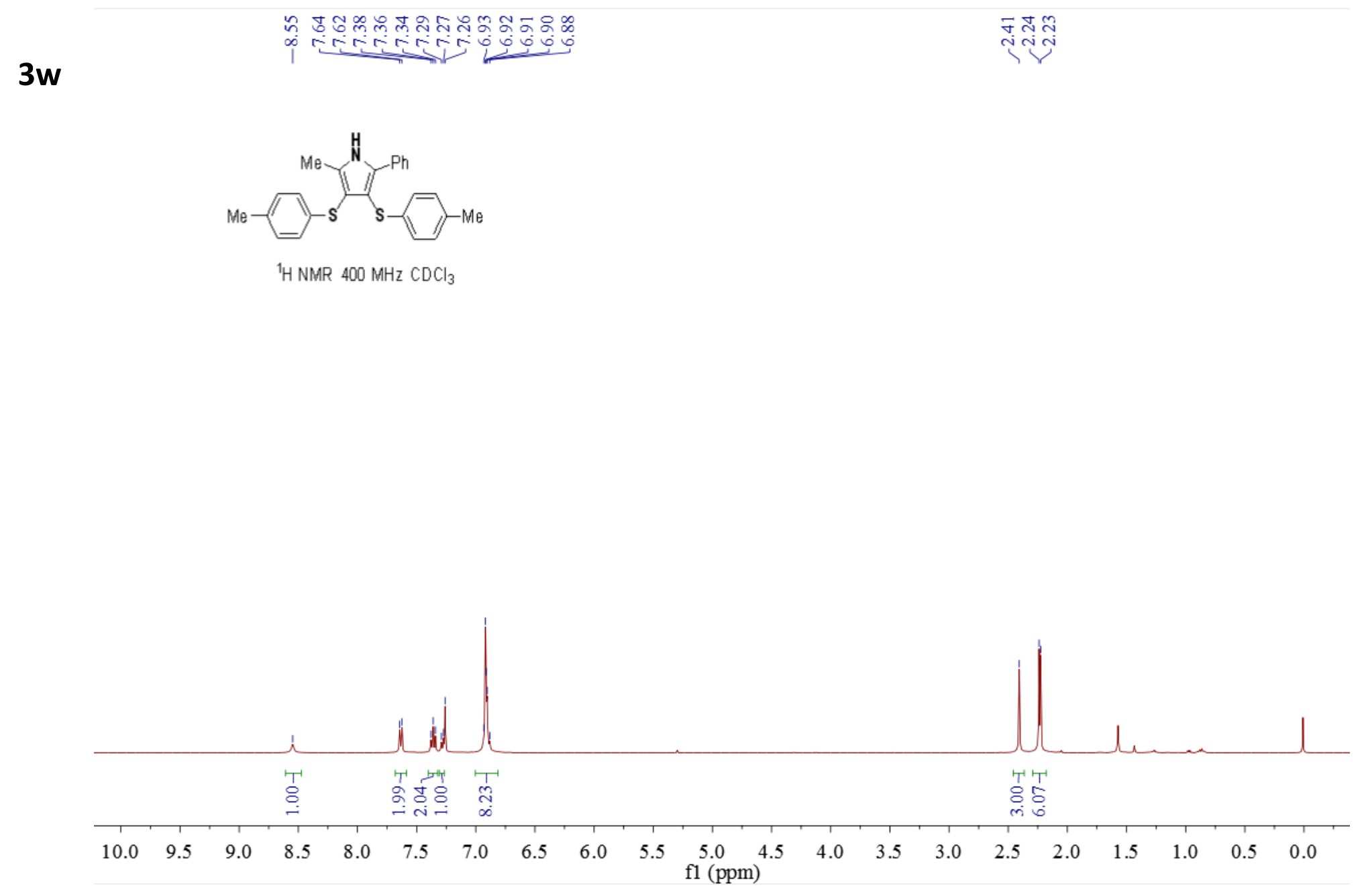




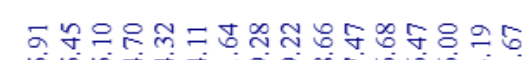

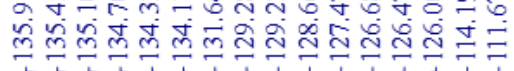

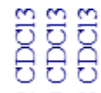

No

范

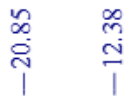

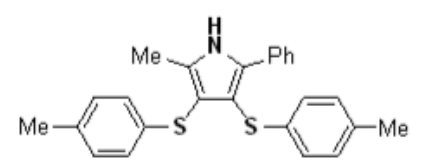

${ }^{13} \mathrm{C}\left\{{ }^{1} \mathrm{H}\right\}$ NMR $100 \mathrm{MHz} \quad \mathrm{CDCl}_{3}$

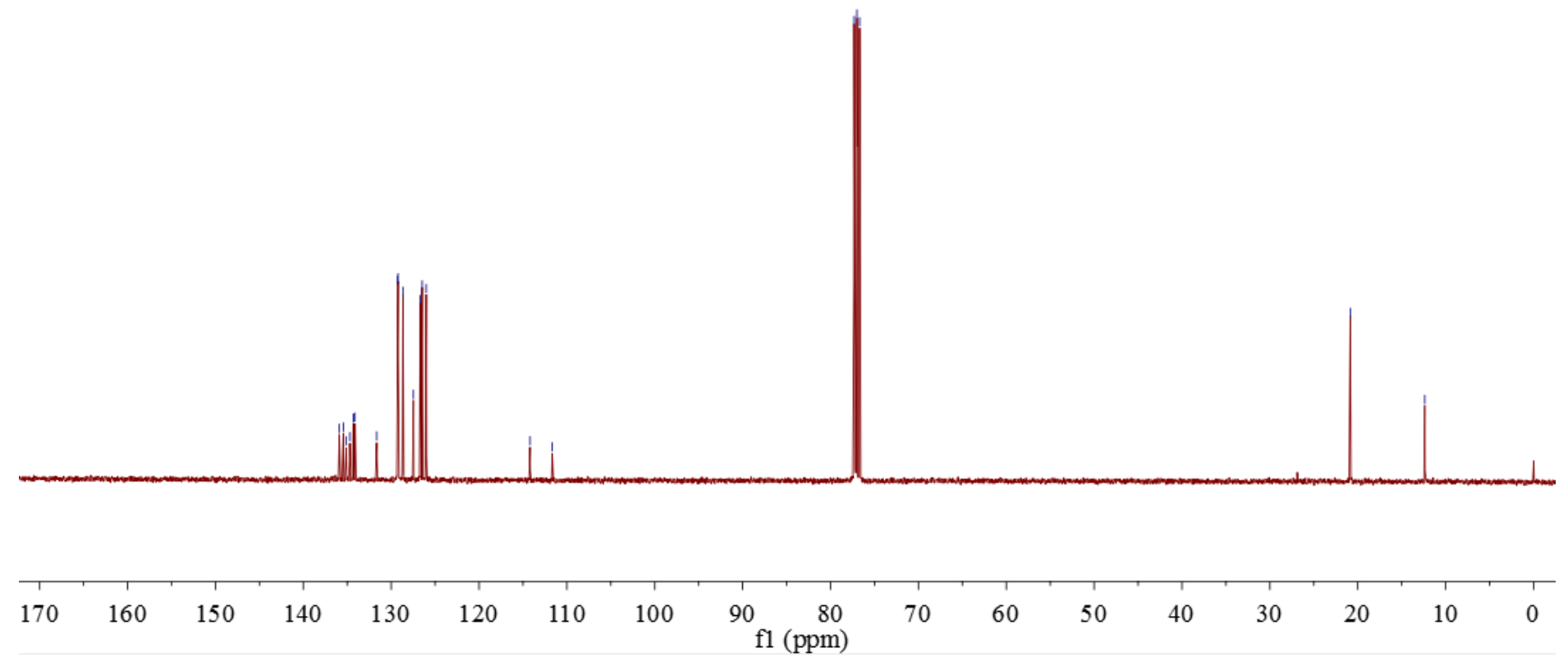



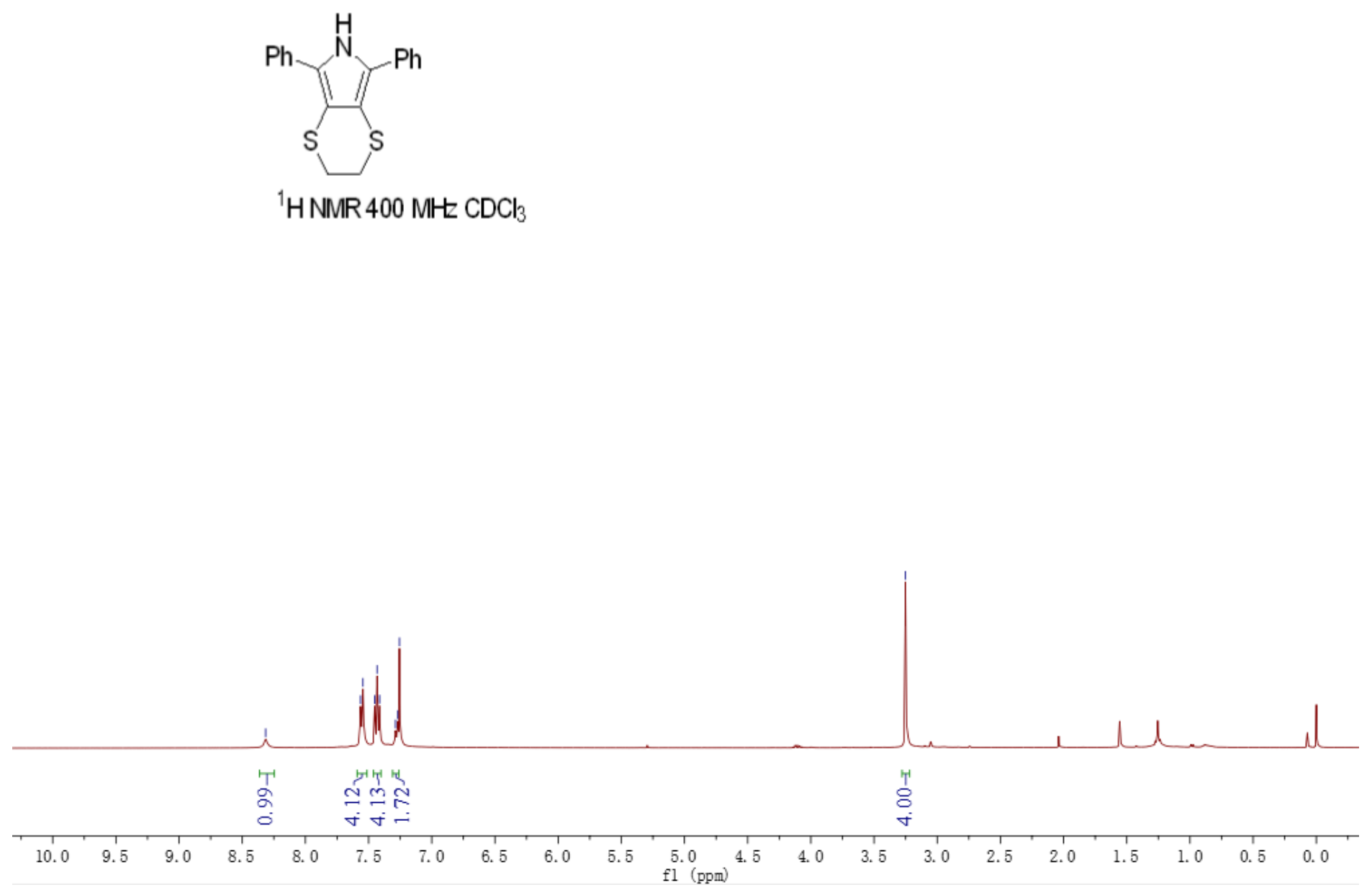

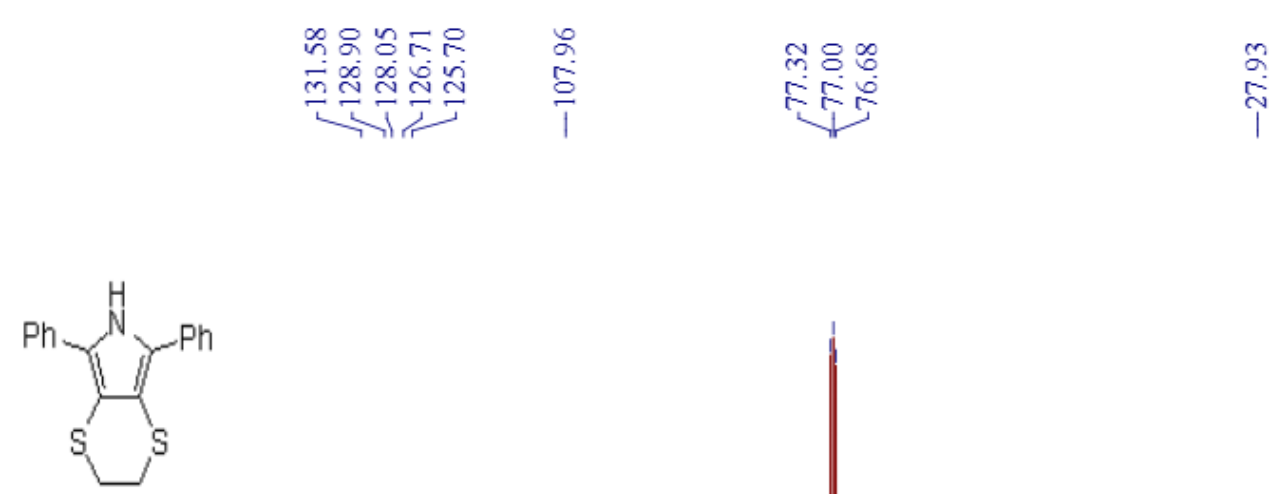

${ }^{13} \mathrm{C}\left\{{ }^{1} \mathrm{H}\right\} \mathrm{NMR} \quad 100 \mathrm{MHz} \quad \mathrm{CDCl}_{3}$
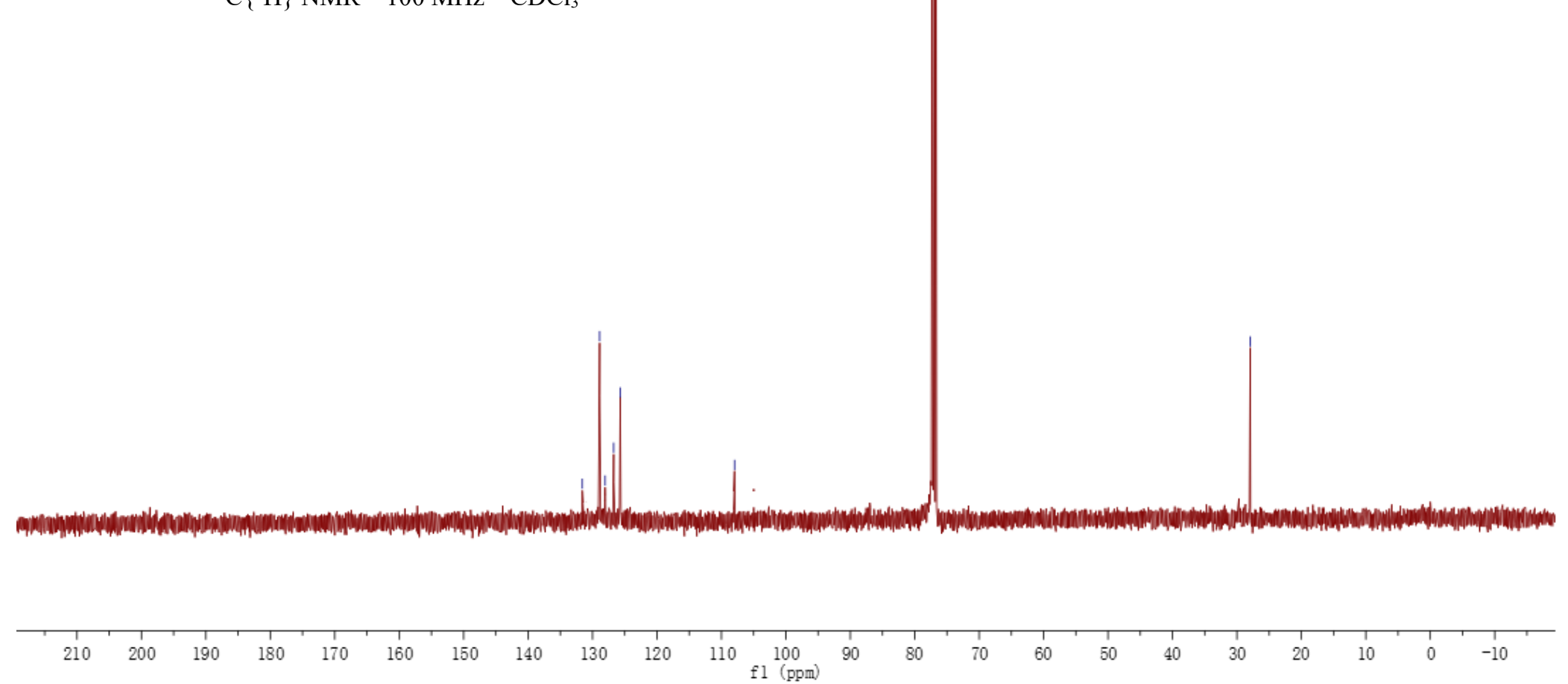


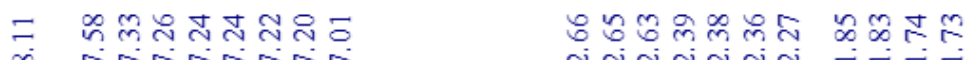

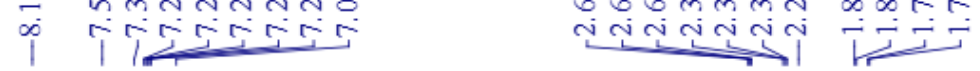

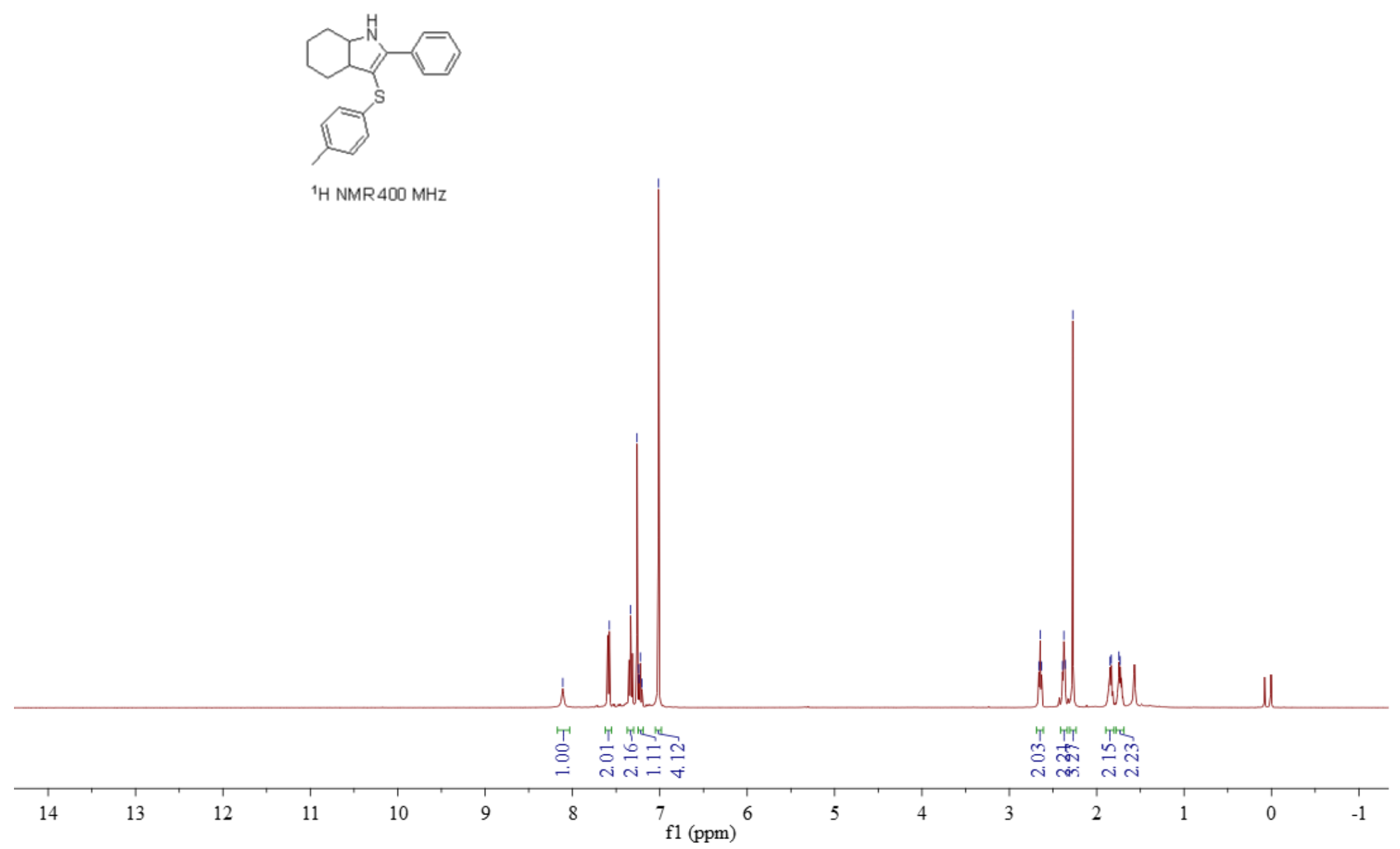




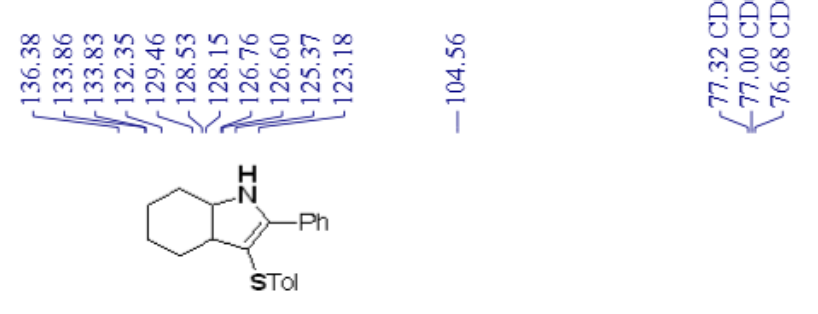

ำ

กูกู่สูก

${ }^{13} \mathrm{C}\left\{{ }^{1} \mathrm{H}\right\} \mathrm{NMR} \quad 100 \mathrm{MHz} \quad \mathrm{CDCl}_{3}$

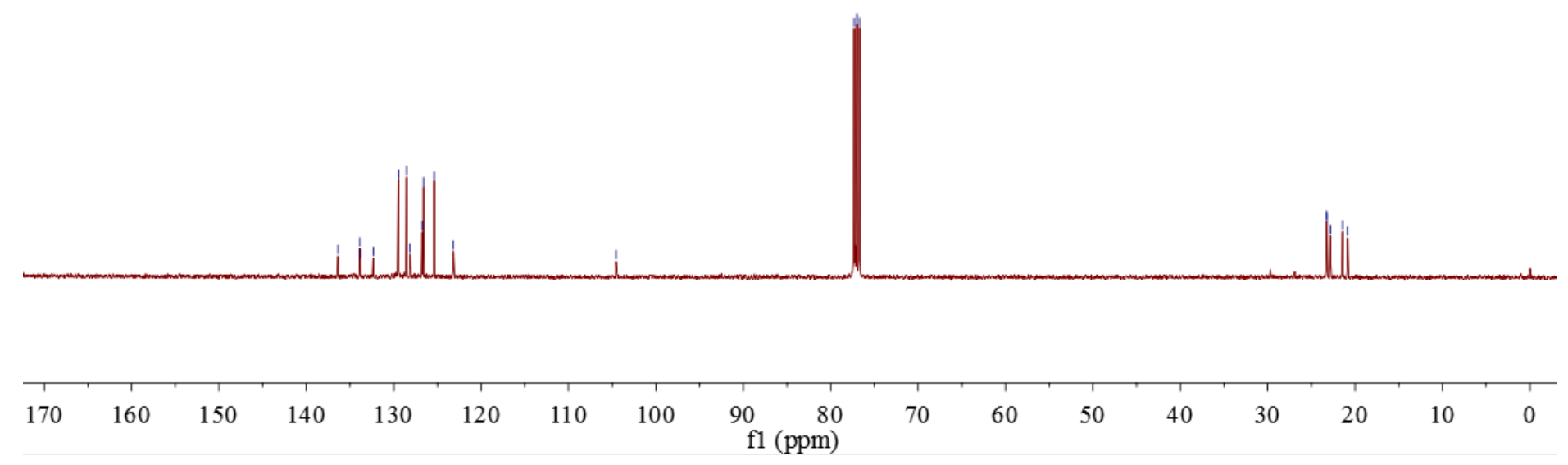


$3 z$

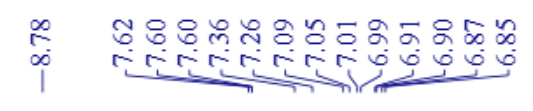

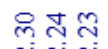

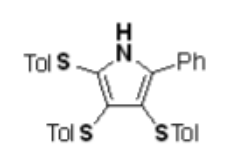

${ }^{1} \mathrm{H} \mathrm{NMR} 400 \mathrm{MHz} \mathrm{CDCl}_{3}$

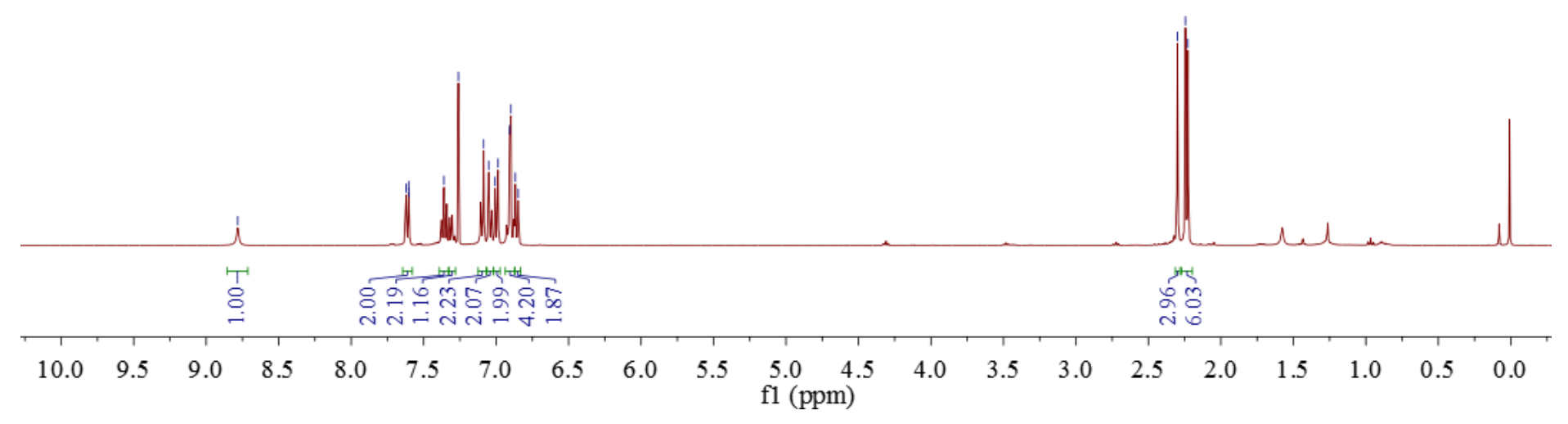




$$
\text { Tols }{ }_{\text {Tols }}^{H} \int_{\text {STO }}^{\mathrm{Ph}}
$$

${ }^{13} \mathrm{C}\left\{{ }^{1} \mathrm{H}\right\}$ NMR $100 \mathrm{MHz} \quad \mathrm{CDCl}_{3}$

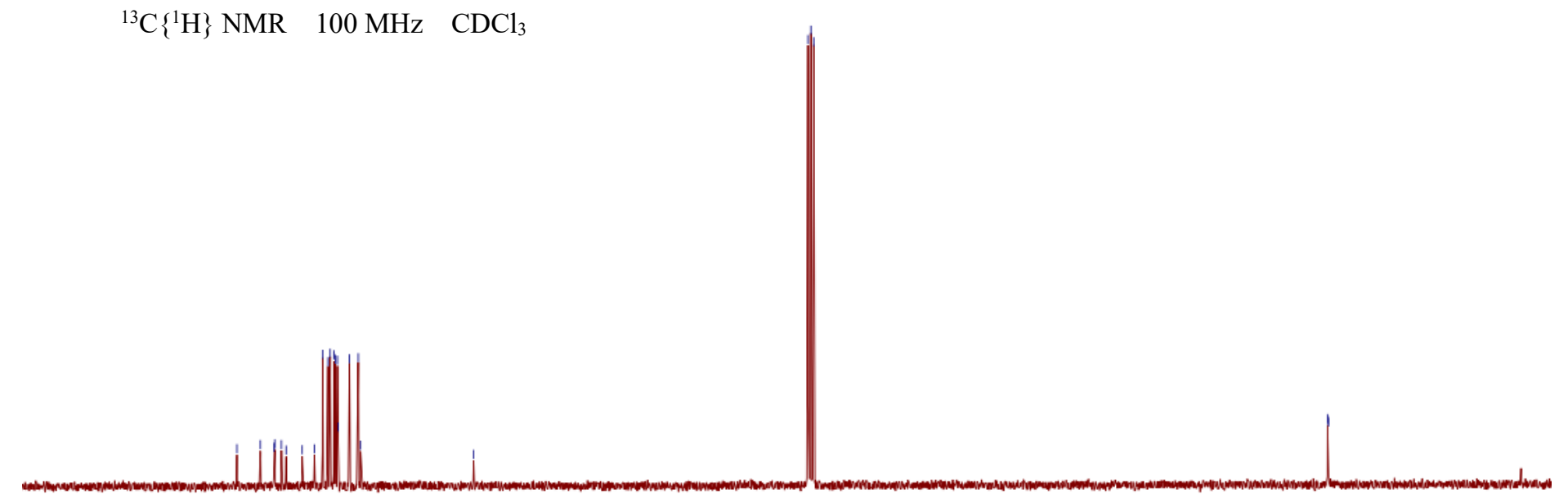


2,5-Diphenyl-3,4-bis(p-tolylthio)-1H-pyrrole (3a)

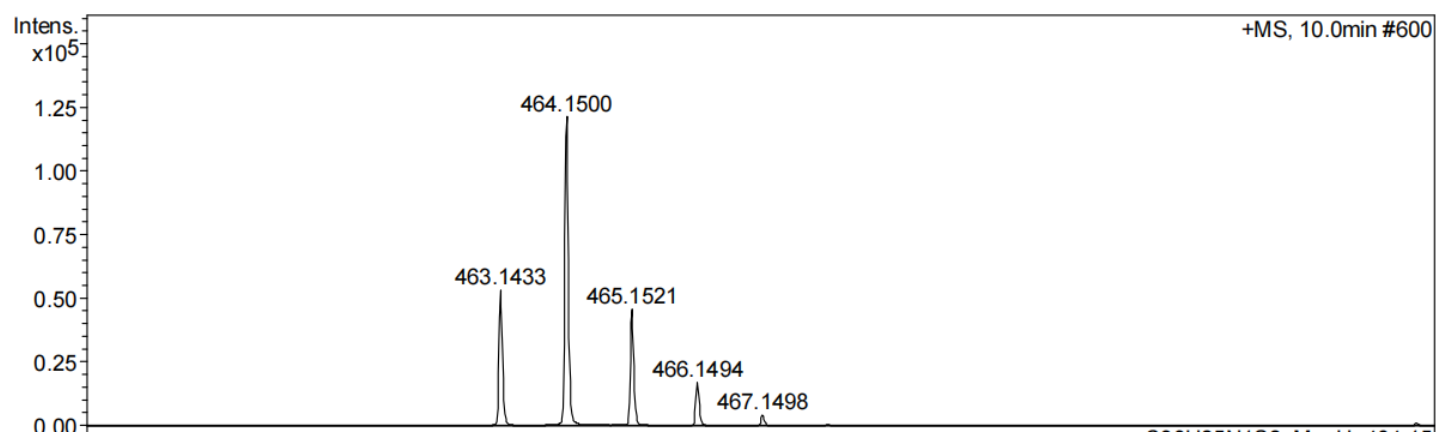

2,5-Diphenyl-3,4-bis(phenylthio)-1H-pyrrole (3b)

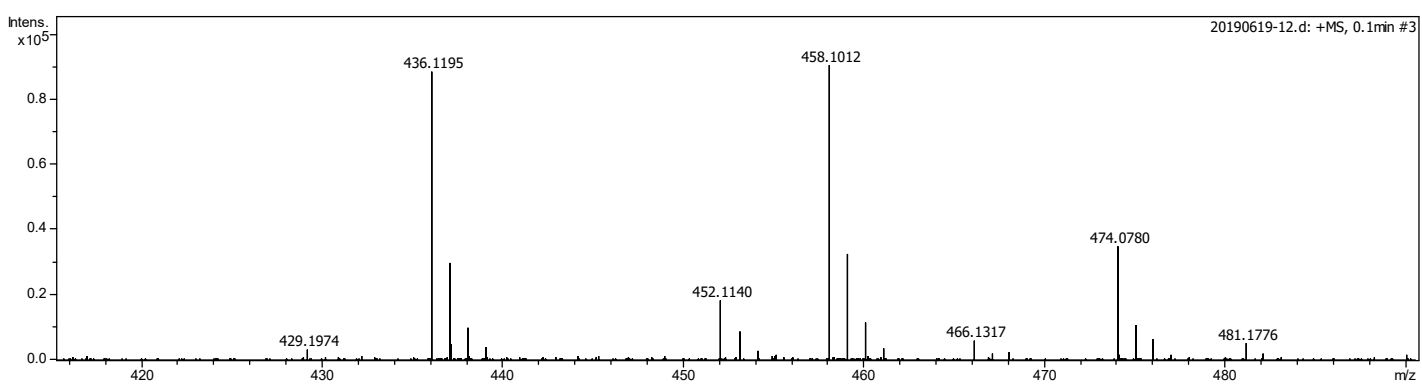

3,4-Bis((4-methoxyphenyl)thio)-2,5-diphenyl-1H-pyrrole (3c)

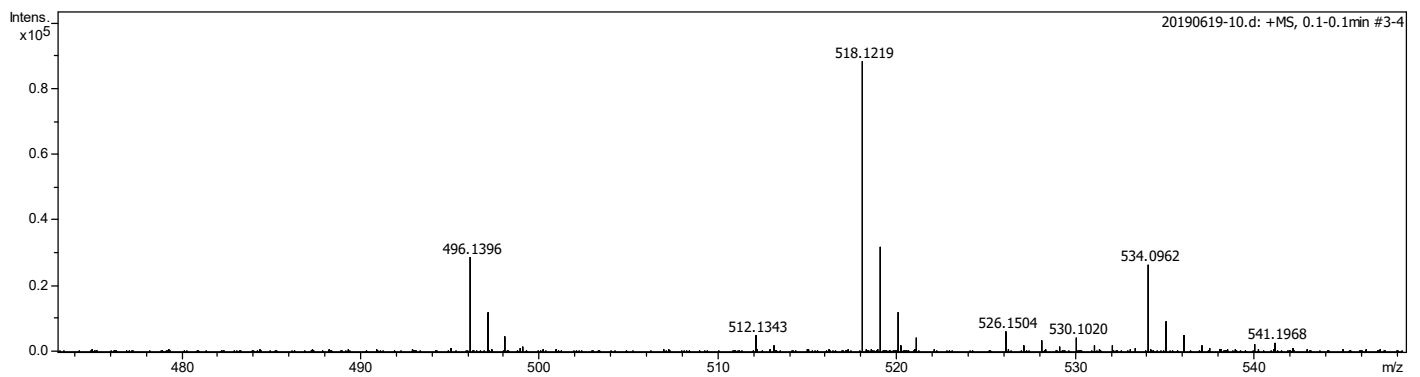

3,4-Bis((4-chlorophenyl)thio)-2,5-diphenyl-1H-pyrrole (3d) [known compounds]

3,4-Bis((4-nitrophenyl)thio)-2,5-diphenyl-1H-pyrrole (3e)

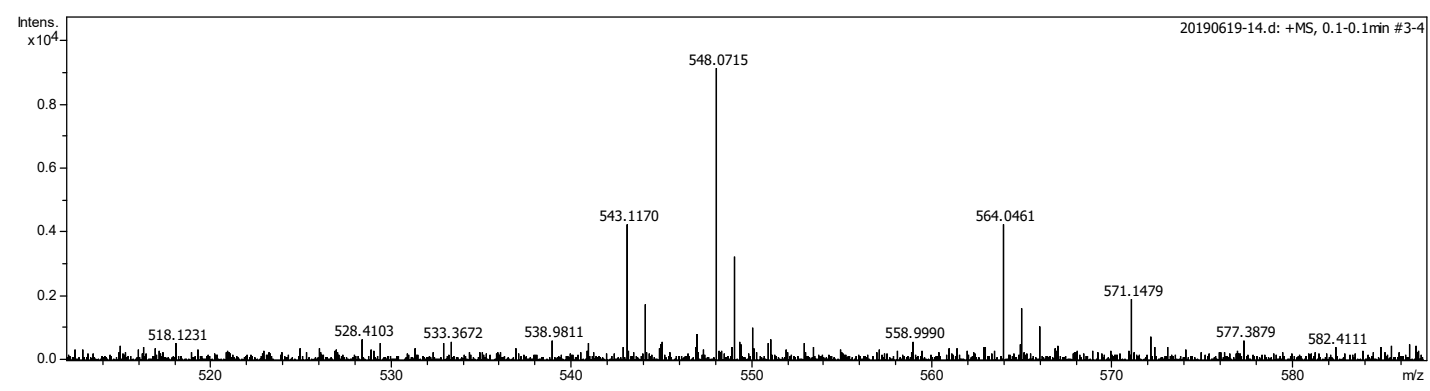

3,4-Bis((4-azidophenyl)thio)-2,5-diphenyl-1H-pyrrole (3f) 


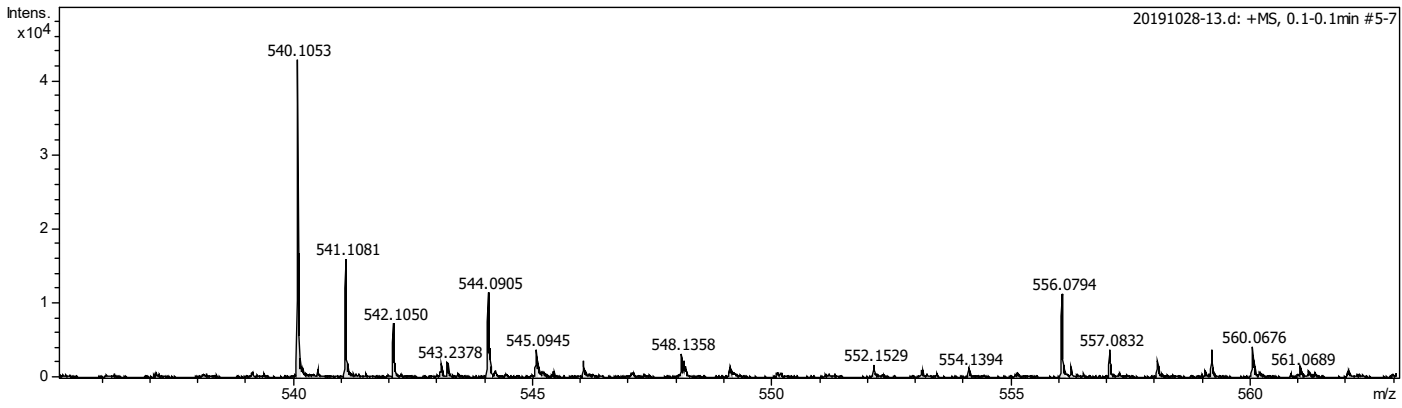

\section{3,4-Bis((2-bromophenyl)thio)-2,5-diphenyl-1H-pyrrole (3g)}

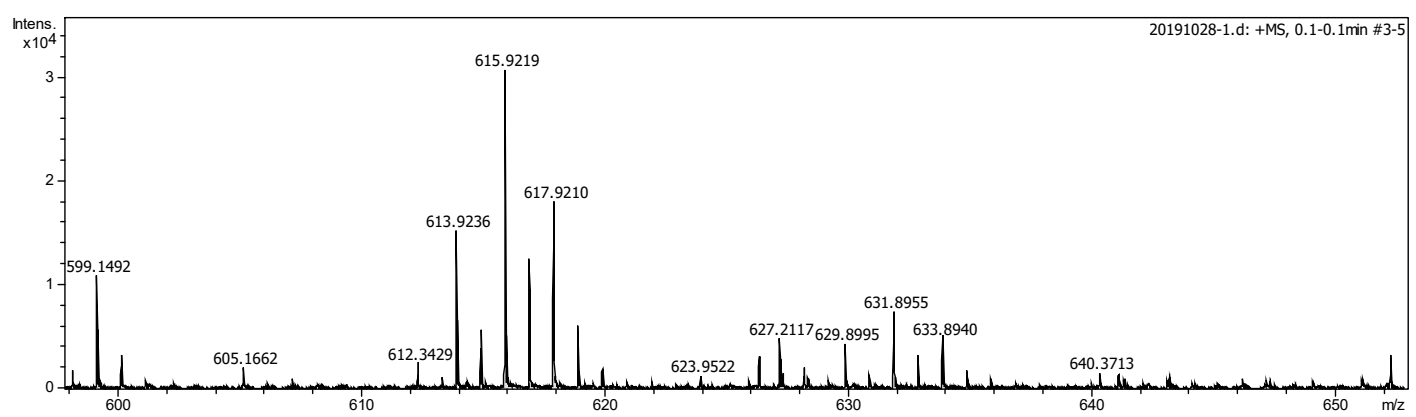

3,4-Bis(naphthalen-2-ylthio)-2,5-diphenyl-1H-pyrrole (3h)

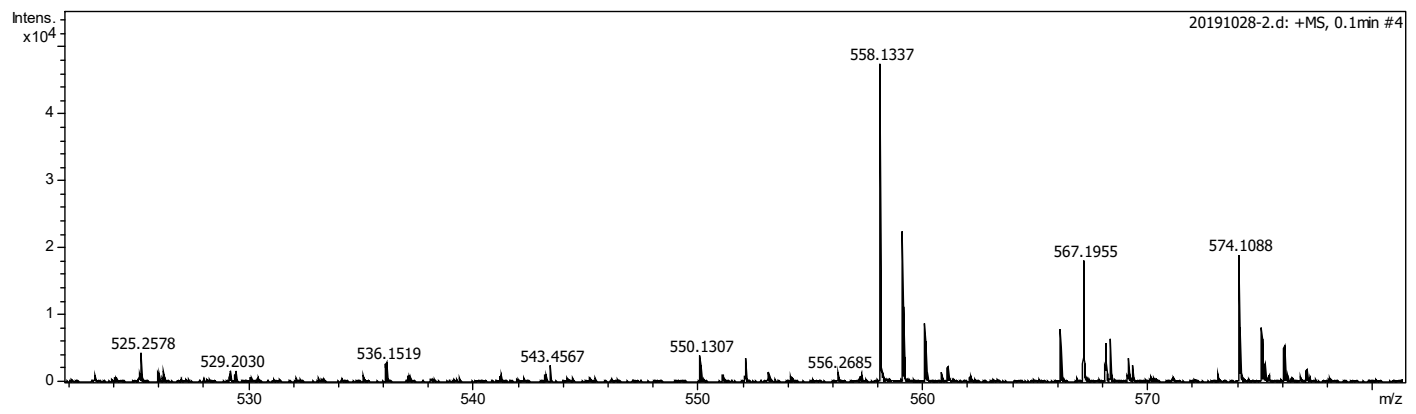

3,4-Bis(methylthio)-2,5-diphenyl-1H-pyrrole (3i)

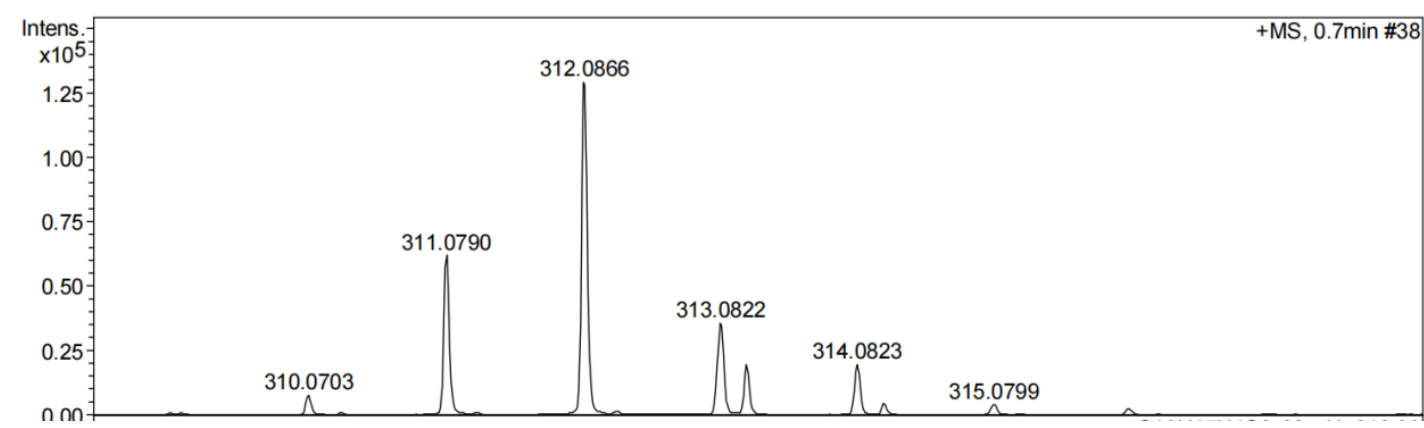

\section{3,4-Bis(ethylthio)-2,5-diphenyl-1H-pyrrole (3j)}

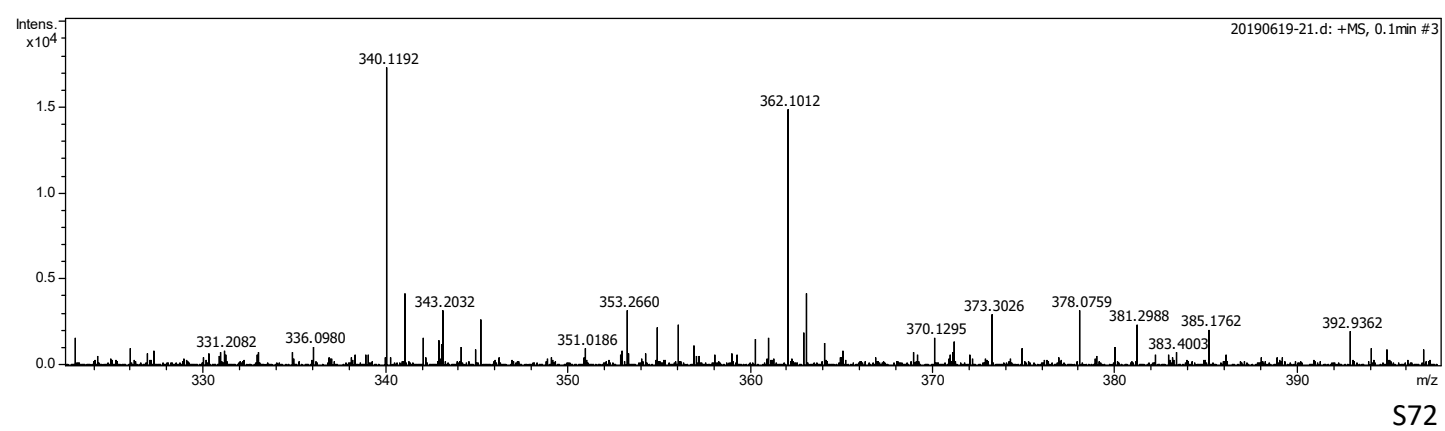


HRMS

3,4-Bis(benzylthio)-2,5-diphenyl-1H-pyrrole (3k)

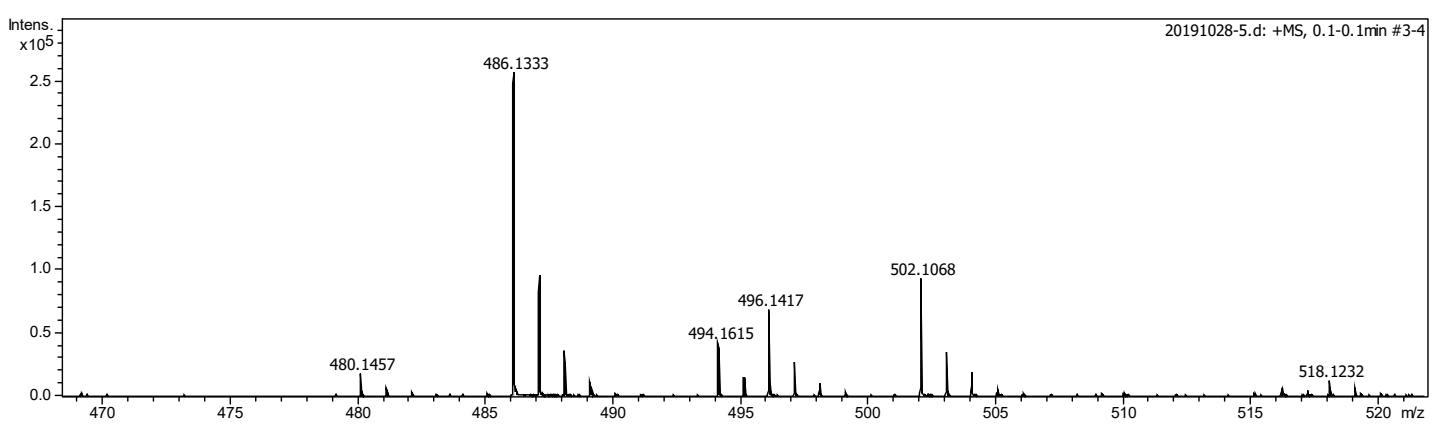

Diethyl 3,3'-((2,5-diphenyl-1H-pyrrole-3,4-diyl)bis(sulfanediyl))dipropionate (3I)

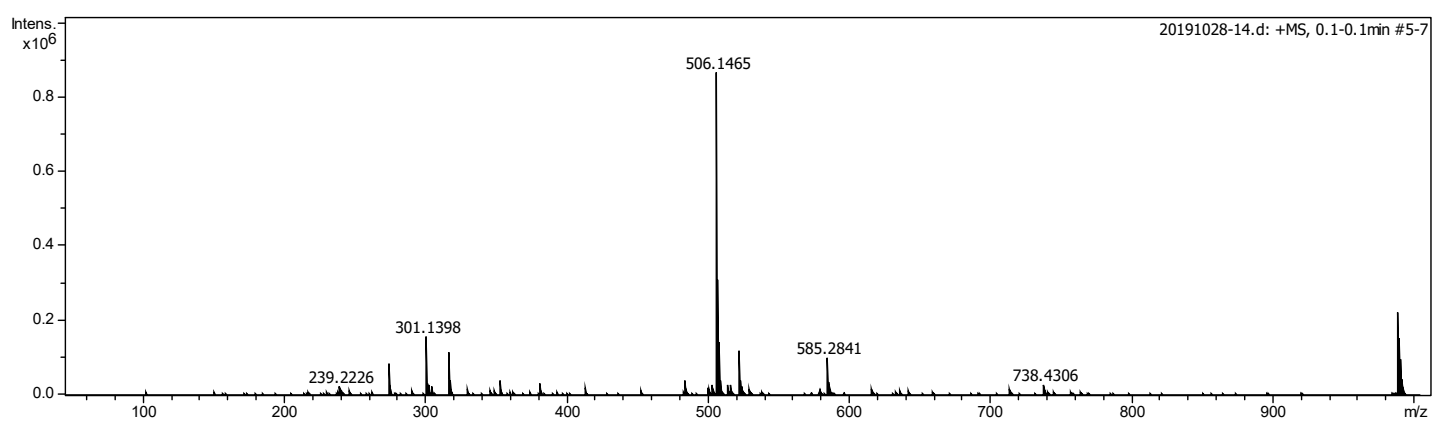

Tert-butyl 3,4-dimercapto-2,5-diphenyl-1H-pyrrole-1-carboxylate (3m)

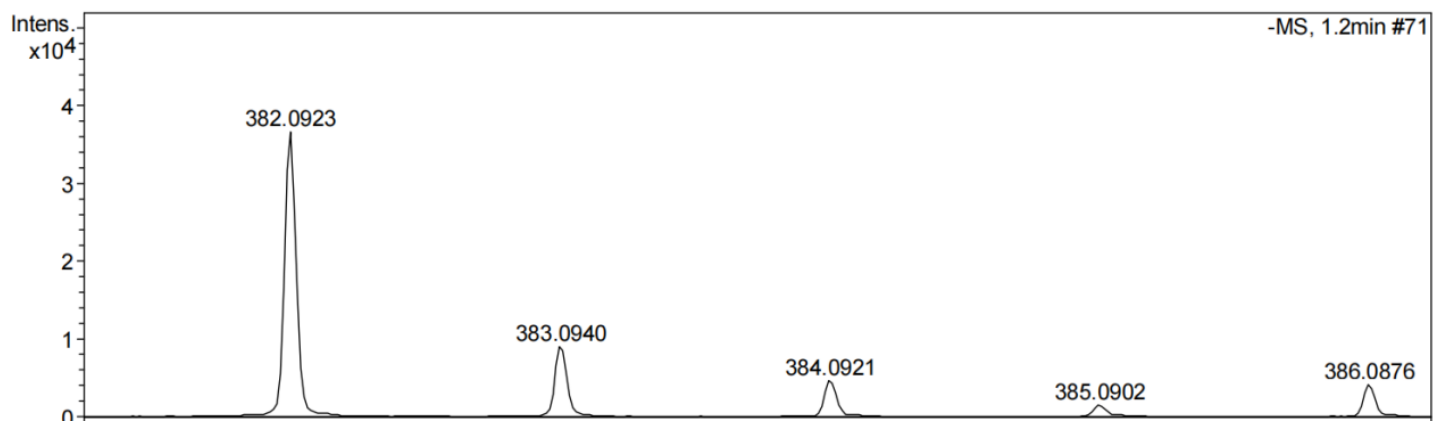

4,6-Diphenyl-5H-[1,3]dithiolo[4,5-c]pyrrole (3n)

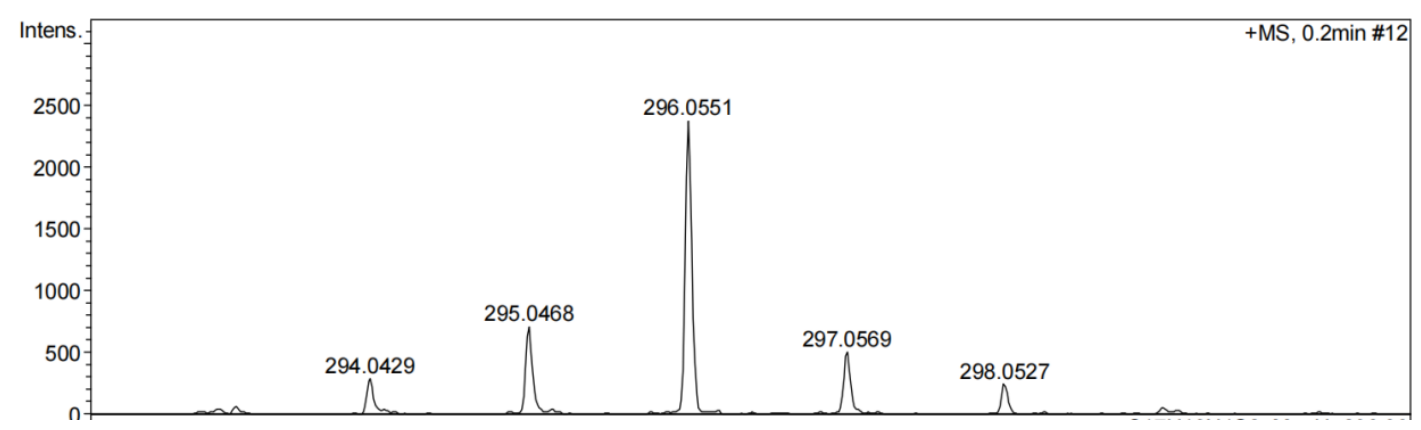

2-Phenyl-5-(p-tolyl)-3,4-bis(p-tolylthio)-1H-pyrrole (3o)

S73 


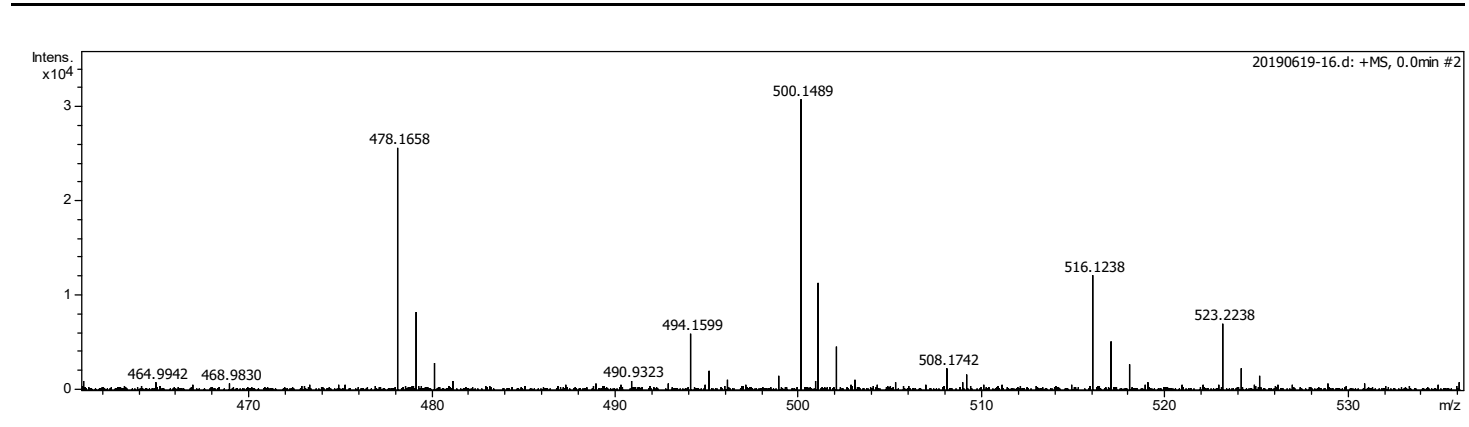

2-(4-Methoxyphenyl)-5-phenyl-3,4-bis(p-tolylthio)-1H-pyrrole (3p)

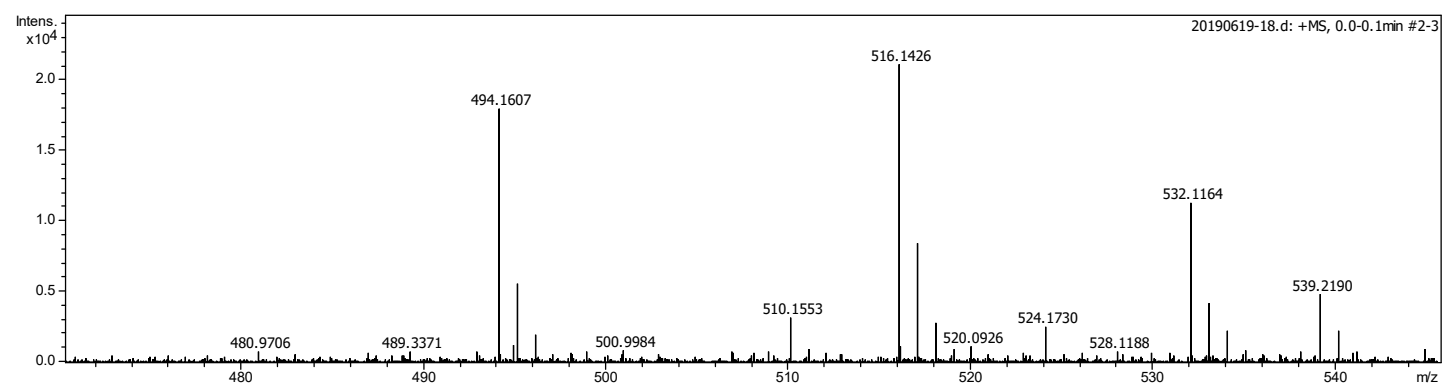

2-(4-Chlorophenyl)-5-phenyl-3,4-bis(p-tolylthio)-1H-pyrrole (3q)

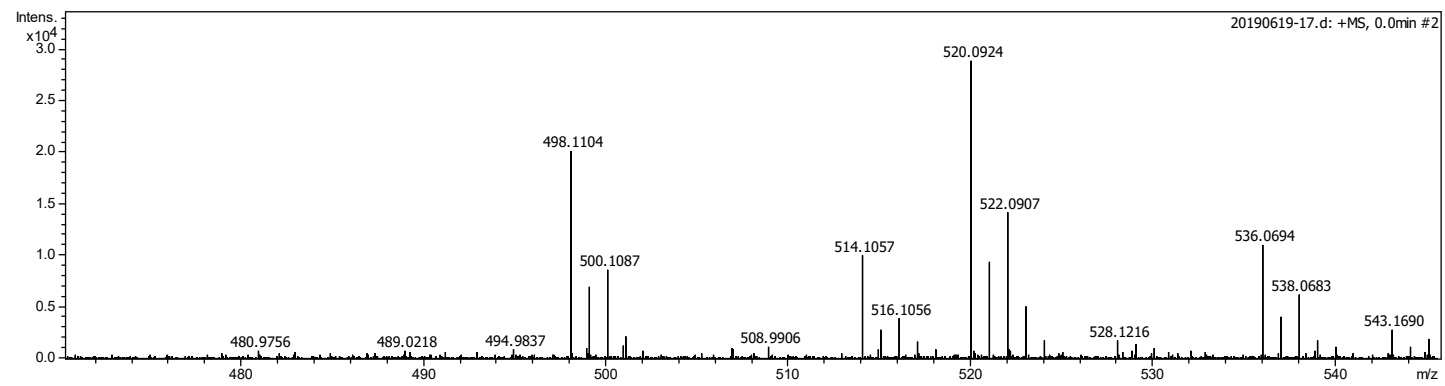

2-Phenyl-3,4-bis(p-tolylthio)-5-(4-(trifluoromethyl)phenyl)-1H-pyrrole (3r)

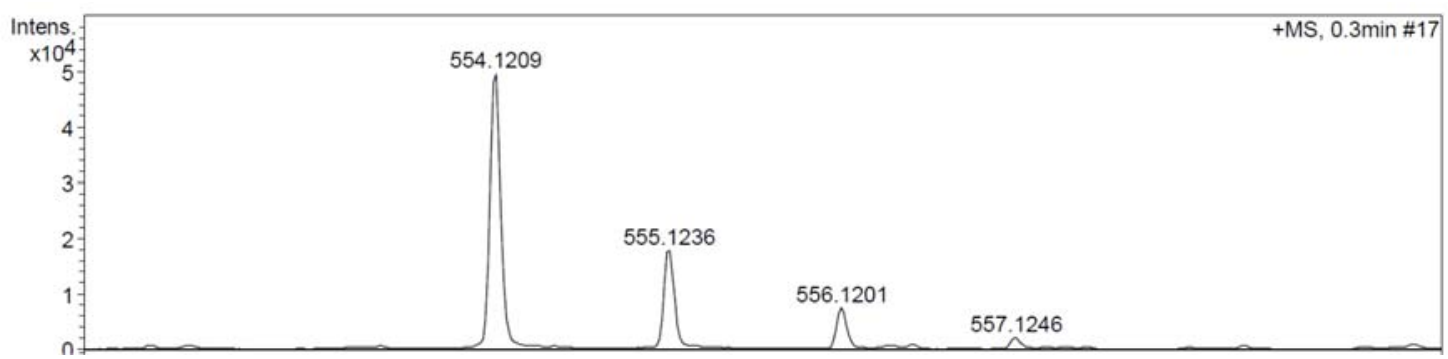

2-Phenyl-5-(thiophen-3-yl)-3,4-bis(p-tolylthio)-1H-pyrrole (3s)

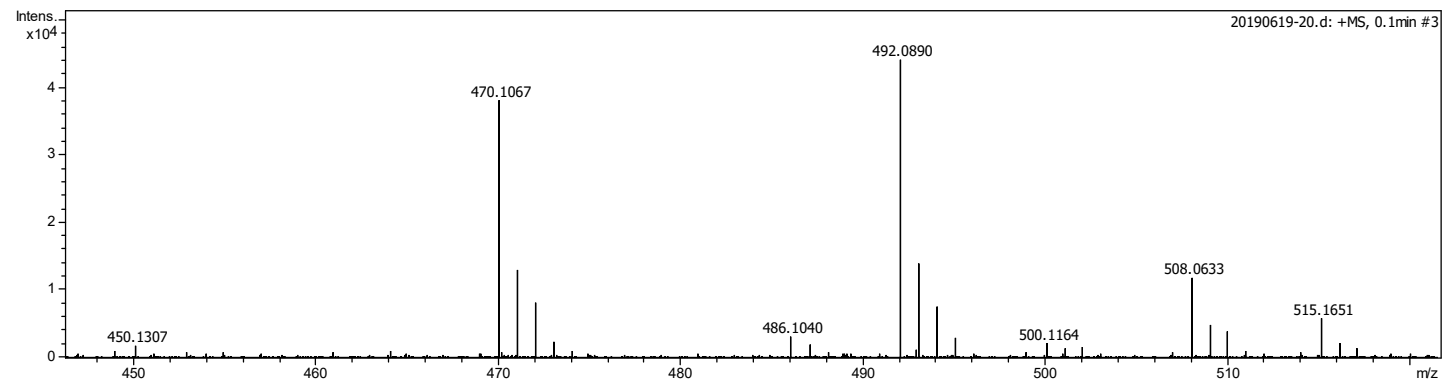

2-Phenyl-5-(thiophen-2-yl)-3,4-bis(p-tolylthio)-1H-pyrrole (3t) 


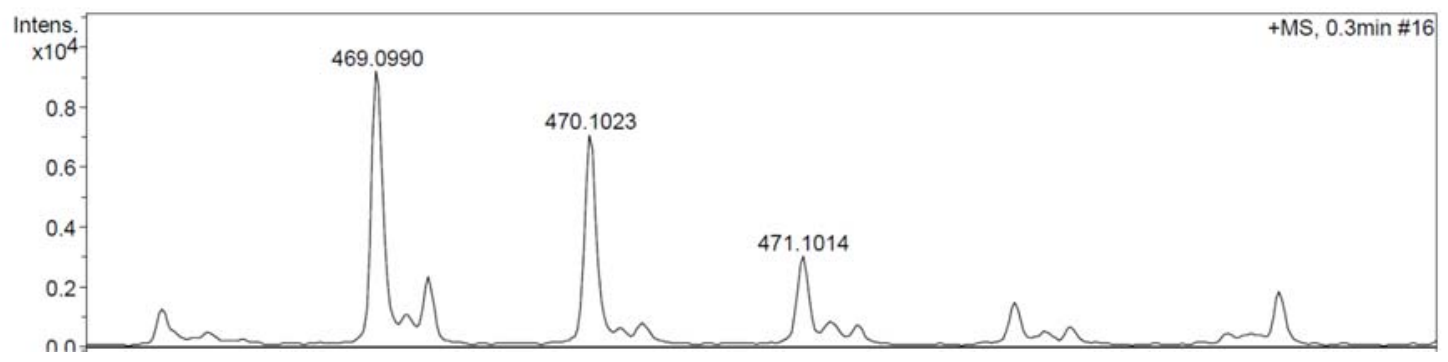

2-Butyl-5-phenyl-3,4-bis(p-tolylthio)-1H-pyrrole (3u)

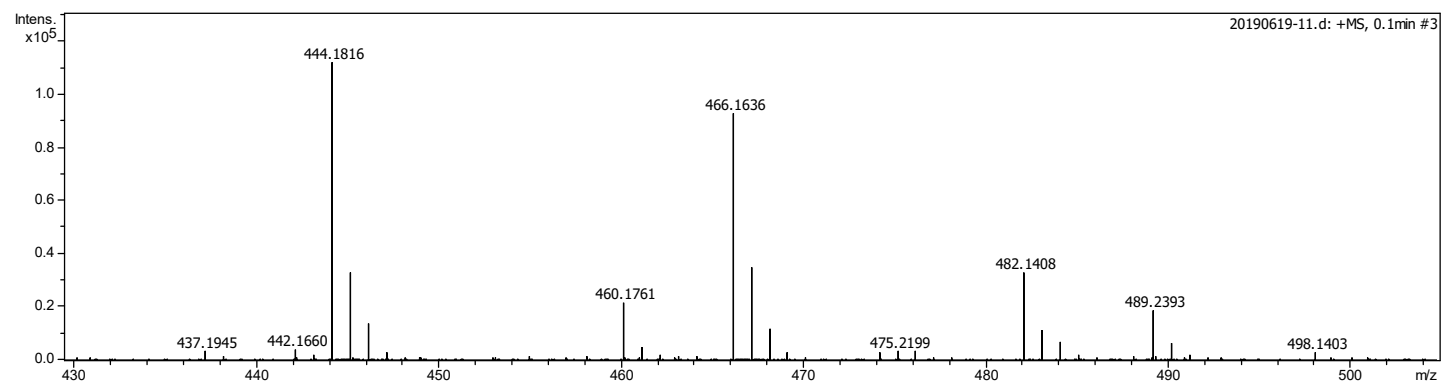

2-Cyclopropyl-5-phenyl-3,4-bis(p-tolylthio)-1H-pyrrole (3v)

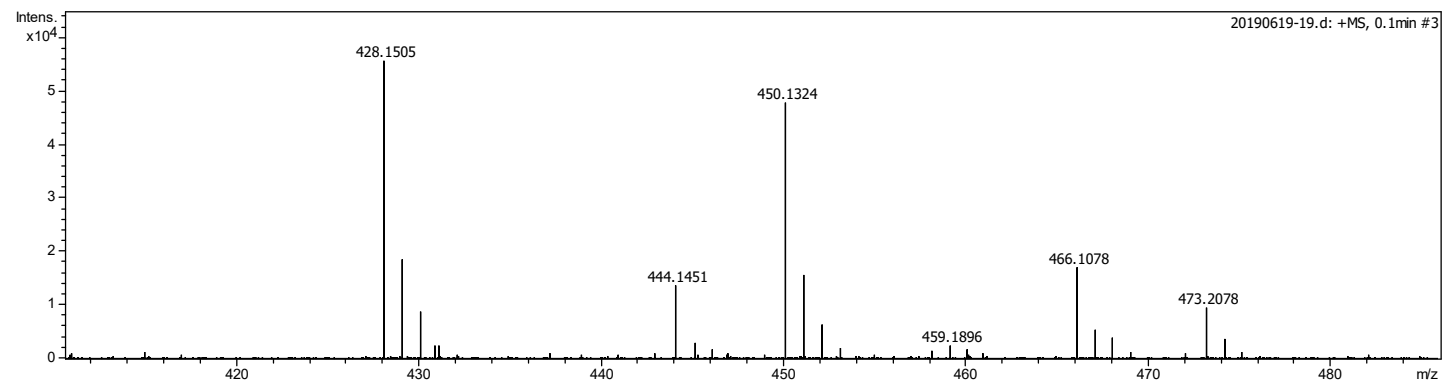

2-Methyl-5-phenyl-3,4-bis(p-tolylthio)-1H-pyrrole (3w)

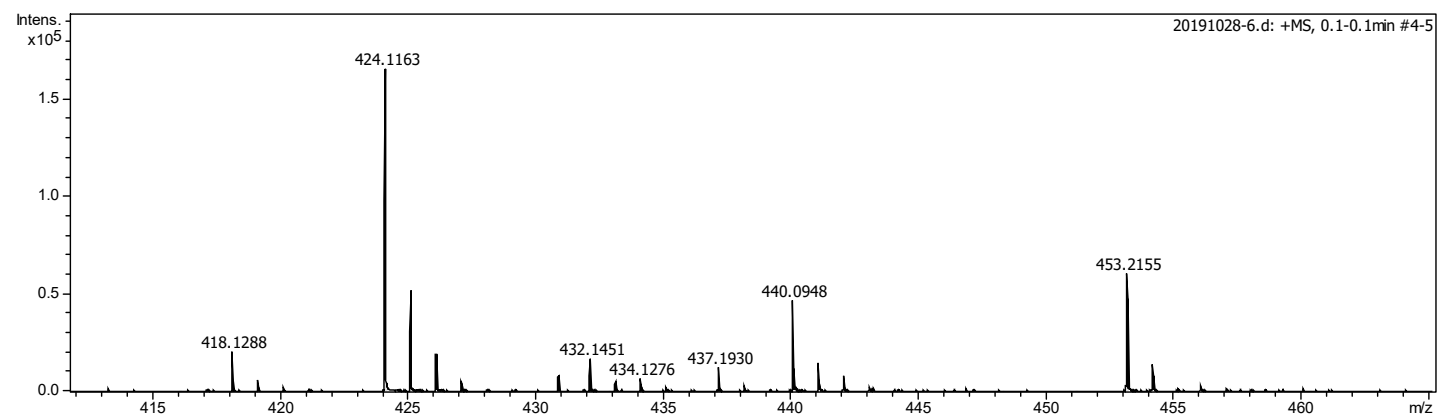

5,7-Diphenyl-2,3-dihydro-6H-[1,4]dithiino[2,3-c]pyrrole (3x)

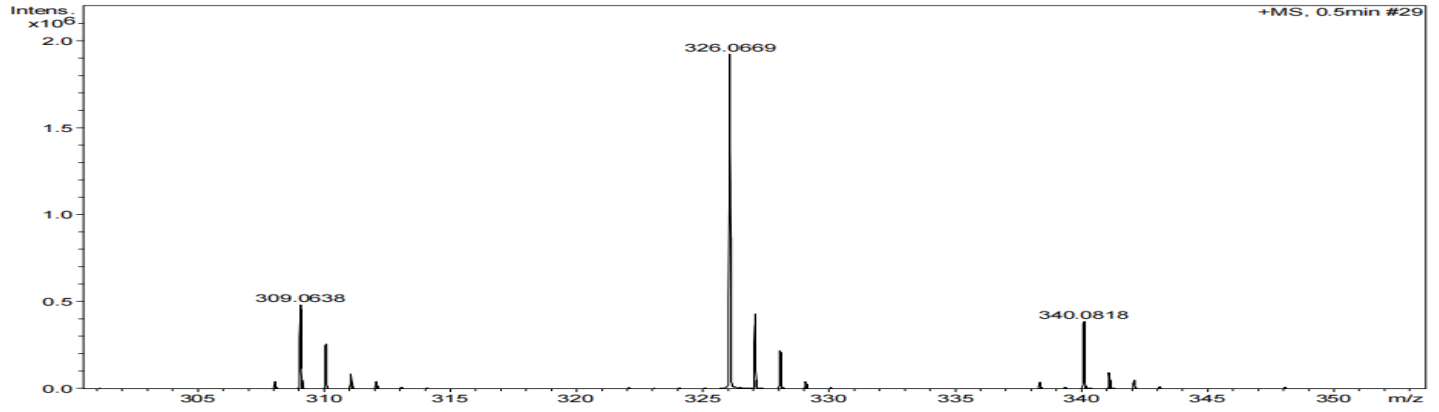

2-Phenyl-3-(p-tolylthio)-4,5,6,7-tetrahydro-1H-indole (3y) 


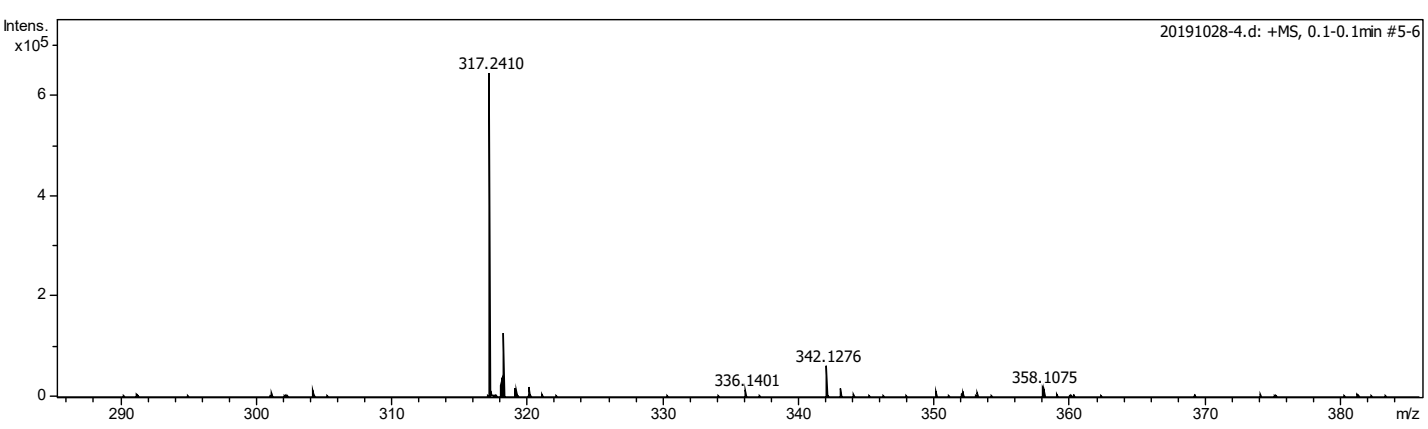

2-Phenyl-3,4,5-tris(p-tolylthio)-1H-pyrrole (3z)

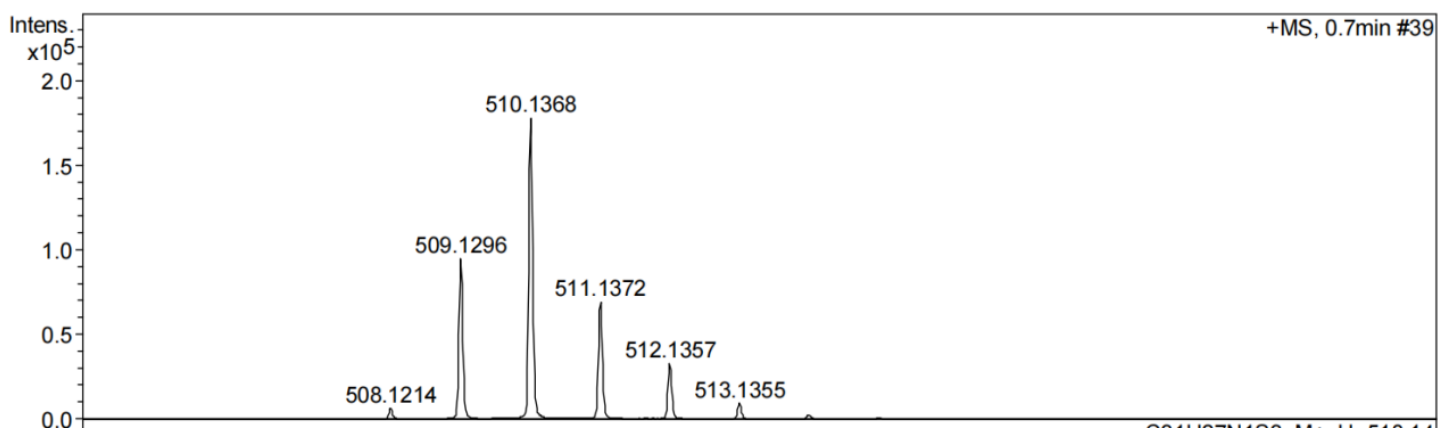

